submitted to Molecular BioSystems

\title{
Functional aqueous droplet networks
}

Michael J. Booth, Vanessa Restrepo Schild, Florence Downs and Hagan Bayley

Department of Chemistry, University of Oxford, 12 Mansfield Road, Oxford, OX1 3TA, UK 
Title/Authors

Index

Abstract

1. Introduction

1.1 Droplet interface bilayers

1.2 Physical properties of DIBs

2. Formation of droplet pairs and networks

2.1 Formation of droplet pairs

2.2 Formation of 2D droplet networks

2.3 Formation of small 3D droplet networks

2.4 Formation of large 3D droplet networks

2.5 Droplet networks in an aqueous environment

3. Functional properties of droplet pairs and networks

3.1 Functional properties of droplet pairs

3.1.1 Useful manipulations of droplets

3.1.2 Sensing and screening

3.1.2.1 Mechanosensors

3.1.2.2 Translocation assays

3.1.2.3 Chips for screening membrane proteins

3.1.2.3.1 Sequential screening

3.1.2.3.2 Parallel recording

3.1.2.3.3 The ideal screening device

3.1.3 Chemical reactions in compartments

3.1.3.1 Conventional chemistry

3.1.3.2 Oscillating reactions and chemical waves

3.1.4 DHB technology

20-21

21

21-22

\section{Future prospects}

3.2 Functional properties of 2D droplet networks

3.2.1 Electronic devices from 2D droplet networks

3.2.2 Power generation in 2D droplet networks

3.2.3 Logic operations in 2D droplet networks

3.2.4 Chemical reactions in 2D droplet networks

3.3 Functional properties of 3D droplet networks

3.3.1 Functional properties of small 3D droplet networks

3.3.2 Functional properties of large 3D droplet networks

3.3.2.1 Electrical signaling in 3D droplet networks

3.3.2.2 Folding 3D droplet networks

3.3.2.3 Controlling 3D droplet networks

3.4 Functional properties of droplet networks in an aqueous environment

3.4.1 Interactions of multisomes with the surrounding aqueous environment

3.4.2 Chemistry and biochemistry in multisomes

3.4.3 Communication and interfaces with other structures and tissues

Abbreviations

References 


\begin{abstract}
Droplet interface bilayers (DIBs), comprising individual lipid bilayers between pairs of aqueous droplets in an oil, are proving to be a useful tool for studying membrane proteins. Recently, attention has turned to the elaboration of networks of aqueous droplets, connected through functionalized interface bilayers, with collective properties unachievable in droplet pairs. Small 2D collections of droplets have been formed into soft biodevices, which can act as electronic components, light-sensors and batteries. A substantial breakthrough has been the development of a droplet printer, which can create patterned 3D droplet networks of hundreds to thousands of connected droplets. The 3D networks can change shape, or carry electrical signals through defined pathways, or express proteins in response to patterned illumination. We envisage using functional 3D droplet networks as autonomous synthetic tissues or coupling them with cells to repair or enhance the properties of living tissues.
\end{abstract}




\section{Introduction}

Here, we describe the properties of designed networks of aqueous droplets. The droplets have volumes in the range of $\mathrm{fL}$ to $\mu \mathrm{L}$ and they are separated from each other by single functionalized lipid-bilayer membranes. As a consequence, the networks can express interesting emergent properties, which cannot be attained by individual compartments. There is therefore an analogy between cells and tissues and individual droplets and networks of them. We have attempted a comprehensive review, highlighting advances, which based on our experience we believe to be sound. When necessary we provide critical comments or flag gaps in our knowledge.

\subsection{Droplet interface bilayers}

Work on droplet networks quickly emerged from our efforts to make miniaturized bilayer systems from droplet pairs for electrical recording from membrane channels and pores. Following a suggestion of David Needham in $2005^{1,2}$, we incubated aqueous droplets of $\sim 200 \mathrm{~nL}$ in an "oil" (e.g. hexadecane) containing a lipid (e.g. a phosphatidylcholine). The droplets became encapsulated in lipid monolayers, and, when droplets were brought together in pairs, a kinetically stable lipid bilayer (a droplet-interface bilayer: DIB) formed at the interface between them (Figure 1A, B). When the droplets were equipped with electrodes, single-channel currents could be recorded after a protein channel or pore contained in one of the droplets entered the bilayer $^{1,3}$. This approach allowed the quantities of a protein under investigation or the amounts of expensive reagents used to be greatly reduced ${ }^{1,3}$. We subsequently discovered that interface bilayers had been made on a macroscopic scale by Tsofina and co-workers ${ }^{4}$, but not further pursued. The groups of Takeuchi $^{5}$ and Schmidt $^{6}$ also began work on similar systems at around the same time as our group. Soon afterwards, the Wallace group explored droplet hydrogel bilayers (DHB) in which a single droplet in oil is interfaced with a lipid monolayer on a hydrogel substrate ${ }^{7}$ (Figure 1C, D). Early work on simple DIBs and DHBs has been reviewed extensively ${ }^{1,2,8-12}$, and is summarized here along with a more extensive discussion of recent developments.

\subsection{Physical properties of DIBs}

The properties of individual droplet pairs are of course pertinent to droplet networks. During the preparation of this review, it became clear that work in this area is fragmented and an orderly analysis of the rates of monolayer and bilayer formation and the physicochemical properties and stability of DIBs, with respect to several variables, remains lacking and would benefit from systematic exploration.

DIBs are thermodynamically unstable towards fusion into a single droplet, i.e. they exist in a local energy minimum. Nonetheless, when prepared and manipulated under favorable conditions, their high kinetic stability allows their use in a wide range of experiments and applications. In this regard, they are more versatile than conventional planar lipid bilayers, which are highly 
susceptible to hydrostatic pressure, mechanical shock or electrostatic disturbance. Members of different classes of membrane proteins including channels, pores and transporters, have been incorporated into $\mathrm{DIBs}^{1}$ (and see Section 2.1).

A variety of bilayer-forming lipids (most, we expect e.g., recently, plant lipids ${ }^{13}$ ) can be used to make DIBs ${ }^{1}$, although by no means have all the possibilities been tested and various incidental issues must be managed. For example, to form DIBs from total E. coli lipids, droplets must first be heated to above the gel-fluid transition temperature $\left(\mathrm{T}_{\mathrm{g}}=44^{\circ} \mathrm{C}\right)$ to promote monolayer formation. DIBs can then be formed either above or below $\mathrm{T}_{\mathrm{g}}{ }^{14}$. Allowing individual droplets to incubate in lipid-containing oil is crucial for the formation of a densely packed and well-ordered monolayer ${ }^{3}$. For smaller droplets, less time is required to form monolayers and hence bilayers ${ }^{15}$. Conversely, when using a mixture of diphytanoyl-sn-glycerol-3-phosphocholine (DPhPC) and a lipid with a poly(ethylene glycol) (PEG) headgroup, it was found that prolonged incubation can significantly increase the time required for bilayer formation ${ }^{16}$.

The strength of a lipid bilayer is derived from the hydrophobic effect, headgroup interactions and the van der Waals forces between the phospholipid tail groups ${ }^{17}$. We assume that droplet pairs with DIBs of larger fractional area are more strongly held together. Therefore, the strength of a DIB can be most readily estimated from as the area of the interface or from the droplet-droplet contact angle (Figure 2). The contact angle is strongly dependent on the nature of the lipid used, its concentration, the oil used (typically decane, dodecane, hexadecane, silicone AR20, and mixtures thereof), the internal contents of the droplets ${ }^{16}$ and the temperature. The oil is an important consideration, because it is often manipulated to control the density or viscosity of the medium, but this can have other consequences. Components of the oil that are poor solvents for the lipid strengthen the bilayer ${ }^{18-21}$. DIB formation by a block copolymer was also highly dependent on the nature of the $\mathrm{oil}^{22}$. The dependence of droplet adhesion on the headgroups and fatty acyl chains of a phospholipid ${ }^{23}$ and the inclusion of cholesterol ${ }^{21}$ have been investigated. For example, the adhesive force was very weak when the fatty acyl chain length was decreased to 14 carbon atoms ${ }^{23}$. The ability to dissociate DIBs, and presumably cleanly separate the two leaflets of the bilayer, either mechanically ${ }^{3,24}$ or by other means, such as dielectrophoresis ${ }^{25}$, is a maneuver that distinguishes DIBs from other bilayer systems, and which has proved useful in several applications, including network remodeling ${ }^{3}$, screening technology ${ }^{24}$ and translocation assays ${ }^{26}$. 'Strong and stable' have been used rather loosely to describe DIBs. The strength of a bilayer should be distinguished from its stability, i.e. it is possible to have a long-lived droplet pair or network from which droplets are readily separated. Pairs or networks in which a fraction of the lipids have pegylated headgroups are an example of stable structures with relatively weak droplet-droplet interactions ${ }^{16}$.

Water loss and droplet shrinkage is an important issue for DIB integrity and the oil surrounding DIB systems must be kept fully hydrated by providing a high humidity environment. Dehydration of 50-500 fL droplet pairs, with lipid in the external oil, produced interesting buckling and eventually blebbing of the 
$D^{27}{ }^{27,28}$ (Figure 3). The nature of the phenomenon differed when DIBs were formed by the lipid-in approach (see Section 2.1); droplet pairs with lipid inside the droplets shrank uniformly. Air-stable DIBs on oil films ${ }^{29}$ are also subject to the effects of dehydration, which has been attributed to water removal from lipid head-groups ${ }^{30}$.

The area of a DIB can be controlled by various manipulations. As we have noted, the strength of a DIB, and hence its area, depends strongly on variables such as the lipid used, its concentration, the oil, the droplet contents and the temperature. Once formed, the area of a DIB can be altered mechanically (Figure 4), e.g. by pulling on an inserted electrode ${ }^{21,31,32}$ (Figure $^{3}$ $4 \mathrm{~A}$ ) or by using a deformable poly(dimethyl siloxane) (PDMS) container $^{33,34}$ (Figure 4B). DIBs can also be manipulated by applying a voltage with electrodes within the droplets, which produces area changes arising from the variation in surface tension with applied potential (electrowetting) ${ }^{21,32,35}$ (Figure 4C). In certain cases, the changes in area have been reported to be very large, e.g. up to $500 \%(\sim 300 \%$ at $100 \mathrm{mV})$ for DPhPC in dodecane ${ }^{36}$ and up to $400 \%$ for DIBs formed with a block copolymer, depending on the nature of the $\mathrm{oil}^{22}$ (Figure 4C). DIB area has an initial linear dependence on the square of the applied potential ${ }^{21,22,32,36}$.

In droplet hydrogel bilayers (DHB, Figure 1C, D), a lipid-coated droplet in oil contacts a lipid monolayer on a flat hydrogel surface. DHBs offer the ability to simultaneously record electrical current and fluorescence (by total internal reflection) ${ }^{11,37,38}$. The formation and stability of DHBs is also poorly understood. There are then many loose ends associated with our understanding of the physicochemical properties of DIBs, and DHBs, and further exploration of this area is encouraged.

\section{Formation of droplet pairs and networks}

\subsection{Formation of droplet pairs}

When DIBs are formed, the lipid can either be dissolved in the oil (lipid-out) or incorporated within the droplets in the form of vesicles (lipid-in) ${ }^{39}$ (Figure 5). Venkatesan and colleagues have shown that the mechanism and rate of monolayer formation differs between the two cases ${ }^{40}$. Monolayer formation was 5 to 10 times faster when using the lipid-in technique. However, we note that a systematic evaluation of the rates of formation of monolayers by different techniques, and with different lipids, oils and droplet contents is needed. "DIBs" have been made by using a triblock copolymer (poly(ethylene oxide)-b-poly(dimethyl siloxane)-b-poly(ethylene oxide)) in place of lipid ${ }^{22}$. Interestingly, in this case, an applied potential was required to form a DIB by an electrocompression process. The DIB reverted into two individual droplets upon removal of the potential. Especially striking is the ability to make asymmetric DIBs (with different lipids in each leaflet) by using two lipid-in droplets, each containing a different lipid mixture ${ }^{13,39}$ (Figure 5B). This is an important manipulation, because most if not all biological membranes are asymmetric with respect to lipid ${ }^{41}$. 
Droplets need not be fully submerged in oil for DIB formation to occur. Boreyko and colleagues formed "air-stable" DIBs, from aqueous droplets containing DPhPC liposomes, on oil films locked into a nanopillared superhydrophobic substrate $^{29}$ (Figure 6). A thin film of oil from the surface migrated around each droplet, allowing a lipid monolayer to form in the film from the internal liposomes. Bringing two droplets together formed a DIB, which could be electrically interrogated. A limitation of this technique was the short lifetime of the DIBs, owing due to droplet evaporation. A lifetime of $4 \mathrm{~h}$ was achieved by decreasing the ambient temperature to the dew point ${ }^{30}$.

The remarkable ability to dissociate DIBs, mechanically ${ }^{34}$ or by other means, e.g. dielectrophoresis ${ }^{25}$ or shearing in a microfluidic device ${ }^{42}$, has been used for various manipulations including "surgery" (droplet removal and replacement) on droplet networks ${ }^{3}$, the screening of channel protein inhibitors $^{24}$ and the assay of translocated materials ${ }^{26,43}$ (see Section 3.1.2). For example, DIBs can be separated simply by pulling on inserted electrodes and then reformed by bringing the original droplets or a new pair together ${ }^{3}$ (Figure 7A). DIBs can also be manipulated by applying potentials across electrodes within the droplets ${ }^{22,32}$ or by external fields. For example, weakly associated droplets dissociated and very weakly associated droplets fused in an external alternating electric field of $5 \mathrm{kV} \mathrm{cm}^{-1}(10 \mathrm{kHz})^{19}$ (Figure 7B).

DIBs can be further stabilized by several means. Placing a plastic partition with an aperture or apertures between the droplets protects the system against mechanical shock and provides a fixed bilayer area. This procedure has proved useful in several circumstances ${ }^{44-48}$. The increased stability allows the manipulation of reagent concentrations on either side of the bilayer by the fusion of donor droplets ${ }^{44,48,49}$ or perfusion ${ }^{44,45,50}$ (Figure 8A). Perfusion of one droplet (of a pair) at the end of a glass capillary has also been achieved ${ }^{49}$ (Figure 8B). Sarles and Leo stabilized DIBs by placing the droplets in a micromachined acrylic "box" with integrated electrodes ${ }^{51}$. Stabilization can also be attained by forming bilayers between two lipid-encased hydrogel objects $^{34}$. Polymerization of poly(ethylene glycol) dimethacrylate to form the gel is carried out either before or after DIB formation. Hydrogel shapes separated by DIBs were also used as building blocks for droplet pairs and networks ${ }^{52}$ (Figure 9A). A lipid-coated hydrogel droplet has been used as onehalf of a bilayer system ${ }^{26,53}$ and as an external electrode for a droplet pair ${ }^{54}$. DIBs have also been stabilized by forming an external organogel from hexadecane and poly[styrene-b-(ethylene-co-butylene)-b-styrene] (SEBS) triblock copolymer. A DIB is formed in the molten gel at $50^{\circ} \mathrm{C}$, which is then cooled slowly to room temperature ${ }^{55}$ (Figure 9B). The use of block copolymers to form the DIB itself can also produce a strengthened system ${ }^{22}$. Recently, droplet networks stabilized within hydrogel capsules have been explored ${ }^{56,57}$ (see Section 2.5). Droplets for the later formation of DIBs have also been stored in a frozen format ${ }^{58}$. While a great deal of work has already gone into the stabilization of droplet pairs and networks, more versatile, if possible general, approaches must still be pursued.

Various means have been explored to move droplets around to form droplet pairs (or higher order structures). Microfluidics approaches were first explored 
by Bai and colleagues who trapped 'AB' droplet pairs (Figure 10A), each of the two droplets differing in content, and observed fluorescein or $\mathrm{H}_{2} \mathrm{O}_{2}$ diffusion across the DIBs ${ }^{59}$. Similar observations were made by Stanley and colleagues with extended $A B A B . .$. constructs formed in a microfluidic device ${ }^{60}$. Additional examples include the use of microfluidics to make asymmetric bilayers ${ }^{61}$, to form and break DIBs ${ }^{42}$, to organize droplets on "rails"62 and to make pairs of very small droplets $(\sim 1.5 \mathrm{pL})^{63}$. Teflon microfluidic tubing has been used to create DIBs within an oil droplet in a bulk fluorocarbon ${ }^{64}$. A major advantage of microfluidics is the ability to generate thousands of replicate structures, rapidly at typical rates of $10 \mathrm{~s}^{-1}$. Additional means to transport droplets for DIB formation include dielectrophoresis ${ }^{25}$ (Figure 10B) and EWOD (electrowetting on dielectric) ${ }^{65}$. The former approach was also used to separate droplet pairs. Droplets have also been manipulated by lasers by using ill-defined thermal effects to drive formation and dissociation ${ }^{66}$. More recently, droplets of $<15 \mathrm{pL}$ volume were brought together by using a single laser-beam optical trap ${ }^{67}$. Droplets containing paramagnetic beads have been manipulated with a magnetic probe ${ }^{68}$.

Members of various classes of membrane protein (channels, pores and transporters) have been incorporated into DIBs (Figure 11), usually from a solution containing a purified protein in a low concentration of detergent, which becomes diluted to well below the critical micelle concentration (CMC) during the incorporation process ${ }^{1}$. Bacterial or viral channels and pores have been most often examined. These are often robust proteins, which can be characterized by single-channel electrical recording for which DIBs are especially useful, and are generally the same proteins that are readily incorporated into conventional planar lipid bilayers. Nonetheless, various eukaryotic ion channels have been introduced into the DHB format from membrane fragments, which were first added to the hydrogel surface followed by lipid-containing oil. The monolayer that formed was then contacted with a lipid-bounded droplet suspended on an electrode ${ }^{69}$. Eukaryotic channels have also been incorporated into DIBs from liposomes ${ }^{46}$ and, in the case of toxins, by microinjection into a droplet ${ }^{70}$. Membrane proteins can be integrated directly into DIBs during in vitro transcription and translation (IVTT) within one of the droplets (also see Section 3.3.2.3), as first demonstrated by Syeda and colleagues $^{24}$, and later by others ${ }^{16,71}$. Recently, a bacterial lactose transporter, lacY, has been incorporated by this approach ${ }^{72}$. Care must be taken to stabilize DIBs towards components of the specific transcription/ translation mix that is being used. The presence of liposomes ${ }^{24,73,74}$ may help under some, but not all ${ }^{71}$, circumstances. The inclusion of lipids with PEG headgroups was recently shown to stabilize DIBs ${ }^{16}$.

\subsection{Formation of 2D droplet networks}

From the beginning of our endeavors with DIBs, it was apparent that networks of droplets would provide interesting functionality unobtainable from simple droplet pairs. With regard to small 2D droplet networks, an important first goal was to position droplets in defined geometries, e.g. three droplets in a triangle rather than a line. Further, when more than one class of droplets was used, 
e.g. differing in contents, the ability was sought to obtain defined combinations of droplets arranged in a predetermined pattern.

In our initial studies, droplets formed with a micropipet or syringe were simply moved into place mechanically, e.g. manually by using a micropipet ${ }^{3}$, and this approach remains useful for forming one or a few copies of a simple structure $^{28}$. The placement of the droplets can be facilitated by using a micromachined template, resembling an egg box ${ }^{3}$ (Figure 12A). Similarly, a molded $^{33,34}$ or micromachined ${ }^{51,75}$ container can be used to hold droplets. Further, a deformable molded container can be used to manipulate the contact area between droplets ${ }^{33,34}$. Small 2D networks have been made from hydrogel shapes $^{52}$ (Figure 12B) by mechanical manipulation. By pulling on inserted electrodes, droplets can be surgically removed from a network and replaced ${ }^{3}$ (Figure 12A). In droplet pairs, the strength and stability of a DIB are affected by the lipid used, the oil composition, droplet content, droplet size, temperature, membrane potential, etc. (see Section 1.2) and these considerations apply to small droplet networks, although they have been hardly examined in this context. Water loss by dehydration is again important. During dehydration, buckling and fission of DIBs occurs when small droplet networks are made by the lipid-out technique, while uniform shrinking occurs when the lipid-in approach is taken ${ }^{28}$ (Figure 3).

Droplets $(400 \mathrm{~nL})$ containing paramagnetic beads have been manipulated with a magnetic probe, which is brought into proximity with a droplet but does not contact it directly ${ }^{68}$ (Figure 13A). In addition (see Section 2.3), bead-free droplets can be moved by using a bead-containing droplet as a carrier to which a droplet of interest or a short chain of droplets (a "construction module") is attached by DIB formation. After patterning, the magnet is removed by a sharp vertical movement ${ }^{68}$. Friddin and colleagues have used optical tweezers to manipulate aqueous droplets of $<30 \mu \mathrm{m}$ diameter (14 pL) to form small networks connected through DIBs ${ }^{67}$. Additional means to transport droplets include dielectrophoresis ${ }^{25}$ and EWOD (electrowetting on dielectric) ${ }^{65}$ (see Section 2.1). Acoustic waves have been used to organize particles, including cells ${ }^{76}$, and might be applied to aqueous droplets.

Microfluidics have been used to prepare small droplet networks, notably by the groups of Ces, Morgan, Cooper and Garsecki. Earlier studies had demonstrated the formation of water-in-oil-in-water $(\mathrm{w} / \mathrm{o} / \mathrm{w})$ droplets in which oil drops contained a predetermined number of water droplets stabilized by surfactants ${ }^{77,78}$. Stanley and colleagues used a microfluidic system to fill small chambers with lipid-in droplets (5 to $100 \mathrm{~nL}$ ), which formed DIBs to produce long rows of connected droplets ${ }^{60}$. Rows of droplets with alternating contents could be formed by this and similar processes $28,79,80$. The process was improved by Elani and colleagues who also made long rows of droplets (14 to $180 \mathrm{pL}$ ) connected by DIBs, as well as double, triple and quadruple rows ${ }^{61}$ (Figure 13B, 14A). The triple and quadruple rows were 3-dimensional (see Section 2.3) (Figure 14A). The single and double rows could display repeating patterns of two or three types of droplet (Figure 13B). This was possible in the double rows because the droplets emerged from the microfluidic system in zigzag fashion ${ }^{61}$. King and colleagues generated large droplets $(0.7$ to $6 \mu \mathrm{L})$ in a 
microfluidic system that produced linear arrays by using a rail ${ }^{81,82}$ to control droplet movement into a pillar trap, including the positioning of droplets with different contents ${ }^{83,84}$. A delay line allowed sufficient time for lipid monolayer formation before droplet contact ${ }^{83,85}$. Osmotic water movement and sequential bilayer lysis by melittin were examined in droplet chains ${ }^{81}$. Importantly, electrical recordings have been made by embedding electrodes in microfluidic systems $^{42}$. Bilayer area can also be altered by controlling the flow rate in a pillar trap ${ }^{42,81}$. The use of rails to generate rows of droplets $(0.2 \mathrm{~nL}$ to $1.5 \mathrm{~nL}$, up to 20 sequential DIBs) was also demonstrated by the Ces group ${ }^{62,85}$, who additionally trapped clusters of up to 5 droplets in wells ${ }^{85}$.

Microfluidics approaches have advantages for the formation of small DIB networks, including speed, the possibility of automation, the ability to replicate networks uniformly, and the means to handle small ( $p L$ ) droplets. However, there are downsides. For example, various simple arrangements of droplets are not readily attained and the ability to pattern droplets with different contents or of different lipid compositions is limited. It is also difficult to access networks within or retrieve networks from most microfluidic devices.

\subsection{Formation of small 3D droplet networks}

There has been surprisingly little work on the fabrication of small 3D droplet networks. A sustained effort was made by Wauer and colleagues, who built objects, including pyramids, of up to 14 droplets $(\sim 400 \mathrm{~nL})^{68}$ (Figure 14B). The droplets were manipulated in various ways. Initially, they were simply taken up into a pipet and positioned by expulsion from the pipet. More interestingly, droplets were filled with paramagnetic beads so that they could be manipulated in oil with a small, powerful Nd magnet on the end of a glass rod, which was moved with a micromanipulator (see Section 2.2). The geometry of the base of a structure could be set by using a micromachined foundation (see Section 2.2).

In these 3D structures, each droplet was placed in a designated position. When two classes of droplet were used, the precise placement produced specific functional properties. For example, the $\alpha \mathrm{HL}$ pore was included in selected droplets in a pyramid, causing specific DIBs in the structure to be permeable to small ions and molecules. The expected conductive pathways within a structure were shown to be present by the measurement of ionic currents and the movement of fluorescent dye molecules ${ }^{68}$.

Additional efforts to build 3D structures have used microfluidics. Droplets can be packed with precise stoichiometries within oil drops ${ }^{78}$, and a similar approach might be applied to lipid-encapsulated droplets. However, when two or more types of droplet are used, their arrangement cannot be fully controlled, which is a critical issue. Similarly, chains of droplets connected by DIBs have been produced by microfluidics ${ }^{61}$ (Figure 13B, 14A). Two types of droplet can be arranged in an alternating pattern in a chain. But, when 3D bundles of up to 4 chains were made, the alignment of the chains could not be controlled. The use of microfluidics also limits the geometry of a network and the accessibility of the component droplets. 
Laser manipulation can be used to build small, designed droplet structures in 3D. Two-dimensional structures of up to 4 droplets $(\sim 30 \mathrm{~nL})$ have been constructed by using IR-laser manipulation, which acts through a combination of thermal influences, including the thermocapillary effect and convective flow $^{66}$. It also possible to produce droplet fusion by disrupting a DIB with the same laser, thereby augmenting the available building tools by selectively increasing compartment volumes and mixing their contents. Recently, droplets $<15 \mathrm{pL}$ in volume were brought together by using a single laser-beam optical $\operatorname{trap}^{67}$ (Figure 14C). The authors assembled a 3D pyramid of eleven identical droplets. The 3D-printing device described in the next section might also be adapted for the fabrication of small constructs.

\subsection{Formation of large 3D droplet networks}

To create 3D aqueous droplet networks with functional properties, the precise placement of numerous droplets in defined geometries and patterns is required, which cannot be accomplished with methods such as microfluidics and magnetic manipulation. To achieve this a 3D printer was developed, which can produce 3D networks containing thousands of aqueous droplets in defined patterns ${ }^{15}$ (Figure 15). A printing nozzle made from a glass capillary $(1 \mathrm{~mm}$, internal diameter) was manually pulled and the end trimmed to an external diameter of $\sim 100 \mu \mathrm{m}$. The glass nozzle was attached to a poly(methyl methacrylate) (PMMA) chamber containing a piezoelectric transducer (piezo). While the PMMA chamber could held up to $500 \mu \mathrm{L}$ of the printing solution, the volume could be reduced to as little as $3 \mu \mathrm{L}$ for valuable samples, by using an oil plug. By using a micromanipulator, the printing nozzle was submerged in a glass or PMMA reservoir containing DPhPC in a 1:1 mixture of hexadecane and silicone AR20 oil. Droplets were then ejected on demand by activation of the piezo. Pulses of up to $30 \mathrm{~V}$ were triggered by a computer through a microcontroller board. By varying the applied voltage and the pulse duration, droplets with diameters between 10 and $200 \mu \mathrm{m}(0.5 \mathrm{pL}-4 \mathrm{~nL})$ were obtained. Lipid monolayers must have formed within 1 second of droplet ejection (see Section 2.1), allowing a reasonable printing rate ( 1 droplet per second). The $3 \mathrm{D}$ printer handled two nozzles simultaneously, allowing the patterning of droplets with two different contents.

Optimization was required to consistently produce large 3D networks with droplets of uniform size ${ }^{15}$. Fluid levels in the PMMA chamber affected the droplet size and uniformity; lower liquid levels meant less coverage of the piezo disk, which then required a larger force to produce the same size droplets. Furthermore, because the print nozzles were produced manually, slight variations in the nozzle shape meant that different voltage and pulse conditions were required for each to produce droplets of a given size. Leakage of the printing solution from the nozzle was also observed due to hydrostatic pressure, because the PMMA chamber was held above the oil level in the container. To combat this, short square waveforms were applied, which did not eject a droplet but instead returned the oil-aqueous interface on the printing nozzle to a planar configuration. 
In parallel with the optimization of droplet ejection, a program was written to automate the printing of large 3D-patterned networks ${ }^{15}$. A graphical user interface (GUI) was interfaced with the program, which positioned the printing nozzle in three dimensions and allowed real-time control of the voltage pulse duration and amplitude. The droplet size was manually adjusted to the desired initial value prior to automated printing. The printing program generated 3D networks layer-by-layer from 2D arrays, each generated by back-and-forth raster deposition. The map of each $2 \mathrm{D}$ layer is read from a png image file, in which each pixel represents a single droplet, and the pixel color represents which print nozzle to eject from. Theoretically, networks of droplet spheres would adopt perfect 3D hexagonal close-packed lattice structures. However, if there is no interstitial oil within the network, as a result of strong DIB formation, each internal droplet would form a trapezo-rhombic dodecahedron, where each side has formed a bilayer with a neighboring droplet. In practice regularity is difficult to obtain throughout the whole structure for several reasons. As two droplets do not form a DIB instantaneously, there are imperfections in the packing caused by droplets rolling down the sides of printed networks, instead of forming stable contacts. Also, the force produced by newly ejected droplets can displace droplets that have not yet formed bilayers. These issues were minimized by reducing the speed of printing with time delays following the ejection of each droplet and by raising the height of the nozzle with respect to the surface of the growing network. Decreasing the rate at which droplets sank, by altering the ratio of hexadecane to silicone oil, also improved droplet network formation, in terms of regularity, patterning and overall shape. The most uniform 3D-printed structures were generated with potassium chloride-containing droplets with a diameter between 50 and 100 $\mu \mathrm{m}$ (65 to $500 \mathrm{pL}$ ) by using DPhPC as the lipid and a $1: 1$ mixture of hexadecane and silicone AR20 oil. With these optimized conditions, a 3D droplet printer with two printing nozzles was used to generate patterned networks from droplets with different contents ${ }^{15}$ (Figure 15). Challita and colleagues have recently built a similar printer from which droplets emerge from a pneumatically driven head and are released by a "snap-off" technique in which the tip of the nozzle is moved through the oil-water interface ${ }^{86}$. Droplets with diameters as low as $100 \mu \mathrm{m}$ can be printed at $\sim 0.5 \mathrm{~s}^{-1}$.

Calculations demonstrated that the upper limit on the height of 3D networks would be in the thousands of droplets ${ }^{15}$. If they were any taller, the weight of the upper droplets would push apart the bilayers of the droplets below, causing the structures to collapse. However, for smaller droplets or those with more adhesive bilayers (see Section 1.2), the limit will be higher although it is likely that networks of $\mathrm{cm}$ dimensions would be incorporated into supporting structures. Theoretical predictions of the Young's modulus of droplet networks (the ratio of the stress applied to a material compared to the resulting strain) gave a value of $100-200 \mathrm{~Pa}$. This is comparable to the moduli of soft tissues such as the brain ${ }^{87}$. Careful mechanical measurements are required to fully evaluate the viscoelastic properties droplet networks. 


\subsection{Droplet networks in an aqueous environment}

A longer-term prospect is to use droplet networks in medical applications where, for example, they might be interfaced with biological tissues to control or enhance cellular behavior. However, droplet networks residing in oil are incompatible with aqueous physiological environments and cannot make functional interfaces with the lipid bilayers of cell membranes. Therefore, there is a pressing need to generate droplet networks in aqueous environments.

In early work, multi-compartment liposomes were generated by using a w/o/w system $^{88}$. Chloroform and diethyl ether were added to a small volume of neutral oil (e.g. a triglyceride) containing an amphipathic lipid. An aqueous phase containing a molecule to be encapsulated, as well as $0.15 \mathrm{M}$ sucrose or a salt, was added to the organic phase, and a water-in-oil emulsion was formed by shaking. This emulsion was added to a second aqueous phase containing $0.2 \mathrm{M}$ sucrose, and shaken once more to form a w/o/w emulsion. Subsequent evaporation of the organic solvents resulted in liposomes with multiple internal compartments, separated from one another and the exterior environment by lipid bilayers. Triglyceride was present at some of the interstices. The structures were stable for at least 9 days. Molecules that could be encapsulated within them included drugs, dyes, enzymes and biomolecules. Deficiencies of this approach include size heterogeneity of the internal compartments and the inability to pattern the compartments.

These deficiencies were surmounted by the fabrication of multisomes, which are small aqueous droplet networks constructed within an oil drop immersed in a bulk aqueous medium ${ }^{89}$ (Figure $16 \mathrm{~A}-\mathrm{C}$ ). Multisomes are aqueous droplet networks that exist in a bulk aqueous medium. These are ordinarily formed in an oil environment, which in some instances can be completely removed. Manually prepared multisomes have been formed as droplet networks within oil drops of $\sim 270 \mathrm{~nL}$ comprising 9:1 (v/v) silicone oil and hexadecane containing DPhPC (0.1-0.2 $\left.\mathrm{mg} \mathrm{mL}^{-1}\right)$ and surrounded by aqueous medium. A lipid monolayer formed at the periphery of the oil drop. Then, $\sim 14 \mathrm{~nL}$ droplets were pipetted into a separate solution of the lipid-containing oil. After $\sim 5$ minutes to allow the formation of an external monolayers, the droplets were transferred by micropipet into the oil drop. Multisomes were formed either by transferring an intact network of aqueous droplets, or by transferring unconnected droplets, which then formed DIBs. External bilayers were formed between the internal aqueous droplets and the aqueous environment. Multisomes with lifetimes of at least $24 \mathrm{~h}$ were prepared in this way ${ }^{89}$. $\alpha \mathrm{HL}$ pores can be incorporated into the internal and external bilayers of manually generated multisomes, allowing the movement of ions and dyes between compartments $^{89}$.

Multisomes can also be formed by transferring pre-formed droplet networks from bulk oil to a bulk aqueous phase by passing the structures through a lipid monolayer at an oil-water interface under gravity ${ }^{90}$ (Figure 16D-G). Networks of a two to six droplets were created in bulk oil containing 1,2-dioleoyl-snglycero-3-phosphocholine (DOPC) above an aqueous phase. The droplets 
formed bilayers both with each other and with the monolayer at the oil-water interface. Then, as they moved into the aqueous phase, the newly formed droplet network became enveloped in a single bilayer. However, this formation process does not provide precise control over the network geometry. Additionally, the densities of the droplets, the bulk oil and the bulk aqueous phase had to be optimized to allow gravity to overcome the tension of the monolayer. Under the conditions tested, each droplet compartment had a minimum volume of $\sim 65 \mathrm{~nL}$, below which the gravitational force acting on the droplets could not overcome the surface tension at the interfacial monolayer. After phase transfer, the high sucrose concentration inside the aqueous compartments of the multisomes, relative to the bulk aqueous phase, resulted in a destabilizing osmotic pressure. To reduce this imbalance, sucrose was injected at the base of the bulk aqueous phase before droplet generation. Two-compartment multisomes had a lifetime of $\sim 60$ minutes and the initial yield was $43 \%$. The yield was improved to $83 \%$ by encapsulating liposomes within the compartments, which stabilized the bilayers. $\alpha \mathrm{HL}$ pores were incorporated into internal and external bilayers by creating droplets from an $\alpha \mathrm{HL}$-containing buffer, or by putting $\alpha \mathrm{HL}$ in the external medium. In later work, aqueous compartments contained an IVTT protein expression system, which was used to produce different fluorescent proteins in situ in different compartments $^{74}$. However, the presence of macromolecules from the IVTT system inside the aqueous compartments disrupted the bilayers, decreasing the yield to $12 \%$. This problem might be remedied by using lipids with PEGmodified headgroups, which have been shown to stabilize DIBs exposed to biological macromolecules ${ }^{16}$.

Multisomes have also been made by a microfluidic approach in which up to four droplets ( 4 pL) were enclosed within an oil drop ${ }^{63}$ (Figure 17A, B). The droplets are far smaller than those assembled by micropipeting in the manual approach to multisomes ${ }^{89}$ and the multisomes were generated at a rate of hundreds per minute. In the microfluidic device, two buffer-containing channels formed a three-way junction with a channel holding a DPhPC-containing oil phase, which allowed aqueous droplets with two different contents to form and acquire stable lipid monolayers. At a second junction, with another aqueous channel, a specific number of aqueous droplets was encapsulated in a larger oil drop, forming a multisome stable for at least 60 minutes. The number of encapsulated droplets could be tuned to between one and four by changing the frequency at which droplets were generated at the first junction.

Oil-free multisomes with homogeneously-sized internal compartments have also been microfluidically generated by controlling the interfacial energies in a w/o/w system ${ }^{91}$ (Figure 17C, D). This was achieved by adding a triblock copolymer surfactant, Pluronic F-68, into the outer aqueous phase, which reduced the interfacial energy between the external water and the oil phase and allowed the oil to separate from each multisome as an individual droplet (dewetting). By manipulating the flow rate in the system, multisomes could be formed with more than thirty unpatterned compartments. However, the yield of 2 -compartment structures was only $62 \%$ due to bilayer instability during the dewetting process. Protein pores were incorporated into the bilayers of 2compartment multisomes, allowing the selective diffusion of dye molecules 
between compartments and between a compartment and the external environment.

By using microfluidics, multisomes can be encapsulated in hydrogel shells to improve their stability ${ }^{56}$ (Figure 17E). Aqueous droplets were first generated in a tube containing lipid-in-oil. Multiple droplets, surrounded by a small volume of oil, were then ejected into a secondary, coaxial tube containing the shell phase, an aqueous alginate solution with suspended $\mathrm{CaCO}_{3}$ particles. Then, each enveloped oil drop (containing several aqueous droplets) was ejected into a third coaxial tube, in which the carrier phase contained mineral oil with dissolved acetic acid. The acid partitioned into the solution-phase alginate shell, causing the liberation of $\mathrm{Ca}^{2+}$ ions and ionic crosslinking of the alginate. Once formed, the encapsulated multisomes were stable for weeks, and could be transferred into air or aqueous environments (Figure 17F).

In a related endeavor, Bayoumi and colleagues manually injected DPhPCcontaining oil drops of $50-200 \mu \mathrm{L}$ into agarose as it gelled. Aqueous droplets of $<50 \mathrm{~nL}$ containing DPhPC liposomes were then similarly injected into the oil drops, where they formed DIBs with each other and bilayers at the wateragarose interface ${ }^{57}$. Assemblies with various droplet numbers and geometries were made, which were stable for more than 3 days. The integrity of the bilayers was confirmed by capacitance measurements, and the ability of the pore-forming protein ClyA to transport ions and dye molecules through them.

By using the 3D droplet printer (see Section 2.4), multisomes comprising thousands of aqueous droplet compartments inside a single oil drop were created $^{15}$ (Figure 18A). Printing was carefully programmed to navigate the curved oil-drop surface geometry. Following printing, excess oil inside the oil drop was removed through suction into the printing nozzle, leaving behind only the aqueous compartments. Like the microfluidically generated multisomes, the 3D-printed multisomes contained pL-sized droplets, as opposed to manually constructed multisomes, which contained-nL-sized droplets. Additionally, the 3D-printed networks were stable for several weeks in water. Importantly, the internal droplets could be patterned (Figure 18B). The inclusion of membrane proteins within these larger structures would allow long-distance communication between internal compartments, and with the external environment.

\section{Functional properties of droplet pairs and networks}

\subsection{Functional properties of droplet pairs}

Droplet pairs were originally investigated as miniaturized bilayer systems for electrical recording from membrane channels and pores ${ }^{1}$. In this section, we discuss droplet pairs, and also "chips" and devices used for screening; most of the latter incorporate multiple droplet pairs rather than extended droplet networks. 


\subsubsection{Useful manipulations of droplets}

Before discussing the functional properties of droplet pairs, and subsequently networks, it is worth recalling several useful manipulations that can be performed with them. DIBs can be dissociated (e.g. by pulling). The droplets can be reassembled or used to form new pairs ${ }^{3,24}$. DIBs can be perfused, from one side of the bilayer at least ${ }^{44,45,50}$. A donor droplet can be fused with one of the droplets in a pair to introduce a new reagent or to manipulate a reagent's concentration ${ }^{44,48,49}$. Proteins, including membrane proteins, can be produced by IVTT (cell-free synthesis) within droplets ${ }^{16,24,72,74}$. The bilayer area can be increased or decreased, e.g. by pushing or pulling on an electrode ${ }^{21,31,32}$. Finally, droplet-based devices can be robust; recently, a DIB-based sensor was used at the top of Mount Fuji ${ }^{2}$.

\subsubsection{Sensing and screening}

\subsubsection{Mechanosensors}

Sarles, Leo and their associates have addressed mechanosensation with droplet technology. The vibration of an artificial "hair" in one droplet of a pair, caused by air flow, produced current noise arising from modulation of the DIB area and hence its capacitance ${ }^{35,93}$ (Figure 19A). The vibration and hence the electrical signal can be transmitted along a droplet chain ${ }^{94}$. The activation of the mechanosensitive channel protein MscL has also been investigated in a set-up in which a DIB is subjected to cyclic compression and decompression with a piezoelectric driver ${ }^{95,96}$. Gating occurred at maximum compression, and was ascribed to elevated bilayer tension. However, an understanding of the gating forces in an oscillating system in which relaxation effects must be accommodated remains incomplete ${ }^{95,97}$. Nonetheless, the use of DIBs offers a potentially powerful approach for understanding how bilayer mechanics control the gating of mechanosensitive channels. Notably, membrane bending (another potential driving force for gating) can be produced by using two droplets of different diameter, and hence maintaining a pressure differential as predicted by the Young-Laplace equation ${ }^{97}$. A chemically-activatable mutant of MscL has also been shown to open and mediate the transport of a fluorophore across DIBs ${ }^{98}$.

\subsubsection{Translocation assays}

The Holden group has examined protein translocation across a DHB. If the translocated protein is a channel blocker, such as the lethal factor of anthrax toxin, it can be detected in a second DIB assay ${ }^{26}$ (Figure 19B). Alternatively, a translocated enzyme for which a highly sensitive assay is available, such as horseradish peroxidase (HRP), can be detected in the acceptor droplet $^{43,99}$. Recently, the bacterial lactose transporter LacY has been assayed in a DIB by using a fluorescent substrate analog ${ }^{72}$. It would be advantageous if a more general method of detecting translocated materials, such as mass spectrometry, could be exploited. 


\subsubsection{Chips for screening membrane proteins}

It is vital to develop techniques to screen the biological properties of membrane proteins, because of their importance in functions including signal transduction in the nervous system ${ }^{100}$, muscle contraction ${ }^{101}$, the transport of nutrients and ions ${ }^{102}$, T-cell activation ${ }^{103}$ and insulin release ${ }^{104}$. Current biochemical screening platforms incorporate robotic liquid-handling systems to screen the activities of thousands of chemical compounds with in vitro assays on protein targets such as enzymes ${ }^{105}$. They usually rely on microplate readers, which require $1 \mu \mathrm{L}$ to $1 \mathrm{~mL}$ of sample. Additionally, microarrays provide information on protein abundance, post-translational modifications, splicing events, polymorphisms, cellular and subcellular localization, and the interactions between proteins ${ }^{106}$. However, these approaches cannot record the activities of membrane proteins in lipid bilayer environments ${ }^{107}$.

Patch-clamping allows the study of membrane proteins in native membranes, however, this technique is technically challenging ${ }^{107}$. Although patch-clamping is generally performed at low-throughput, there has been progress towards high-throughput measurements ${ }^{108,}$ 109. Alternatively, various types of lipid membranes have been developed to study reconstituted membrane proteins in vitro, including black lipid membranes (BLM), supported lipid bilayer membranes (s-LBM), air-stable lipid bilayer membranes (as-LBM), hybrid lipid bilayer membranes (h-LBM) and polymer-cushioned lipid bilayer membranes $(\mathrm{pc}-\mathrm{LBM})^{110}$. However, little work has gone into developing functional screening platforms using these systems. Notably, microelectrode cavity arrays $^{111}$ have been developed into the 16-channel Orbit device (Nanion Technologies). Several droplet-based chips or devices for in vitro screening of membrane proteins have been devised and are described in this section. These work either on a sequential or a parallel basis. However, it should be said that parallel lipid-bilayer-based devices have to this point either not been fully implemented or have severe restrictions.

\subsection{Sequential screening}

Sequential processes are based on the ability to break and reform DIBs ${ }^{3,24}$. In a proof of principle, three separate droplets containing buffer or one of two different pore blockers were attached to a common electrode ${ }^{3}$. A mobile droplet containing $\alpha \mathrm{HL}$, on a separate electrode, was used to form a DIB with each droplet sequentially. Upon contact, several copies of the $\alpha \mathrm{HL}$ pore inserted into the bilayer and were detected as stepwise increases in the ionic current under an applied potential. The binding of the blockers was detected as superimposed transient current blockades. Importantly, there was no carry over of a blocker when a new DIB was formed.

The previous approach ${ }^{3}$ was expanded by making a chip with an array of droplets (Figure 20A). The chip containing 16 droplets, each loaded with a different channel blocker, was scanned manually with a mobile droplet carrying the $\mathrm{Kcv} \mathrm{K}^{+}$channel making one bilayer at a time ${ }^{23}$. The $\mathrm{K}^{+}$channel was made by IVTT and was not purified before forming the mobile droplet ${ }^{23}$. 
Instead of manual DIB formation, a microfluidic device was used to trap one droplet on an electrode and repeatedly exchange a second droplet impaling it on a second electrode ${ }^{42}$ (Figure 20B). The system was used to measure the affinity of the $\alpha \mathrm{HL}$ pore for the blocker $\gamma$-cyclodextrin. An automated gravitydriven device was devised for the formation of DIBs, which might be useful for identical repeated measurements on a channel or pore ${ }^{112}$. Droplet exchange has also been automated in a mechanical "split and contact" device, which allows the repeated formation of bilayers by using a rotating electrodecontaining unit ${ }^{75}$. Controlled DIB formation and dissociation has also been demonstrated by using dielectrophoresis on planar microelectrodes ${ }^{25}$. Simultaneous controlled formation and dissociation of multiple DIBs has been performed with a mechanically operated chamber ${ }^{113}$, but as yet the device has not been used to perform measurements of function.

An individual DIB can also be employed continuously when solution exchange is enabled. For example, the affinity of the TRPM8 "cold" channel for an activator and a blocker was determined by flowing test solutions across one face of a DIB reinforced by an aperture in a Delrin partition ${ }^{46}$. In this format, a single droplet containing TRPM8 in an upper oil phase forms a bilayer with a lower bulk aqueous solution, which can be exchanged. The speed of the solution exchange could be increased by using a hydrogel-stabilized droplet, which was dipped before use into liposomes into which TRPM8 had been incorporated $^{53}$. The use of microfluidics has also proved useful for solution exchange in DIBs. Two droplets, placed in two adjoining wells, formed a bilayer through an aperture, which restricted the bilayer size. One of the two wells incorporated a microfluidic inlet and outlet, which allowed solution exchange within seconds ${ }^{50}$.

A droplet-fusion system has been devised to introduce new material into one droplet of a droplet pair ${ }^{48}$ (Figure 20C), which has allowed the same Wza pore to be subjected to a sequence of conditions. A DIB was formed across an aperture ( $200 \mu \mathrm{m}$ in diameter) in a Teflon film, which restricted the size of the bilayer and provided stability. Electrical recordings of a pore in the DIB were then made with electrodes inserted into each droplet. Additional reagents were then added to one of the droplets forming the DIB from a third droplet suspended on a silver wire. Forcing the third droplet and the wire into the recipient droplet caused fusion and the mixing of contents. The DIB was not disrupted, allowing electrical recording to be continued.

\subsection{Parallel recording}

Several chips have been devised with the goal of performing automated parallel electrical measurements. However, for the most part, while these devices are compatible with high throughput assays, such assays have not been executed because a multichannel amplifier was not available or because the loading of compartments was too awkward or impractical. For example, thousands of individual DIBs were made by robotic pipeting of $1 \mu \mathrm{L}$ droplets onto horizontal oil-buffer interfaces. However, only one current recording was made at a time ${ }^{114}$. The same authors also formed DIBs by bringing a droplet to an interface on a pin tool or by partial expulsion from a micropipet ${ }^{115}$. Again, 
parallel recordings were not performed. A fully functional 16-channel DIB system has been devised in which robotic pipetting is used to control all of the components in each of the recordings ${ }^{47}$. The robot made the DIBs, reconstituted ion channels in an oriented fashion (by vesicle fusion) and added drug molecules. A plastic partition was used to reduce the contact area (and hence the noise) and to stabilize each droplet pair.

Given the difficulty of separately electrically addressing hundreds of wells, the optical recording of channel or pore activity is an inviting prospect, where it can be applied. For example, $\mathrm{Ca}^{2+}$ diffusion through ensembles of $\alpha \mathrm{HL}$ pores was measured in a 15-DHB format by using the dye fluo-8 in the recipient droplet $^{116}$ (Figure 21A). Tonooka and colleagues carried out 36 similar measurements in parallel after depositing a large lipid-bounded aqueous droplet on top of an array of smaller lipid-bounded aqueous droplets ${ }^{117}$ (Figure 21B). Similar approaches have been used with alternative bilayer arrays ${ }^{118}$ and implemented at the single pore level ${ }^{119} \cdot \mathrm{Ca}^{2+}$ flux through hundreds of individual $\alpha \mathrm{HL}$ pores under an applied potential can also be monitored in the DHB format ${ }^{120}$ (Figure 21C) (see Section 3.1.4). The dividing line between conventional bilayers and DIBs is not sharp, and bilayers can be made in which almost all the oil is removed by absorption ${ }^{6}$, dissolution ${ }^{121}$ or physical separation $^{122,123}$, and these processes can also yield bilayer arrays ${ }^{121-123}$.

\subsection{The ideal screening device}

The ideal screening device would incorporate thousands of separately electrically addressed wells, each of which would contain a different channel or pore in the bilayer or a different analyte, or concentration of analyte, in the aqueous phase. Preferably, activity would be recorded at the single-molecule level. Commercial chips $(13 \times 5.5 \mathrm{~mm})$ with hundreds of active wells are used by Oxford Nanopore (www.nanoporetech.com) for single-molecule DNA sequencing ${ }^{124}$. Each well contains a separate electrically-addressed aqueous volume. A triblock copolymer (rather than lipid) is applied across the surface of the chip in an oil phase, which is then largely replaced by an upper aqueous phase to form numerous compartments from which electrical signals can be recorded from individual pores incorporated at each interface. In the simplest manifestation of the chip, identical pores are present in each well and the same DNA sample is applied to each one in the upper aqueous phase. Potentially, in a similar chip, different aqueous droplets might be placed in each well to allow the simultaneous examination of hundreds of different analytes (or say a library of ion channels), thereby permitting screening at moderate throughput in small volumes under multiple conditions.

\subsubsection{Chemical reactions in compartments}

There has been considerable interest in generating microreactors for chemistry and biochemistry from aqueous droplet networks. These might allow a reaction to take place in one compartment before the product moves to the next for a second reaction step. This process could be incorporated into a sensing device for small molecules with, for example, a fluorescence output. Transport between droplets could occur by diffusion through the DIB or by 
translocation through protein pores. A droplet system might facilitate step-wise microscale syntheses of complex molecules that require several different reaction environments. Diffusion in a two-droplet system will probably not be limiting; a small molecule diffuses $250 \mu \mathrm{m}$ in $\sim 15 \mathrm{~s}$. Nonetheless, transport from one droplet to another might be promoted by using a stimulus, such as voltage.

\subsubsection{Conventional chemistry}

Microfluidics approaches were used by Bai and colleagues to obtain surfactant-stabilized $A B$ droplet pairs with different reagents in each droplet ${ }^{59}$. The authors observed fluorescein or $\mathrm{H}_{2} \mathrm{O}_{2}$ diffusion across the interface. In the case of $\mathrm{H}_{2} \mathrm{O}_{2}$, the recipient droplet contained Amplex Red ( $\mathrm{N}$-acetyl-3,7dihydroxyphenoxazine) and HRP, which catalysed the formation of the fluorescent product resorufin. Carreras and colleagues demonstrated a similar process $^{85}$ (Figure 22A). Elani and colleagues investigated enzyme-catalyzed reactions in two-droplet multisomes. In one droplet, glucose was converted to D-gluconolactone and hydrogen peroxide, catalyzed by glucose oxidase. As observed in the earlier work, the peroxide diffused into the second droplet and oxidized Amplex Red ${ }^{125}$. This work has been extended to a 3-droplet system (see Section 3.4.2). A simple reaction in which ethanolamine diffused through a DIB to react with a fluorogenic reagent was demonstrated in two-droplet multisomes generated in a microfluidics system ${ }^{63}$.

\subsubsection{Oscillating reactions and chemical waves}

The Belousov-Zhabotinsky (BZ) oscillating reaction has been demonstrated in single droplets and $\mathrm{DIBs}^{84,126,127}$. In the $\mathrm{BZ}$ reaction, the ratios of oxidation states of reaction intermediates oscillate. The $B Z$ reaction is ideal for droplet studies, because it can be monitored optically ${ }^{127}$ (Figure 22B). When the BZ reaction occurs in individual droplets they undergo shape changes (e.g. elongation and contraction along an oil-filled trench) ${ }^{84}$. The oscillating shape change has been suggested to arise from changes in the surface tension of the droplet as the concentrations of components rise and fall. In BZ-containing droplet pairs, the oscillating chemical reaction moves through the DIB without causing rupture ${ }^{126,127}$ in a manner that depends on the relative volumes of the droplets $^{127}$. The propagation of the $B Z$ reaction across a lipid bilayer must occur because the excitatory and inhibitory components of the reaction mixture traverse the bilayer, although details of this process are not clear ${ }^{126}$. The BZ reaction can also produce self-propulsion in asymmetric droplet pairs $^{126}$, a phenomenon worthy of further exploration. The BZ reaction has also been demonstrated in more elaborate droplet networks, which are described later (see Section 3.2.4).

\subsubsection{DHB technology}

In droplet hydrogel bilayers (DHB, Figure 1C, D), a lipid-coated droplet in oil contacts a lipid monolayer on a flat hydrogel surface. DHBs offer the ability to simultaneously record electrical current and fluorescence (by total internal reflection $)^{11,37,38}$. By using a $\mathrm{Ca}^{2+}$-sensitive fluorophore, ionic currents can be 
detected from numerous pores simultaneously ${ }^{37}$, which may allow highly parallel recording for stochastic sensing and nanopore DNA sequencing ${ }^{120}$. Arrays of hydrogel pillars allow the robotic loading of different samples onto a single "chip"120 (Figure 21C). However, in the present format, the limited field of view has allowed only 4 pillars ( 200 pores) to be visualized at once. A K ${ }^{+}$sensitive fluorophore has also been employed ${ }^{128}$. The DHB approach has been used to track the diffusion of lipids and proteins in bilayers ${ }^{129}$, to study the electroporation of bilayers ${ }^{128,130}$, to determine bilayer specific capacitance and manipulate bilayer area ${ }^{32,131}$, to investigate pore assembly ${ }^{70,132}$, count the subunits in pores by photobleaching ${ }^{133}$, as well as to examine a wide variety of channels and pores ${ }^{69,134}$. Recently, the lipid composition of preformed DHBs has been altered by titrating lipids into the oil phase ${ }^{135}$. To achieve rapid equilibration (minutes), the DHB was heated to above the phase transition temperature of all the lipid components (in this work $46^{\circ} \mathrm{C}$ ). DHBs can also be imaged by interferometric detection of differential light scattering, which is a versatile label-free technique with high time resolution that has, for examples, been used to track lipid nanodomains ${ }^{136}$. Clearly, variations of the DHB approach will continue to make important contributions to membrane biophysics.

\subsection{Functional properties of 2D droplet networks}

\subsubsection{Electronic devices from 2D droplet networks}

Bioelectronic materials have been constructed that interact with living systems, including a biomimetic neuron ${ }^{137}$ and an artificial retina ${ }^{138}$. Most of these systems have included rigid semiconductor components ${ }^{139}$. By contrast, natural biological materials are generally soft and flexible, so it is important to mirror these properties in bioelectronic devices in order to better match the mechanical properties of tissues ${ }^{140}$. In this way, inflammation and scarring, which would limit the functionality and life expectancy of a device, are lessened $^{141}$. The employment of functional networks of communicating $\mathrm{pL}$ to $\mathrm{nL}$ aqueous compartments may pave the way towards soft biological electronics that can be interfaced with natural systems.

Soon after the introduction of DIBs, steps were taken to demonstrate that droplet networks can behave as electrical circuits ${ }^{31}$. An electrical modelling approach was used to describe the current flow through simple droplet networks where the lipid bilayers served as capacitors and membrane pores (such as $\alpha \mathrm{HL}$ ) incorporated into the bilayers acted as resistors. Of course, in the case of droplet networks, current is carried by ions rather than by electrons. Even in three-droplet systems, unusual pore blocking events were observed that are not seen in individual DIBs. These events were readily rationalized on the basis of current simulations, and it was argued that the approach might be extended to far more complex droplet networks.

Following this initial study, the applications of a mutant $\alpha \mathrm{HL}$ pore that acts as a "diode" were explored ${ }^{142}$. The $\alpha H L$ mutant (7R- $\left.\alpha \mathrm{HL}\right)$ contains forty-nine positively charged side chains projecting into the lumen of the transmembrane $\beta$ barrel, which causes it to open and conduct current only at positive applied 
potentials. Electrical devices were made with the 7R pore by incorporating it into DIBs in droplet networks in a vectorial fashion, which was achieved firstly because the $\alpha \mathrm{HL}$ heptamer inserts into bilayers exclusively stem first, and secondly because the majority of insertion occurs soon after a DIB is formed, allowing fresh vectorial insertion when a new droplet is introduced into a network. With this approach a 3-droplet current limiter, a 3-droplet half-wave rectifier and a 4-droplet full-wave rectifier were prepared (Figure 23A). The development of droplet diodes, the basic unit of microprocessors, suggests that the capacity to compute can be built into droplet networks. While such devices are unlikely to compete with existing computers, the ability to make simple internal calculations in droplet networks is an intriguing possibility.

\subsubsection{Power generation in 2D droplet networks}

Three- and six-droplet biobatteries have previously been constructed, based on a concentration cell ${ }^{3}$. These networks function by incorporating an engineered anion-selective $\mathrm{\alpha HL}$ pore into a DIB separating two droplets containing different concentrations of $\mathrm{NaCl}$ (Figure 23B). Current was generated across the droplet biobatteries in the absence of an externally applied potential, because $\mathrm{Cl}^{-}$diffused through the pore, while $\mathrm{Na}^{+} \operatorname{did}_{\text {not }}{ }^{3}$. Because the battery relied on a salt concentration gradient, it ran for only less than an hour, before ion and water transport across the bilayer, the latter by osmosis, balanced the salt concentrations. Lavan and colleagues have analyzed the biobattery system to take into account chemistry at the $\mathrm{Ag} / \mathrm{AgCl}$ electrodes $^{143}$. To make use of an external energy source, the light-driven proton pump bacteriorhodopsin (bR), from Halobacterium salinarum, was used to generate current in a droplet system ${ }^{3}$ (Figure 23C). bR is a membrane protein, which actively transports one proton across a bilayer per photon of light absorbed, thereby generating an ionic current provided the protein is vectorially inserted.

The means have not yet been devised to couple power generation to functional properties of printed droplet networks, such as a reversible shape change. In this regard, the use of light is especially interesting, because it might act both as an external signal and a power source. Structured illumination might be used to switch power on-and-off in selected regions of a network (see Section 3.3.2.3). Patterned current generation has been established within a $4 \times 4$ array of bR-containing droplets, each interfaced with a common hydrogel structure through a lipid bilayer ${ }^{144}$ (Figure 24). Electrodes inserted into the droplets measured the light-driven proton-pumping activity across the sixteen interface bilayers simultaneously. By measuring the electrical current generated in each 'pixel', grey-scale images and patterns of light moving across the device were detected. While the device can be seen as a simple soft retina, such a structure might also be embedded within printed droplet networks to functionalize them.

\subsubsection{Logic operations in 2D droplet networks}

A logic gate is a practical implementation of a Boolean decision-making device. Kawano, Takeuchi and colleagues have developed logic gates from 
droplet networks, a form of biological computing, as described in several conference abstracts. For example, Yasuga and colleagues carried out AND, OR and NOT operations by using various combinations of "droplet-boxes" ${ }^{145}$. A single droplet was stabilized in each box, which had two windows with apertures for DIB formation. The boxes were either "on" (contained $\alpha H L$ ) or "off" (no $\alpha \mathrm{HL}$ ). Inoue and colleagues used a droplet split-and-contact device ${ }^{75}$ to carry out OR and NOT functions that depended on the contents of input droplets that were fused on demand to a central droplet by using a voltage pulse $^{146}$. The outputs depended on whether the various droplets contained the pore-forming protein $\alpha \mathrm{HL}$ with various single-stranded DNAs (ssDNAs). Current through the $\alpha H L$ pores could be temporarily blocked when ssDNA was present or permanently blocked by double-stranded DNA (dsDNA), formed when two complementary ssDNAs from two droplets hybridized. In published work, a NAND (negative-AND) gate was developed with a four-droplet network, where three droplets, two input and one output, were each connected to a common central droplet ${ }^{147}$ (Figure 23D). A long ssDNA was present in the central droplet and two short ssDNAs, complementary to each end of the long strand, were each placed in an input droplet. The long ssDNA could translocate to the output droplet when either both short sSDNA were not present or when only one was present in the input droplets. However, when both ssDNAs were present a complete dsDNA formed, which stopped the long ssDNA from translocating, constituting a NAND operation. An interesting aspect of this work was the use of the large streptolysin O pore to speed up the translocation of ssDNA from the input droplets. The $\alpha \mathrm{HL}$ pore, which cannot transport dsDNA, was used at the output interface.

These droplet network logic gates function slowly (e.g. in minutes), because they are based on several time-consuming events, including the translocation of DNA through pores. Therefore, it is doubtful whether droplet-based devices can provide more powerful computing than electronics, or eventually quantum computing or DNA computing. However, droplet networks possess certain advantages. For example, they are soft structures made from biocompatible materials, which might allow them to interact with living cells to sense metabolites or deliver drugs when certain conditions requiring simple internal computation are met.

\subsubsection{Chemical reactions in 2D droplet networks}

The chemistry performed in two-droplet systems (see Section 3.1.3) might be extended to droplet chains and networks to allow more complex multistep chemical reactions to occur in a sequence of droplets. Surprisingly little work has been performed along these lines. Diffusion of $\mathrm{H}_{2} \mathrm{O}_{2}$ from a single droplet at the end of a linear chain of four aqueous droplets ( 100 $\mu \mathrm{m}$ diameter) containing HRP and Amplex Red has been demonstrated ${ }^{62}$ (Figure 24A). Fluorescence from the HRP-product resorufin was generated along the entire chain. Consecutive enzymatic conversions have been executed in 3-droplet multisomes $^{125}$ (see Section 3.4.2).

More sophisticated chemistry in chains and networks will require manipulations such as the entrapment in droplets of reagents and catalysts 
(e.g. on beads) and enzymes, the introduction of reagents by droplet fusion, the active movement of reaction products (e.g. in an electric field), the execution of combinatorial chemistry by the mixing and matching of droplets and the trapping of final products in terminal droplets. The terminal droplets might be removed by DIB dissociation and the contents used, e.g. in pharmacological assays. Diffusion rates along droplet chains are an important issue. The mean time taken for a small molecule to traverse a chain of ten 200 $\mathrm{nL}$ droplet is several hours, which is reduced to a few minutes with $50 \mathrm{pL}$ droplets, assuming rapid passage through pores in the DIBs.

The $B Z$ reaction has also been observed in droplet chains. BZ waves can migrate not only across a single bilayer, but also from one end of a linear chain of droplets to the other, crossing multiple DIBs in the process ${ }^{84,148}$ (Figure 25B). The chemical waves travel faster than molecular diffusion over long distances. More complicated 2D BZ patterns have been shown to form branched junctions and travel as waves and spirals through sheets of hundreds of DIB-connected droplets ${ }^{126}$ (Figure 25C). Components of the reaction must pass through $\mathrm{DIBs}^{126}$, but the identity and rate of diffusion of these "transmitters" is unknown.

\subsection{Functional properties of 3D droplet networks}

\subsubsection{Functional properties of small 3D droplet networks}

The functional properties of small 3D droplet networks have been hardly examined, and deserve further exploration. Conductive pathways within droplet pyramids were created by using the $\alpha \mathrm{HL}$ pore, and the directional transmission of ionic currents and the diffusion of fluorescent dyes were examined $^{68}$. 3D structures provide more compact pathways than 2D structures with respect to the execution of any particular electronic function or logic operation. They also offer the potential for more complex circuitry. Further, 3D structures are more robust than linear arrays or sheets of droplets. They might also be used as building blocks for larger networks ${ }^{68}$.

\subsubsection{Functional properties of large 3D droplet networks}

The ability to 3D print large patterned structures consisting of droplets with different contents has opened up the prospect of producing novel soft materials, which might be used, for example, as synthetic tissues. We outline progress on the attainment of emergent properties from large droplet networks and the ability to control networks with external stimuli.

\subsubsection{Electrical signaling in 3D droplet networks}

It had been shown that 2D networks of tens of aqueous droplets conduct an electrical signal if $\alpha \mathrm{HL}$ pores are included in every droplet in a pathway ${ }^{3,31}$. By using the 3D printer, electrical communication through an order of magnitude more DIBs has been achieved, by incorporating $\mathrm{aHL}$ into the droplets of a cuboid network ${ }^{15}$ (Figure 26A-D). To characterize these networks electrically, electrodes were inserted into two large manually-pipetted droplets, which were 
then brought into contact with two opposite sides of the cuboid. The electrode droplets formed $\alpha \mathrm{HL}$-containing bilayers at the interfaces with the network and, when a potential was applied, an electrical current was observed, demonstrating that $\mathrm{aHL}$ pores had inserted into DIBs throughout the entire 3D droplet network. Electrical communication was also demonstrated through defined pathways in 3D droplet networks, taking advantage of the patterning capabilities of the 3D printer (Figure 26E-H). Two types of droplets, either containing or not-containing $\mathrm{\alpha HL}$, were incorporated into a network. Hundreds of $\mathrm{aHL}$-containing droplets were patterned in a directional pathway from one side of the network to an adjacent side, with the other droplets containing no protein. When electrode droplets were applied to form bilayers at both ends of the pathway, electrical current signals were observed upon the application of a potential. This demonstrated that, by using the 3D printer, directional electrical communication through droplet networks can be established. Transmission of the signal was faster than the acquisition rate of the amplifier used (100 $\mu \mathrm{s})$ and therefore at $>10 \mathrm{~m} \mathrm{~s}^{-1}$ at least as fast as neuronal signaling in mammals $^{149}$.

\subsubsection{Folding 3D droplet networks}

As well as directional communication through networks, 3D printing has allowed the creation of droplet networks that can undergo shape changes after printing ${ }^{15}$. This "4D-printing", an emerging field in its own right, allows the generation of geometries that cannot be printed directly ${ }^{150}$. Macroscopic movement was driven by osmosis. When droplets in a pair contain different salt concentrations, water diffuses across the DIB to equilibrate the salt concentrations, which causes one droplet to shrink and the other to swe $\|^{20,151}$ (Figure 27A). For example, rectangular networks were printed that contained two vertical strips, one with droplets containing a high salt concentration and the other with droplets containing a low salt concentration ${ }^{15}$ (Figure 27B). Following printing, the low salt droplets shrank and the high salt droplets swelled. This caused the rectangular network to fold into a circle, in which the two ends of the structure touched and formed bilayers.

Through careful design, it was also demonstrated that networks can fold out of the horizontal plane against gravity ${ }^{15}$ (Figure 27C). A shape composed of four petals was printed onto PMMA; the top layers contained low-salt droplets (8 $\mathrm{mM} \mathrm{KCl})$ and the bottom layers contained higher-salt droplets $(80 \mathrm{mM} \mathrm{KCl})$. Following printing, the movement of water caused the top layers to shrink and the bottom layers to swell, which forced the petals to fold upwards and the edges of each petal to touch, forming a hollow sphere. The outside of the sphere contained what were originally the high-salt droplets, and the inside of the shell the shrunken low-salt droplets. Hollow geometries, like these, would have been impossible to construct with the printer alone. Calculations demonstrated that the force generated from osmosis was orders of magnitude greater than that required to lift droplets against gravity. The 3D movement devised for aqueous droplet networks mirrors the non-muscle hydraulicallyactuated movement seen in plants and fungi ${ }^{152,153}$. However, at the present stage of development, it is slower. For example, formation of the sphere required 8 hours. 
Important changes were made to the printing process to allow networks to fold $^{15}$. Previous networks had been printed on a glass surface, which is adhesive towards the droplets. For folding, printing was performed on PMMA, which is not adhesive. Because the osmotic water flow began as soon as DIBs formed in the network, the speed of printing was increased, relative to the folding time, so that the extent of folding was minimized during printing. Minimizing the difference in salt concentration between the two types of droplet reduced the rate of osmosis. If osmosis were too fast, then significant folding would occur before printing was finished. For instance, water transport was complete within 1 hour with two $200 \mathrm{~nL}$ droplets containing $1 \mathrm{M}$ and 250 $\mathrm{mM} \mathrm{KCl}$. In addition, the printing time was reduced by using shorter delays between the ejection of each droplet, although this slightly reduced the patterning fidelity of the printer. Furthermore, larger droplets were used, as they showed slower relative volume changes, because the rate of water transport is proportional to the DIB area. The printing of additional layers of droplets reduced the buckling and fracturing encountered as the networks deformed, most probably because the migration of droplets filled incipient gaps in the structures (Figure 27C-E). The osmotic folding of droplet networks was modeled by a simulation in which adjacent droplets were coupled by Hookean springs and the osmotic flow of water, which altered the center-tocenter separation of the droplets, was based on experimentally determined values.

These 3D printed droplet networks can only change shape once; movement ceases when osmotic balance is achieved. Reversible movement would be of considerable interest. By using molecular dynamic simulations, a theoretical study has demonstrated that, by adding an osmotic interaction with the surrounding environment, dynamic osmotic networks might be created ${ }^{154}$. The osmolyte concentration inside and outside the network and the number of droplets comprising the network were analyzed, and particular combinations of these produced theoretically reversible folding. Therefore, it might be possible to use solutions (e.g. of temperature-sensitive polymers ${ }^{155}$ ) that are expected to be capable of changing osmolarity to generate reversible shape changes. Networks that fold reversibly would be a first step towards soft robots that can hold and release objects, or swim or walk ${ }^{156,157 .}$

\subsubsection{Controlling 3D droplet networks}

The control of droplet networks post-printing is an important step in the development of the technology, particularly if control can be implemented with a patterned stimulus. The previously described examples of long-range electrical signaling (see Section 3.3.2.1) and shape change (see Section 3.3.2.2) in large droplet networks cannot be controlled post-printing. The properties were defined before printing; once a network was printed, there was no way to alter its functional properties. Additionally, these examples only utilized aqueous droplets containing simple salt solutions or a single purified protein. It is also of interest to introduce more complex controllable reactions into extended droplet networks. 
The first example of post-printing control of 3D-printed aqueous droplet networks incorporated a light-activatable IVTT protein expression system ${ }^{16}$. Aqueous droplets containing the expression system made protein encoded by a DNA template, which was also incorporated into the droplets. The expression system contained the minimum number of purified components required for protein synthesis ${ }^{158}$. The aqueous droplets were termed synthetic cells, as they were able to perform a significant biological function within individual compartments (Figure 28). The synthetic cells were externally controlled by UV light by means of light-activated DNA (LA-DNA) ${ }^{16}$, made by covalently linking a DNA promoter to seven biotins, each containing a photocleavable linker ${ }^{159}$. Monovalent streptavidin ${ }^{160}$ was subsequently attached to each biotin, which sterically blocked RNA polymerase from binding to the LA-DNA. Upon irradiation, the linkers were cleaved, enabling RNA polymerase to access the DNA and transcribe it into RNA, which was translated into protein. An important advantage of this LA-DNA was its tightly regulated off-state, from which negligible protein was expressed before UV activation. Such tight control is not usually found in light-activated systems ${ }^{161}$, and it prevented protein expression during the slow printing process.

To 3D-print aqueous droplet networks composed of these light-activatable synthetic cells, it was necessary to improve the stability of the networks. With a high concentration of protein inside the droplets (from the IVTT system), DIB formation was slow and once formed the droplets tended to fuse ${ }^{24}$. In a 3Dprinted network of hundreds of droplets, this would result in imperfect packing. Several lipid mixtures and oil compositions were tested in attempts to produce well-defined, stable droplet networks. Lipids with PEGylated headgroups have been used extensively to protect bilayers from biological components ${ }^{162}$. A mixture of $10-15 \%$ 1,2-dipalmitoyl-sn-glycero-3-phosphoethanolamine-N[methoxy(polyethylene glycol)-2000] (DPPE-mPEG2000) with DPhPC, in a 1:1 mixture of hexadecane and silicone oil, resulted in long-term stability of DIBs in droplet networks containing the IVTT system ${ }^{16}$. Reduction of the 1:1 ratio, by increasing the silicone oil concentration, increased the contact angle of the DIBs. At the intensity of UV light used to activate the LA-DNA, there was no appreciable loss of protein synthesis activity.

Cuboid networks were printed that were composed of hundreds of synthetic cells, each containing LA-DNA encoding a fluorescent protein ${ }^{16}$ (Figure 28AC). When the printed cuboids were illuminated with UV light following printing, protein expression was observed throughout the network. This demonstrated post-printing control of a biological process inside droplet networks. Cuboid networks were also printed with a 3D pathway that comprised synthetic cells containing $\alpha \mathrm{HL}$ LA-DNA, while the remaining compartments contained no DNA (Figure 28D-F). Due to the stability afforded by the PEG lipids, electrodes could be interfaced with the sides and top of the network. Following printing and illumination, electrical signals were detected only when the electrodes were placed at either end of the pathway. These 3D-printed networks of synthetic cells were termed synthetic tissues, as they demonstrated an emergent function beyond that achievable with a collection of noncommunicating synthetic cells. 
By combining an IVTT system with LA-DNA, controllable biological function was included within aqueous droplet networks, which was activatable in a patterned manner either by printing the desired pattern or by using patterned irradiation ${ }^{16}$. Light is an ideal means to control synthetic tissues, because it can penetrate through an entire network. Indeed, synthetic tissues absorb and scatter less light than natural tissues. Furthermore, light is an external stimulus that does not require the material to be permeable, e.g. to small molecule activators for use with designer receptors (the DREADD approach) ${ }^{163}$. Irradiation may be a useful means of patterning when methods of network generation are used, for instance microfluidic processes, with which it is difficult to arrange different classes of droplets. Microfluidically generated droplets, stabilized by the surfactant Span 80 and containing a photo-caged fluorescein, have been patterned by using 2D illumination ${ }^{164}$. By using a lipid, instead of a surfactant, microfluidically generated DIB networks might be patterned in a similar manner.

\subsection{Functional properties of droplet networks in an aqueous environment}

A long-term goal is to apply droplet networks in biology and medicine, which requires that they be transferred to an aqueous medium (see Section 2.5) so that they might be integrated with natural tissues or secrete and receive effector molecules.

\subsubsection{Interactions of multisomes with the surrounding aqueous environment}

Multisomes have been prepared with functionalized bilayers containing $\mathrm{pH}-$ or temperature-sensitive lipids, by using a manual procedure (see Section 2.5). When triggered, the contents of the internal aqueous compartments were expelled into the aqueous environment ${ }^{89}$ (Figure 29). Such constructs might be developed as sophisticated drug delivery systems (see Section 4). $\mathrm{pH}$ sensitive multisomes incorporated the lipid 1,2-dioleoyl-sn-glycero-3phosphoethanolamine (DOPE), which does not favor bilayer formation. The structures were stabilized with oleic acid. Protonation of the oleic acid occurred when the $\mathrm{pH}$ was decreased, which triggered the rupture of the bilayers between the internal droplets and at the interface with the aqueous environment. The temperature-sensitive bilayers contained 1,2-distearoyl-snglycero-3-phosphocholine (DSPC) and DPhPC. Multisomes prepared with this mixture ruptured at $\sim 44^{\circ} \mathrm{C}$, due to a phase separation of the lipids ${ }^{89}$. The lifetimes of these prototype triggerable multisomes before activation ranged from hours to about a day, which is sufficient for drug delivery applications, but requires improvement for longer term implants (see Section 4). DIBs containing diyne lipids become porous to small molecules upon UV irradiation, and might also be used for triggered release ${ }^{165}$.

Manually prepared multisomes (see Section 2.5) are also able to communicate electrically with their environments through $\alpha \mathrm{HL}$ pores incorporated into the encapsulated droplets ${ }^{89}$. The insertion of $\alpha \mathrm{HL}$ into the external bilayer allowed the measurement of an ionic current travelling from the interior of a multisome to the external solution. Alginate-encapsulated droplet networks (Figure 
$17 \mathrm{E}, \mathrm{F})$ were similarly able to communicate with the external environment ${ }^{56}$. In this case, $\alpha \mathrm{HL}$ became incorporated into the external bilayers of multisomes after diffusion from the external solution through the protective alginate layer. An ion flux was measured between an electrode inserted into one of the internal droplets and an electrode in the hydrogel shell. Electrical communication has also been demonstrated in assemblies of aqueous droplets contained within agarose-encased oil drops ${ }^{57}$. Capacitance measurements confirmed the integrity of the bilayers, and the transport of ions and dye molecules occurred through bilayers into which the ClyA pore had been incorporated. Electrical communication between droplets in separate oil drops, 100 to $200 \mu \mathrm{m}$ apart within the same agarose block, was also demonstrated.

\subsubsection{Chemistry and biochemistry in multisomes}

Chemistry in droplet pairs (see Section 3.1.3) and droplet networks (see Section 3.2.4) in oil have been discussed. Reaction sequences have also been performed in multisomes in an aqueous environment. Protein pores were incorporated into the DIBs in multisomes generated with the phase transfer method $^{90}$, allowing a sequence of three enzymatic reactions to take place as small molecules diffused through bilayers and pores in a network ${ }^{125}$ (Figure 30) (see Section 3.2.4). Lactose was converted to glucose by lactase, and glucose to D-gluconolactone and hydrogen peroxide by glucose oxidase. Finally, the peroxide oxidized Amplex Red to the fluorescent resorufin catalyzed by HRP. The compartmentalization of these reactions was demonstrated by encapsulating each of the three enzymes in 2- and 3-droplet multisomes $^{125}$. Glucose could be transferred between compartments through $\alpha H L$ pores, while hydrogen peroxide was able to diffuse rapidly through DIBs. Reagents could also be introduced through pores from the external medium. A flaw of the system was the ability of the reactants to move into inappropriate compartments or into the surrounding buffer. More recently, in a multisome droplet pair assembled through microfluidics, the membrane-permeant primary amine, ethanolamine, passed through the DIB into the second droplet, where it reacted with a membrane-impermeant pyrylium to form a fluorescent pyridinium salt ${ }^{63}$ (Figure $31 \mathrm{~A}$ ). Protein synthesis by IVTT has also been achieved inside two-droplet multisomes generated with the phase transfer method $^{74}$ (Figure 31B). Different fluorescent proteins were expressed in each of the two compartments.

\subsubsection{Communication and interfaces with other structures and tissues}

Multisomes or larger droplet networks might be integrated with living tissues. One possibility is to use gap junction-like proteins to bridge the two lipid bilayers that would separate the two structures. Liposomes with bilayerintegrated connexin 43 have been shown to couple to cells expressing the same gap junction protein and to deliver their contents to the cells ${ }^{166}$. By covalently coupling two $\alpha \mathrm{HL}$ pores in an aligned cap-to-cap orientation, a gap junction-like protein has been formed ${ }^{167}$ (Figure 32). The molecule will bridge two liposomes, albeit inefficiently, but is yet to be tested with multisomes or droplet networks. 
Biological signaling is often mediated by water-soluble small molecules that physically cross or bind to cell membranes, primarily through membrane protein receptors ${ }^{168}$. Ultimately, multisomes or larger droplet networks might be equipped with receptors to receive such signals or with the means to release signaling molecules. Interactions have already been demonstrated between lipid vesicles and prokaryotic cells ${ }^{169}$. The vesicles contained an IVTT system that responded to theophylline by expressing $\alpha \mathrm{HL}$, which in turn mediated the release of IPTG. The IPTG then initiated gene expression in a responsive strain of $\mathrm{E}$. coli.

\subsubsection{Compartments with internal structures: synthetic organelles}

It is possible to consider the compartments of multisomes as being analogous to organelles in a cell, rather than as individual cells ${ }^{57,74,170}$. Other types of compartmentalized or nested structures have also been generated, which are explored in this Section. It would be of interest to integrate these structures into printed droplet networks, again with organelles in mind.

Ces and colleagues have proposed that large vesicles be equipped with smaller internal vesicles that would act as synthetic organelles ${ }^{171}$. In the original proposal, the organelles were designed to control gene transcription, but additional functions can be envisaged, notably the ability to produce ATP to power biochemical systems within liposomes or droplets. Such internal liposomes have been generated microfluidically by using the w/o/w double emulsion dewetting method ${ }^{172}$ (see Section 2.5). Single-compartment liposomes formed by this method were used as the inner aqueous phase in a second w/o/w double emulsion, resulting in "vesosomes" with one or more internal compartments. Further, various biochemical systems were encapsulated within these compartments. By using an in vitro transcription mix, RNA production was localized to an inner compartment, thereby mimicking a cell nucleus. Simultaneously, an IVTT mix in the outer aqueous compartment enabled localized protein expression. To better resemble gene expression, it would be necessary to couple the RNA and protein synthesis, which would require the transfer of biopolymers between compartments. Deng and colleagues did demonstrate the selective transfer of small dye molecules between compartments by encapsulating melittin, a pore-forming peptide, in the inner compartment.

Other types of membranes, which offer increased toughness or controlled permeability, can also be used to generate multi-compartment systems. Polymersomes are aqueous compartments surrounded by layers of amphiphilic block copolymers ${ }^{173}$. Polymersomes have been created by microfluidics as w/o/w systems, which after oil removal contained two, three or eight compartments, up to $\sim 0.4 \mathrm{~nL}$ in volume ${ }^{174}$ (Figure 33A, B). In microfluidically-generated concentric multilayer polymersomes, the programmed release of material from different compartments was achieved by constructing each layer from a diblock copolymer with or without a poly(lactic acid) homopolymer additive. The rupture of each layer was then triggered sequentially, based on the differential stability of the layers towards ethanol in 
the medium ${ }^{175}$ (Figure 33C, D). Further, enzymatic reaction cascades have been carried out by entrapping different enzymes within organelle-like polymersomes inside larger polymersomes ${ }^{176}$. Reagents and intermediates diffused through the semipermeable membranes. To further control the transfer of materials between compartments, protein pores might be incorporated into the internal and external bilayers. Membrane proteins have been shown to insert into the block copolymer membranes of singlecompartment polymersomes during IVTT $^{177}$, to form proteopolymersomes. Therefore, IVTT systems might be encapsulated within multi-compartment polymersomes, to allow communication between internal compartments and with the environment. Block copolymer membranes are then a promising means to improve the stability of droplet systems. Indeed, the long-term stability of block copolymer membranes has been demonstrated in Oxford Nanopore's MinION chips.

Millimetre-scale aqueous-core alginate capsules have been generated by simultaneously dripping an inner aqueous solution and an outer, alginatecontaining aqueous solution from two coaxial tubes ${ }^{178}$. The droplets were ejected into a calcium chloride bath where a thin hydrogel membrane was formed when the alginate layer became crosslinked with $\mathrm{Ca}^{2+}$. Shape change of aqueous-core capsules due to the osmotically driven movement of water through the hydrogel membrane has been demonstrated ${ }^{179}$. The long-term stability of these capsules was not specified. Rupture could be induced by the addition of a chelator to sequester the divalent cations. Co-extrusion allowed the formation of multi-compartment capsules with two or three distinct compartments $^{180}$ (Figure 33E-G). Microfluidically generated hydrogel capsules have been used to encapsulate droplet networks ${ }^{56}$ (see Section 2.5).

Multi-vesicle assemblies have been created by establishing various interactions between the vesicle membranes, e.g. biotin-streptavidin ${ }^{181}$, cationic-anionic linker molecules ${ }^{182}$ and DNA hybridization ${ }^{183,}{ }^{184}$. These assembly methods allow little control over the final composition and organization of the assemblies. Furthermore, communication between the individual compartments has not been demonstrated.

Again, the ability to integrate these structures into printed droplet networks would considerably enhance their functionality, allowing the constructions of materials that can be truly considered as synthetic tissues.

\section{Future prospects}

The development of DIBs and droplet networks is far from complete, and offers numerous possibilities for understanding and improvement, both in terms of fundamental science and applications in biotechnology and medicine. Science is often most productive if fundamental studies and applications (Figure 34) proceed in parallel, and this area will be no exception.

While we have noted several excellent individual studies, there has been no systematic examination of the physical chemistry of DIBs and droplet networks. We assume that DIB formation, rather than droplet fusion, occurs 
only after monolayer formation is complete. With DPhPC, monolayer formation takes several minutes for a $\mathrm{nL}$ aqueous droplet in unstirred oil-containing lipid $^{3}$, and in microfluidics systems delay lines have been used to ensure completion of the process before droplets meet ${ }^{83,85}$. The process is quicker when oil-containing lipid flows around the droplet ${ }^{3}$. Monolayers form more rapidly around $\mathrm{pL}$ droplets ${ }^{15}$. With a mixture of DPhPC and PEGylated lipids, bilayers form faster after droplet formation than when DPhPC alone is used ${ }^{16}$. In $3 \mathrm{D}$ printing it is critical that monolayers are formed before contact between droplets, and may limit the printing rate (see Section 2.4). The fragmentary nature of these observations accentuates the need for a more cohesive investigation of DIB formation.

The strength of a DIB (as judged by the contact angle between two droplets) is affected by several variables including the lipid used, the oil, droplet size, the temperature, the internal contents and the transmembrane potential (see Section 1.2). A methodical examination of these factors would provide information that would allow the strength of a DIB between a new droplet combination to be predicted. DIBs exist in a local energy minimum and tend to fuse. Again, means to stabilize DIBs kinetically against fusion would be welcome. Droplets within small networks, multisomes and printed networks will experience forces that differ from those in droplet pairs and need to be examined further for stability towards fusion. The co-assembly, packing and fusion of droplets differing in volume have been hardly considered. Further, the viscoelastic properties of printed droplet networks have not been seriously investigated and the available information is largely anecdotal (see Section 2.4). Elastic properties, time-dependent deformation and permanent damage ${ }^{185}$ are accessible to a variety of modern mechanical and rheological tools ${ }^{186}$, which can measure both ensemble properties and map local variation.

Means to incorporate additional features into DIBs and droplet networks and stabilize the constructs offer many opportunities for improvements. Protein pores, channels and transporters have been incorporated into DIBs and the surface bilayers of multisomes, but the armory is far from exhausted. For example, receptor proteins have not been examined. Methods for the efficient and vectorial incorporation of membrane proteins into DIBs deserve further exploration and generalization. DIBs might be functionalized with entities other than proteins; for example, DNA nanostructures have significant potential for functional pore formation ${ }^{187}$. DIBs and droplet networks can also be modified by the incorporation of chemical and biochemical components into the internal aqueous compartments. However, small molecules can leak out into the surrounding oil or through the surface bilayers of multisomes. To some extent, leakage might be prevented by varying the composition of the oil, although this has not been examined in detail, e.g. fluorocarbons might be tested. Where possible, the best bet is probably to encapsulate charged molecules, which is the strategy of living cells. Biochemical components, such as high concentrations of proteins, can destabilize DIBs. One strategy to prevent bilayer breakdown is to use lipids with modified headgroups, such as the PEGderivatized lipids used by Booth and colleagues ${ }^{16}$. Droplets may be further elaborated by the inclusion of small functionalized lipid vesicles ${ }^{91,171}$ (see Section 3.4.4), which could for example act as energy sources by mimicking 
chloroplasts or mitochondria and therefore must be stabilized towards fusion with DIBs. DIBs and droplet networks can be stabilized by using internal hydrogels or external organogels ${ }^{34,55-57}$ and the numerous possible variations on this theme have been hardly explored. Further, hydrogel shapes can also be used to form networks connected by $\mathrm{DIBs}^{52}$ and this work might be profitably elaborated.

The 3D printers used to make extended droplet networks will doubtless be further improved in the near future. Upgraded printers have been implemented recently with improved software and hardware, temperature control and the ability to eject droplets of viscous solutions (S. Box, S. Ma and L. Zhou, unpublished). The present printers are slow (1 to 2 droplets per second, which might be remedied by using a parallel printer with many delivery nozzles. For instance, ten or twenty nozzles would improve speed by a factor of ten. Such a printer would also be able to position droplets in more elaborate patterns. An exciting possibility is to combine the rapid generation of uniform droplets by microfluidics with a printing head ${ }^{188}$. $4 \mathrm{D}$ printing, in which printed structures fold after printing ${ }^{15}$, also has considerable potential ${ }^{150}$. Co-printing with or around additional components should also be examined seriously. For example, fibers that brace a structure or microtubes that convey fluids through it might be included. The fabrication of large networks in the $\mathrm{cm}$ range might be achieved by assembling $\mathrm{mm}$-scale networks (printed in parallel) with a robotic device, providing speed and additional potential for patterning.

The functional properties of 3D-printed networks might be expanded by several means. Methods for coupling networks to each other or to other structures should be further developed. For example, adhesion in an aqueous medium might be provided through natural protein-protein interactions or, alternatively, artificial means, such as DNA hybridization ${ }^{189}$, might prove useful. Communication and molecular transfer through engineered connections resembling gap junctions is an attractive possibility and initial attempts $^{167}$ deserve further exploration. Internal communication through droplet networks by diffusion ${ }^{62}$, electrical signaling ${ }^{3,15}$ and chemical waves ${ }^{84,}$ 126 has been demonstrated. Opportunities exist for controlling the initiation of signaling and for coupling the propagated signal to a significant output. Shape changes in printed droplet networks were achieved by making use of osmotic water flow through DIBs. The shape changes were slow and irreversible and the requirement for an osmotic gradient limited the additional functionalities that could be incorporated into the networks. Progress in this area would enhance the utility of the networks and eventually lead to mechanical abilities such as the lifting and placing of objects and to motility, for example swimming.

The remote (external) control of 3D-printed networks is a challenging prospect and strictly-controlled light activation of an encapsulated IVTT system has recently been achieved ${ }^{16}$. Additional possibilities, of several, include magnetic or radiofrequency stimulation. The receivers for the stimuli might be printed only in selected droplets or, where it is possible, a stimulus might be applied in a structured manner. On-off switches and orthogonal stimuli are important goals. This area is intensely active. For example, it has been claimed that 
magnetic fields can be used to control neuronal function, through the genetic encoding of natural magnetoreceptors ${ }^{190}$ or the fusion of channel proteins with the paramagnetic protein ferritin ${ }^{191}$, and to induce gene expression, through the use of a heat-inducible gene promoter activated by iron oxide nanoparticles $^{192}$. However, the use of magnetism to control biological systems is controversial and the several suggested mechanisms of activation are viewed with suspicion ${ }^{193}$.

Another useful functionality for droplet networks is uptake, conversion and release. For example, a network might take up a small molecule, which is then converted to a product by an encapsulated enzyme, followed by release into the external medium. While this goal has not yet been approached, release from multisomes has been demonstrated by using $\mathrm{pH}$ - or temperaturesensitive lipids ${ }^{89}$ (Figure 29) and might be employed for drug delivery. Indeed, the efficient encapsulation of drug molecules in robust multi-compartment liposomes (a crude form of multisome, see Section 2.5) has already been demonstrated $^{194}$. This technology, known as DepoFoam ${ }^{\mathrm{TM}}$, has been approved by the US FDA for two clinical formulations, DepoCyt ${ }^{\mathrm{TM}}$, which contains cytarabine an antineoplastic agent, and DepoDur ${ }^{\mathrm{TM}}$, for the sustained release of morphine. Vesosomes (see Section 3.4.4) have also been explored as drug delivery vehicles ${ }^{195}$ and also offer greater stability compared to unilamellar liposomes ${ }^{196}$. Multisomes, which can be formed from droplets of uniform volume and from droplets that differ in content, and which can be patterned by design, offer yet more sophisticated possibilities for drug delivery. For instance, multisomes might be used for binary drug delivery in which the contents of two compartments are mixed at the target site, for example to combine a prodrug and its activator ${ }^{89}$.

Energy generation is also an important goal. Both concentration cells and photogenerated currents, mediated by $b R$, were demonstrated in early work ${ }^{3}$. Most recently, patterned current generation has been established in an array of bR-containing droplets ${ }^{144}$ (see Section 3.2.1), with the long-term goal of implanting such arrays within droplet networks to allow patterned energization. Biofuel cells ${ }^{197}$ are also an interesting possibility, unexplored in this context. Both concentration and biofuel cells would need recharging, a difficult prospect. Another serious challenge is to couple generated electrical current to useful work or to the formation of ATP for use in biochemical transformations.

Finally, living cells can be incorporated into droplet networks. For example, the 3D droplet printer described here has been used with cell-containing "bioinks" to generate structures with multiple cell types patterned in predetermined configurations $^{198}$. These structures, the acellular droplet networks detailed in this review and natural tissues might be integrated ${ }^{167}$ into hybrid systems with potentially important applications in medicine, for example to repair or enhance the properties of damaged organs.

If just some of the improvements and developments described in this section can be introduced, the prospects for applications of 3D-printed droplet networks in biotechnology and medicine will be greatly enhanced. In 
biotechnology, the development of microscale soft robots is a fascinating prospect. In medicine, near-term applications include drug delivery and longerterm prospects include materials that can repair or enhance failing organs.

\section{Conflicts of Interest}

Hagan Bayley is the Founder of, a Director of, a share-holder in and a consultant for OxSyBio, a company engaged in the development of printed tissues and tissue-like materials. Work in the Bayley laboratory at the University of Oxford is supported in part by OxSyBio. Hagan Bayley is also the Founder of, a consultant for and a share-holder of Oxford Nanopore Technologies, a company engaged in the development of nanopore sensing and sequencing technologies. 


\section{Abbreviations}

$\alpha \mathrm{HL}$

Amplex Red

as-BLM

BLM

bR

BZ

CMC

ClyA -

DDAB

DDM

DHB

DIB

DOPC

DOPE

DPhPC

DPPG

DPPE-mPEG2000

DREADD

dsDNA

DSPC

EWOD

GUI

h-BLM

HRP

IVTT

IPTG

$\mathrm{KcV}$

lacY

LA-DNA

MscL

$\mathrm{Nd}$

PEG

PDMS

PMMA

pc-BLM

s-BLM

SEBS

ssDNA

TIRF

TRPM8

w/o/w
Staphylococcal $\alpha$-hemolysin

(N-acetyl-3,7-dihydroxyphenoxazine)

Air-stable black lipid membranes

Black lipid membranes

Bacteriorhodopsin

Belousov Zhabotinsky oscillating reaction

Critical micelle concentration

Cytolysin A from Escheria coli

Dimethyldioctadecylammonium bromide

n-Dodecyl- $\beta$-maltoside

Bilayer at a droplet/hydrogel interface

Droplet interface bilayer

1,2-dioleoyl-sn-glycero-3-phosphocholine)

1,2-dioleoyl-sn-glycero-3-phosphoethanolamine)

Diphytanoyl-sn-glycerol-3-phosphocholine

Dipalmitoyl-sn-glycero-3-[phospho-rac-(1-glycerol)]

1,2-dipalmitoyl-sn-glycero-3-phosphoethanolamine-N[methoxy(polyethylene glycol)-2000]

Designer Receptor Exclusively Activated by Designer

Drugs

double stranded DNA

1,2-distearoyl-sn-glycero-3-phosphocholine

Electrowetting on dielectric

Graphical user interface

Hybrid black lipid membranes

Horse-radish peroxidase

In vitro transcription and translation

Isopropyl $\beta$-D-1-thiogalactopyranoside

Potassium ion channel

A lactose transporter

Light-activated DNA

Large Conductance Mechanosensitive Ion Channel

Neodynium

Poly(ethylene glycol)

Poly(dimethyl siloxane)

Poly(methyl methacrylate)

Polymer-cushioned black lipid membranes

supported black lipid membranes

poly[styrene-b-(ethylene-co-butylene)-b-styrene

single stranded DNA

Total internal reflection fluorescence

Transient receptor potential cation channel subfamily $M$ member 8

Water in oil in water 


\section{References}

1. H. Bayley, B. Cronin, A. Heron, M. A. Holden, W. L. Hwang, R. Syeda, J. Thompson and M. Wallace, Mol Biosyst, 2008, 4, 1191-1208.

2. D. Needham, Nat Nanotechnol, 2011, 6, 761-762.

3. M. A. Holden, D. Needham and H. Bayley, J Am Chem Soc, 2007, 129, 8650-8655.

4. $\quad$ L. M. Tsofina, E. A. Liberman and A. V. Babakov, Nature, 1966, 212, 681683.

5. K. Funakoshi, H. Suzuki and S. Takeuchi, Anal Chem, 2006, 78, 81698174.

6. N. Malmstadt, M. A. Nash, R. F. Purnell and J. J. Schmidt, Nano Lett, 2006, 6, 1961-1965.

7. A. J. Heron, J. R. Thompson, A. E. Mason and M. I. Wallace, J Am Chem Soc, 2007, 129, 16042-16047.

8. V. M. Aguilella and A. Alcaraz, Nat Nanotechnol, 2009, 4, 403-404.

9. S. A. Sarles and D. J. Leo, Smart Mater Struct, 2011, 20.

10. M. Zagnoni, Lab Chip, 2012, 12, 1026-1039.

11. S. Leptihn, O. K. Castell, B. Cronin, E. H. Lee, L. C. M. Gross, D. P. Marshall, J. R. Thompson, M. Holden and M. I. Wallace, Nat Protoc, 2013, 8, 1048-1057.

12. M. A. Holden, Methods Cell Biol, 2015, 128, 201-222.

13. N. E. Barlow, E. Smpokou, M. S. Friddin, R. Macey, I. R. Gould, C. Turnbull, A. J. Flemming, N. J. Brooks, O. Ces and L. M. C. Barter, Biomicrofluidics, 2017, 11.

14. G. J. Taylor and S. A. Sarles, Langmuir, 2015, 31, 325-337.

15. G. Villar, A. D. Graham and H. Bayley, Science, 2013, 340, 48-52.

16. M. J. Booth, V. R. Schild, A. D. Graham, S. N. Olof and H. Bayley, Sci Adv, 2016, 2, e1600056.

17. C. A. Helm, J. N. Israelachvili and P. M. McGuiggan, Biochemistry, 1992, 31, 1794-1805.

18. P. Poulin and J. Bibette, Langmuir, 1998, 14, 6341-6343.

19. A. R. Thiam, N. Bremond and J. Bibette, Phys Rev Lett, 2011, 107, 068301.

20. A. R. Thiam, N. Bremond and J. Bibette, Langmuir, 2012, 28, 6291-6298.

21. G. J. Taylor, G. A. Venkatesan, C. P. Collier and S. A. Sarles, Soft Matter, 2015, 11, 7592-7605.

22. N. Tamaddoni, G. Taylor, T. Hepburn, S. Michael Kilbey and S. A. Sarles, Soft Matter, 2016, 12, 5096-5109.

23. M. Yanagisawa, T. Yoshida, M. Furuta, S. Nakata and M. Tokita, Soft Matter, 2013, 9, 5891-5897.

24. R. Syeda, M. A. Holden, W. L. Hwang and H. Bayley, J Am Chem Soc, 2008, 130, 15543-15548.

25. S. Aghdaei, M. E. Sandison, M. Zagnoni, N. G. Green and H. Morgan, Lab Chip, 2008, 8, 1617-1620.

26. A. Fischer, M. A. Holden, B. L. Pentelute and R. J. Collier, Proc Natl Acad Sci U S A, 2011, 108, 16577-16581.

27. J. B. Boreyko, P. Mruetusatorn, S. A. Sarles, S. T. Retterer and C. P. Collier, J Am Chem Soc, 2013, 135, 5545-5548. 
28. P. Mruetusatorn, J. B. Boreyko, G. A. Venkatesan, S. A. Sarles, D. G. Hayes and C. P. Collier, Soft Matter, 2014, 10, 2530-2538.

29. J. B. Boreyko, G. Polizos, P. G. Datskos, S. A. Sarles and C. P. Collier, Proc Natl Acad Sci U S A, 2014, 111, 7588-7593.

30. P. Mruetusatorn, G. Polizos, P. G. Datskos, G. Taylor, S. A. Sarles, J. B. Boreyko, D. G. Hayes and C. P. Collier, Langmuir, 2015, 31, 4224-4231.

31. W. L. Hwang, M. A. Holden, S. White and H. Bayley, J Am Chem Soc, 2007, 129, 11854-11864.

32. L. C. Gross, A. J. Heron, S. C. Baca and M. I. Wallace, Langmuir, 2011, 27, 14335-14342.

33. S. A. Sarles and D. J. Leo, Anal Chem, 2010, 82, 959-966.

34. S. A. Sarles, L. J. Stiltner, C. B. Williams and D. J. Leo, ACS Appl Mater Interfaces, 2010, 2, 3654-3663.

35. S. A. Sarles, J. D. W. Madden and D. J. Leo, Soft Matter, 2011, 7, 46444653.

36. S. Punnamaraju and A. J. Steckl, Langmuir, 2011, 27, 618-626.

37. A. J. Heron, J. R. Thompson, B. Cronin, H. Bayley and M. I. Wallace, J Am Chem Soc, 2009, 131, 1652-1653.

38. E. E. Weatherill and M. I. Wallace, J Mol Biol, 2015, 427, 146-157.

39. W. L. Hwang, M. Chen, B. Cronin, M. A. Holden and H. Bayley, J Am Chem Soc, 2008, 130, 5878-5879.

40. G. A. Venkatesan, J. Lee, A. B. Farimani, M. Heiranian, C. P. Collier, N. R. Aluru and S. A. Sarles, Langmuir, 2015, 31, 12883-12893.

41. P. F. Devaux, Biochemistry, 1991, 30, 1163-1173.

42. M. A. Czekalska, T. S. Kaminski, S. Jakiela, K. Tanuj Sapra, H. Bayley and P. Garstecki, Lab Chip, 2015, 15, 541-548.

43. J. Huang, M. Lein, C. Gunderson and M. A. Holden, J Am Chem Soc, 2011, 133, 15818-15821.

44. M. Zagnoni, M. E. Sandison, P. Marius and H. Morgan, Anal Bioanal Chem, 2009, 393, 1601-1605.

45. S. A. Portonovo and J. Schmidt, Biomed Microdevices, 2012, 14, 187-191.

46. A. M. El-Arabi, C. S. Salazar and J. J. Schmidt, Lab Chip, 2012, 12, 24092413.

47. R. Kawano, Y. Tsuji, K. Sato, T. Osaki, K. Kamiya, M. Hirano, T. Ide, N. Miki and S. Takeuchi, Sci Rep, 2013, 3, 1995.

48. L. Kong, A. Almond, H. Bayley and B. G. Davis, Nat Chem, 2016, 8, 461469.

49. M. Lein, J. Huang and M. A. Holden, Lab Chip, 2013, 13, 2749-2753.

50. Y. Tsuji, R. Kawano, T. Osaki, K. Kamiya, N. Miki and S. Takeuchi, Lab Chip, 2013, 13, 1476-1481.

51. S. A. Sarles and D. J. Leo, Lab Chip, 2010, 10, 710-717.

52. K. T. Sapra and H. Bayley, Sci Rep, 2012, 2, 848.

53. S. A. Acharya, A. Portman, C. S. Salazar and J. J. Schmidt, Sci Rep, 2013, 3, 3139.

54. M. A. Creasy and D. J. Leo, Smart Materials \& Structures, 2010, 19.

55. G. A. Venkatesan and S. A. Sarles, Lab Chip, 2016, 16, 2116-2125.

56. D. K. Baxani, A. J. Morgan, W. D. Jamieson, C. J. Allender, D. A. Barrow and O. K. Castell, Angew Chem Int Ed Engl, 2016, 55, 14240-14245. 
57. M. Bayoumi, H. Bayley, G. Maglia and K. T. Sapra, Sci Rep, 2017, In Press.

58. S. H. Jung, S. Choi, Y. R. Kim and T. J. Jeon, Bioprocess Biosyst Eng, 2012, 35, 241-246.

59. Y. Bai, X. He, D. Liu, S. N. Patil, D. Bratton, A. Huebner, F. Hollfelder, C. Abell and W. T. Huck, Lab Chip, 2010, 10, 1281-1285.

60. C. E. Stanley, K. S. Elvira, X. Z. Niu, A. D. Gee, O. Ces, J. B. Edel and A. J. Demello, Chem Commun (Camb), 2010, 46, 1620-1622.

61. Y. Elani, A. J. deMello, X. Niu and O. Ces, Lab Chip, 2012, 12, 3514-3520.

62. P. Carreras, Y. Elani, R. V. Law, N. J. Brooks, J. M. Seddon and O. Ces, Biomicrofluidics, 2015, 9, 064121.

63. Y. Elani, X. C. Solvas, J. B. Edel, R. V. Law and O. Ces, Chem Commun (Camb), 2016, 52, 5961-5964.

64. E. Walsh, A. Feuerborn and P. R. Cook, Sci Rep, 2016, 6, 34355.

65. J. L. Poulos, W. C. Nelson, T. J. Jeon, C. J. Kim and J. J. Schmidt, Applied Physics Letters, 2009, 95.

66. S. S. Dixit, H. Kim, A. Vasilyev, A. Eid and G. W. Faris, Langmuir, 2010, 26, 6193-6200.

67. M. S. Friddin, G. Bolognesi, Y. Elani, N. J. Brooks, R. V. Law, J. M. Seddon, M. A. Neil and O. Ces, Soft Matter, 2016, 12, 7731-7734.

68. T. Wauer, H. Gerlach, S. Mantri, J. Hill, H. Bayley and K. T. Sapra, ACS Nano, 2014, 8, 771-779.

69. S. Leptihn, J. R. Thompson, J. C. Ellory, S. J. Tucker and M. I. Wallace, J Am Chem Soc, 2011, 133, 9370-9375.

70. N. Rojko, B. Cronin, J. S. Danial, M. A. Baker, G. Anderluh and M. I. Wallace, Biophys J, 2014, 106, 1630-1637.

71. M. S. Friddin, N. P. Smithers, M. Beaugrand, I. Marcotte, P. T. Williamson, H. Morgan and M. R. de Planque, Analyst, 2013, 138, 72947298.

72. H. E. Findlay, N. J. Harris and P. J. Booth, Sci Rep, 2016, 6, 39349.

73. M. S. Friddin, H. Morgan and M. R. de Planque, Biomicrofluidics, 2013, 7, 14108.

74. Y. Elani, R. V. Law and O. Ces, Phys Chem Chem Phys, 2015, 17, 1553415537.

75. Y. Tsuji, R. Kawano, T. Osaki, K. Kamiya, N. Miki and S. Takeuchi, Anal Chem, 2013, 85, 10913-10919.

76. D. J. Collins, B. Morahan, J. Garcia-Bustos, C. Doerig, M. Plebanski and A. Neild, Nat Commun, 2015, 6, 8686.

77. T. Nisisako, S. Okushima and T. Torii, Soft Matter, 2005, 1, 23-27.

78. L. Y. Chu, A. S. Utada, R. K. Shah, J. W. Kim and D. A. Weitz, Angew Chem Int Ed Engl, 2007, 46, 8970-8974.

79. M. Zagnoni and J. M. Cooper, Lab Chip, 2010, 10, 3069-3073.

80. B. Schlicht and M. Zagnoni, Sci Rep, 2015, 5, 9951.

81. R. Dangla, S. Lee and C. N. Baroud, Phys Rev Lett, 2011, 107, 124501.

82. E. Fradet, C. McDougall, P. Abbyad, R. Dangla, D. McGloin and C. N. Baroud, Lab Chip, 2011, 11, 4228-4234.

83. P. H. King, G. Jones, H. Morgan, M. R. de Planque and K. P. Zauner, Lab Chip, 2014, 14, 722-729. 
84. G. Jones, P. H. King, H. Morgan, M. R. de Planque and K. P. Zauner, Artif Life, 2015, 21, 195-204.

85. P. Carreras, R. V. Law, N. Brooks, J. M. Seddon and O. Ces, Biomicrofluidics, 2014, 8, 054113.

86. E. J. Challita, J. S. Najem, E. C. Freeman and D. J. Leo, Proc. SPIE 10167, Nanosensors, Biosensors, Info-Tech Sensors and 3D Systems, 2017, 10167.

87. I. Levental, P. C. Georges and P. A. Janmey, Soft Matter, 2007, 3, 299-306.

88. S. Kim, M. S. Turker, E. Y. Chi, S. Sela and G. M. Martin, Biochim Biophys Acta, 1983, 728, 339-348.

89. G. Villar, A. J. Heron and H. Bayley, Nat Nanotechnol, 2011, 6, 803-808.

90. Y. Elani, A. Gee, R. V. Law and O. Ces, Chemical Science, 2013, 4, 33323338.

91. N. N. Deng, M. Yelleswarapu and W. T. Huck, J Am Chem Soc, 2016, 138, 7584-7591.

92. R. Kawano, Y. Tsuji, K. Kamiya, T. Kodama, T. Osaki, N. Miki and S. Takeuchi, PLoS One, 2014, 9, e102427.

93. N. Tamaddoni, E. C. Freeman and S. A. Sarles, Smart Materials $\mathcal{E}$ Structures, 2015, 24.

94. N. Tamaddoni and S. A. Sarles, Bioinspiration E Biomimetics, 2016, 11.

95. J. S. Najem, M. D. Dunlap, I. D. Rowe, E. C. Freeman, J. W. Grant, S. Sukharev and D. J. Leo, Sci Rep, 2015, 5, 13726.

96. J. S. Najem, M. D. Dunlap, A. Yasmann, E. C. Freeman, J. W. Grant, S. Sukharev and D. J. Leo, J Vis Exp, 2015.

97. E. C. Freeman, J. S. Najem, S. Sukharev, M. K. Philen and D. J. Leo, Soft Matter, 2016, 12, 3021-3031.

98. H. M. Barriga, P. Booth, S. Haylock, R. Bazin, R. H. Templer and O. Ces, J R Soc Interface, 2014, 11, 20140404.

99. M. Lein, B. M. deRonde, F. Sgolastra, G. N. Tew and M. A. Holden, Biochim Biophys Acta, 2015, 1848, 2980-2984.

100. A. M. Waszkielewicz, A. Gunia, N. Szkaradek, K. Sloczynska, S. Krupinska and H. Marona, Curr Med Chem, 2013, 20, 1241-1285.

101. G. V. Petkov, Nat Rev Urol, 2011, 9, 30-40.

102. L. Chen, B. Tuo and H. Dong, Nutrients, 2016, 8.

103. Z. Varga, P. Hajdu and G. Panyi, Immunol Lett, 2010, 130, 19-25.

104. A. E. Boyd, 3rd, J Cell Biochem, 1992, 48, 235-241.

105. D. A. Pereira and J. A. Williams, Br J Pharmacol, 2007, 152, 53-61.

106. Y. Sun, S. Selvaraj, A. Varma, S. Derry, A. E. Sahmoun and B. B. Singh, J Biol Chem, 2013, 288, 255-263.

107. C. Wood, C. Williams and G. J. Waldron, Drug Discov Today, 2004, 9, 434-441.

108. T. J. Dale, C. Townsend, E. C. Hollands and D. J. Trezise, Mol Biosyst, 2007, 3, 714-722.

109. M. X. Chen, K. Gatfield, E. Ward, D. Downie, H. F. Sneddon, S. Walsh, A. J. Powell, D. Laine, M. Carr and D. Trezise, J Biomol Screen, 2015, 20, 242-253.

110. M. S. Khan, N. S. Dosoky and J. D. Williams, Int J Mol Sci, 2013, 14, 21561-21597. 
111. G. Baaken, N. Ankri, A. K. Schuler, J. Ruhe and J. C. Behrends, ACS Nano, 2011, 5, 8080-8088.

112. T. Thapliyal, J. L. Poulos and J. J. Schmidt, Biosens Bioelectron, 2011, 26, 2651-2654.

113. N. E. Barlow, G. Bolognesi, A. J. Flemming, N. J. Brooks, L. M. Barter and O. Ces, Lab Chip, 2016, 16, 4653-4657.

114. J. L. Poulos, T. J. Jeon, R. Damoiseaux, E. J. Gillespie, K. A. Bradley and J. J. Schmidt, Biosens Bioelectron, 2009, 24, 1806-1810.

115. J. L. Poulos, S. A. Portonovo, H. Bang and J. J. Schmidt, J Phys Condens Matter, 2010, 22, 454105.

116. O. K. Castell, J. Berridge and M. I. Wallace, Angew Chem Int Ed Engl, 2012, 51, 3134-3138.

117. T. Tonooka, K. Sato, T. Osaki, R. Kawano and S. Takeuchi, Small, 2014, 10, 3275-3282.

118. A. Kleefen, D. Pedone, C. Grunwald, R. Wei, M. Firnkes, G. Abstreiter, U. Rant and R. Tampe, Nano Lett, 2010, 10, 5080-5087.

119. M. Urban, A. Kleefen, N. Mukherjee, P. Seelheim, B. Windschiegl, M. Vor der Bruggen, A. Kocer and R. Tampe, Nano Lett, 2014, 14, 16741680.

120. S. Huang, M. Romero-Ruiz, O. K. Castell, H. Bayley and M. I. Wallace, Nat Nanotechnol, 2015, 10, 986-991.

121. T. Osaki, H. Suzuki, B. Le Pioufle and S. Takeuchi, Anal Chem, 2009, 81, 9866-9870.

122. S. Ota, H. Suzuki and S. Takeuchi, Lab Chip, 2011, 11, 2485-2487.

123. B. Lu, G. Kocharyan and J. J. Schmidt, Biotechnol J, 2014, 9, 446-451.

124. WO2014064443 A2, 2014.

125. Y. Elani, R. V. Law and O. Ces, Nat Commun, 2014, 5, 5305.

126. S. Thutupalli and S. Herminghaus, Eur Phys J E Soft Matter, 2013, 36, 91.

127. J. Guzowski, K. Gizynski, J. Gorecki and P. Garstecki, Lab Chip, 2016, 16, 764-772.

128. M. Szabo and M. I. Wallace, Biochim Biophys Acta, 2016, 1858, 613-617.

129. J. R. Thompson, A. J. Heron, Y. Santoso and M. I. Wallace, Nano Lett, 2007, 7, 3875-3878.

130. J. T. Sengel and M. I. Wallace, Proc Natl Acad Sci U S A, 2016, 113, 52815286.

131. L. C. Gross, O. K. Castell and M. I. Wallace, Nano Lett, 2011, 11, 33243328.

132. J. R. Thompson, B. Cronin, H. Bayley and M. I. Wallace, Biophys J, 2011, 101, 2679-2683.

133. M. A. Baker, N. Rojko, B. Cronin, G. Anderluh and M. I. Wallace, Chembiochem, 2014, 15, 2139-2145.

134. L. M. Harriss, B. Cronin, J. R. Thompson and M. I. Wallace, J Am Chem Soc, 2011, 133, 14507-14509.

135. J. S. Danial, B. Cronin, C. Mallik and M. I. Walllace, Soft Matter, 2017, 13, 1788-1793.

136. G. de Wit, J. S. Danial, P. Kukura and M. I. Wallace, Proc Natl Acad Sci U $S$ A, 2015, 112, 12299-12303. 
137. D. T. Simon, K. C. Larsson, D. Nilsson, G. Burstrom, D. Galter, M.

Berggren and A. Richter-Dahlfors, Biosens Bioelectron, 2015, 71, 359-364.

138. G. Dagnelie, Curr Opin Neurol, 2012, 25, 67-75.

139. K. Manoli, M. Magliulo, M. Y. Mulla, M. Singh, L. Sabbatini, G. Palazzo and L. Torsi, Angew Chem Int Ed Engl, 2015, 54, 12562-12576.

140. J. Liu, T. M. Fu, Z. Cheng, G. Hong, T. Zhou, L. Jin, M. Duvvuri, Z. Jiang, P. Kruskal, C. Xie, Z. Suo, Y. Fang and C. M. Lieber, Nat Nanotechnol, 2015, 10, 629-636.

141. P. J. Rousche and R. A. Normann, J Neurosci Methods, 1998, 82, 1-15.

142. G. Maglia, A. J. Heron, W. L. Hwang, M. A. Holden, E. Mikhailova, Q. Li, S. Cheley and H. Bayley, Nat Nanotechnol, 2009, 4, 437-440.

143. J. Xu, F. J. Sigworth and D. A. LaVan, Adv Mater, 2010, 22, 120-127.

144. V. R. Schild, M. J. Booth, S. J. Box, K. R. Mahendran, S. N. Olof and H. Bayley, Sci Rep, 2017, In Press.

145. H. Yasuga, R. Kawano, M. Takinoue, Y. Tsuji, T. Osaki, K. Kamiya, N. Miki and S. Takeuchi, 17th International Conference on Miniaturized Systems for Chemistry and Life Sciences, MicroTAS 2013, 2013, 3, 19141916.

146. K. Inoue, R. Kawano, H. Yasuga, M. Takinoue, T. Osaki, K. Kamiya, N. Miki and S. Takeuchi, 17th International Conference on Miniaturized Systems for Chemistry and Life Sciences, MicroTAS 2013, 2013, 3, 18811883.

147. H. Yasuga, R. Kawano, M. Takinoue, Y. Tsuji, T. Osaki, K. Kamiya, N. Miki and S. Takeuchi, PLoS One, 2016, 11, e0149667.

148. J. Gorecki, K. Gizynski, J. Guzowski, J. N. Gorecka, P. Garstecki, G. Gruenert and P. Dittrich, Philos Trans A Math Phys Eng Sci, 2015, 373.

149. N. J. Russell, J Physiol, 1980, 298, 347-360.

150. Y. C. Li, Y. S. Zhang, A. Akpek, S. R. Shin and A. Khademhosseini, Biofabrication, 2016, 9, 012001.

151. S. S. Dixit, A. Pincus, B. Guo and G. W. Faris, Langmuir, 2012, 28, 74427451.

152. J. M. Skotheim and L. Mahadevan, Science, 2005, 308, 1308-1310.

153. Y. Forterre, J. M. Skotheim, J. Dumais and L. Mahadevan, Nature, 2005, 433, 421-425.

154. T. Zhang, D. Wan, J. M. Schwarz and M. J. Bowick, Phys Rev Lett, 2016, 116, 108301.

155. A. Gutowska, J. S. Bark, I. C. Kwon, Y. H. Bae, Y. Cha and S. W. Kim, J Control Release, 1997, 48, 141-148.

156. E. Sinibaldi, A. Argiolas, G. L. Puleo and B. Mazzolai, PLoS One, 2014, 9.

157. X. Chen, L. Mahadevan, A. Driks and O. Sahin, Nat Nanotechnol, 2014, 9, 137-141.

158. Y. Shimizu, T. Kanamori and T. Ueda, Methods, 2005, 36, 299-304.

159. J. Olejnik, S. Sonar, E. Krzymanska-Olejnik and K. J. Rothschild, Proc Natl Acad Sci U S A, 1995, 92, 7590-7594.

160. M. Howarth, D. J. Chinnapen, K. Gerrow, P. C. Dorrestein, M. R. Grandy, N. L. Kelleher, A. El-Husseini and A. Y. Ting, Nat Methods, 2006, 3, 267-273. 
161. S. Loudwig, H. Bayley, L. Peng, M. Maurice Goeldner, J. S. Condeelis and L. D. S., in Dynamic Studies in Biology: Phototriggers, Photoswitches and Caged Biomolecules, eds. M. Goeldner and R. S. Givens, Wiley-VCH Verlag, Weinheim, 2005, pp. 253-304.

162. T. M. Allen and P. R. Cullis, Adv Drug Deliv Rev, 2013, 65, 36-48.

163. D. J. Urban and B. L. Roth, Annu Rev Pharmacol Toxicol, 2015, 55, 399417.

164. C. L. Chan, G. Bolognesi, A. Bhandarkar, M. S. Friddin, N. J. Brooks, J. M. Seddon, R. V. Law, L. M. C. Barter and O. Ces, Lab Chip, 2016, 16, 4621-4627.

165. S. Punnamaraju, H. You and A. J. Steckl, Langmuir, 2012, 28, 7657-7664.

166. M. Kaneda, S. M. Nomura, S. Ichinose, S. Kondo, K. Nakahama, K. Akiyoshi and I. Morita, Biomaterials, 2009, 30, 3971-3977.

167. S. Mantri, K. T. Sapra, S. Cheley, T. H. Sharp and H. Bayley, Nat Commun, 2013, 4.

168. W. H. Cho and R. V. Stahelin, Annu Rev Biophys Biomol Struct, 2005, 34, 119-151.

169. R. Lentini, S. P. Santero, F. Chizzolini, D. Cecchi, J. Fontana, M. Marchioretto, C. Del Bianco, J. L. Terrell, A. C. Spencer, L. Martini, M. Forlin, M. Assfalg, M. Dalla Serra, W. E. Bentley and S. S. Mansy, Nat Commun, 2014, 5, 4012.

170. Y. Elani, Biochem Soc Trans, 2016, 44, 723-730.

171. D. Miller, P. J. Booth, J. M. Seddon, R. H. Templer, R. V. Law, R. Woscholski, O. Ces and L. M. C. Barter, J R Soc Interface, 2013, 10.

172. N. N. Deng, M. Yelleswarapu, L. Zheng and W. T. Huck, J Am Chem Soc, 2017, 139, 587-590.

173. B. M. Discher, Y. Y. Won, D. S. Ege, J. C. M. Lee, F. S. Bates, D. E. Discher and D. A. Hammer, Science, 1999, 284, 1143-1146.

174. H. C. Shum, Y. J. Zhao, S. H. Kim and D. A. Weitz, Angew Chem Int Ed Engl, 2011, 50, 1648-1651.

175. S. H. Kim, H. C. Shum, J. W. Kim, J. C. Cho and D. A. Weitz, J Am Chem Soc, 2011, 133, 15165-15171.

176. R. J. Peters, M. Marguet, S. Marais, M. W. Fraaije, J. C. van Hest and S. Lecommandoux, Angew Chem Int Ed Engl, 2014, 53, 146-150.

177. S. May, M. Andreasson-Ochsner, Z. Fu, Y. X. Low, D. Tan, H. P. de Hoog, S. Ritz, M. Nallani and E. K. Sinner, Angew Chem Int Ed Engl, 2013, 52, 749-753.

178. N. Bremond, E. Santanach-Carreras, L. Y. Chu and J. Bibette, Soft Matter, 2010, 6, 6207-6207.

179. L. Rolland, E. Santanach-Carreras, T. Delmas, J. Bibette and N. Bremond, Soft Matter, 2014, 10, 9668-9674.

180. F. He, W. Wang, X. H. He, X. L. Yang, M. Li, R. Xie, X. J. Ju, Z. Liu and L. Y. Chu, ACS Appl Mater Interfaces, 2016, 8, 8743-8754.

181. S. A. Walker, M. T. Kennedy and J. A. Zasadzinski, Nature, 1997, 387, 61-64.

182. P. Carrara, P. Stano and P. L. Luisi, Chembiochem, 2012, 13, 1497-1502.

183. M. Hadorn, E. Boenzli, K. T. Sorensen, D. De Lucrezia, M. M. Hanczyc and T. Yomo, Langmuir, 2013, 29, 15309-15319. 
184. Y. H. Chan, P. Lenz and S. G. Boxer, Proc Natl Acad Sci U S A, 2007, 104, 18913-18918.

185. M. Gralka and K. Kroy, Biochim Biophys Acta, 2015, 1853, 3025-3037.

186. P. C. Nalam, N. N. Gosvami, M. A. Caporizzo, R. J. Composto and R. W. Carpick, Soft Matter, 2015, 11, 8165-8178.

187. J. R. Burns, A. Seifert, N. Fertig and S. Howorka, Nat Nanotechnol, 2016, 11, 152-156.

188. S. Ma, N. Mukhkerjee, E. Mikhailova and H. Bayley, Adv Biosys, 2017, 1700075.

189. M. E. Todhunter, N. Y. Jee, A. J. Hughes, M. C. Coyle, A. Cerchiari, J. Farlow, J. C. Garbe, M. A. LaBarge, T. A. Desai and Z. J. Gartner, Nat Methods, 2015, 12, 975-981.

190. X. Long, J. Ye, D. Zhao and S. J. Zhang, Sci Bull (Beijing), 2015, 60, 21072119.

191. M. A. Wheeler, C. J. Smith, M. Ottolini, B. S. Barker, A. M. Purohit, R. M. Grippo, R. P. Gaykema, A. J. Spano, M. P. Beenhakker, S. Kucenas, M. K. Patel, C. D. Deppmann and A. D. Guler, Nat Neurosci, 2016, 19, 756-761.

192. V. Ortner, C. Kaspar, C. Halter, L. Tollner, O. Mykhaylyk, J. Walzer, W. H. Gunzburg, J. A. Dangerfield, C. Hohenadl and T. Czerny, J Control Release, 2012, 158, 424-432.

193. M. Meister, Elife, 2016, 5.

194. M. S. Angst and D. R. Drover, Clin Pharmacokinet, 2006, 45, 1153-1176.

195. B. Wong, C. Boyer, C. Steinbeck, D. Peters, J. Schmidt, R. van Zanten, B. Chmelka and J. A. Zasadzinski, Adv Mater, 2011, 23, 2320-2325.

196. C. Boyer and J. A. Zasadzinski, ACS Nano, 2007, 1, 176-182.

197. Z. Zhu, T. Kin Tam, F. Sun, C. You and Y. H. Percival Zhang, Nat Commun, 2014, 5, 3026.

198. A. D. Graham, S. N. Olof, M. J. Burke, J. P. K. Armstrong, E. A. Mikhailova, J. G. Nicholson, S. J. Box, F. G. Szele, A. P. Perriman and H. Bayley, Sci Rep, 2017, In Press. 

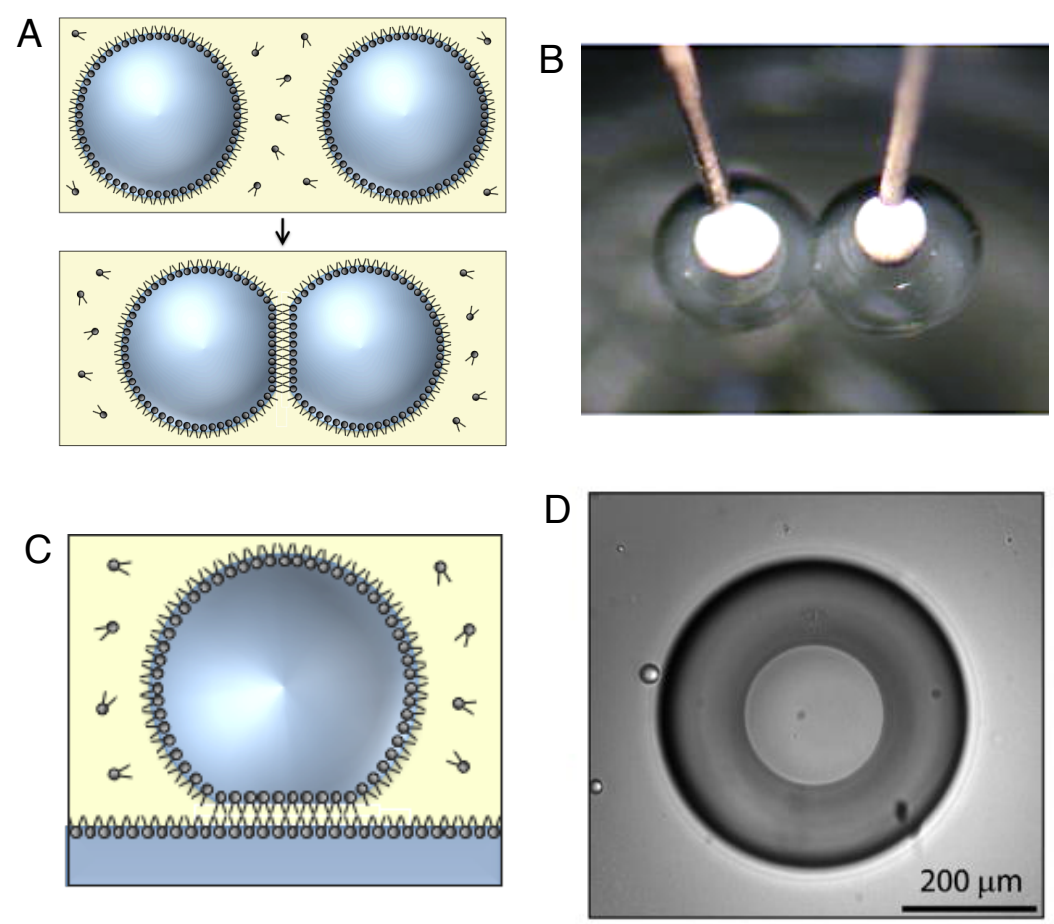

Figure 1. Formation of a droplet interface bilayer (DIB) and a droplet hydrogel bilayer (DHB). (A) Two droplets submerged in a lipid-containing oil, become encapsulated in lipid monolayers. When they are brought together, a kinetically stable lipid bilayer is formed at the interface. (B) A DIB formed from two droplets, each containing an agarose-coated $\mathrm{Ag} / \mathrm{AgCl}$ electrode ${ }^{3}$. (C) $\mathrm{A}$ single droplet and a hydrogel surface, pre-incubated in a lipid-containing oil, form a kinetically stable lipid bilayer (DHB) at their interface. (D) Top view of a DHB. The outer circle corresponds to the droplet, while the inner circle borders the bilayer at the droplet-hydrogel interface ${ }^{7}$. 


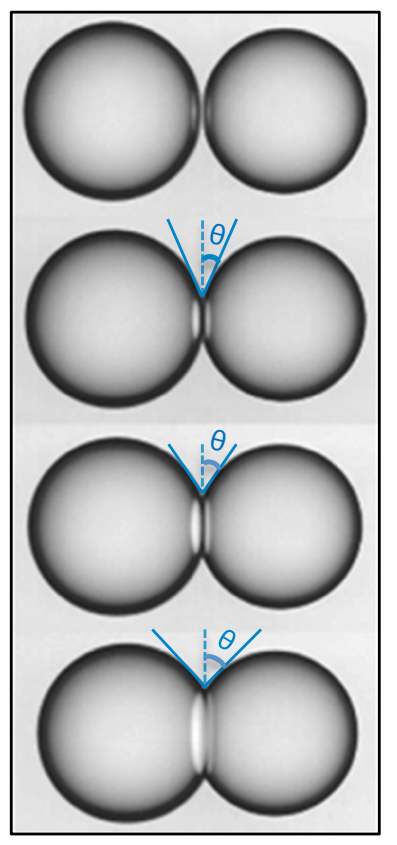

Figure 2. The contact angle is related to the fractional area of a DIB. As the bilayer area increases, the contact angle $(\theta)$ also increases ${ }^{20}$. 


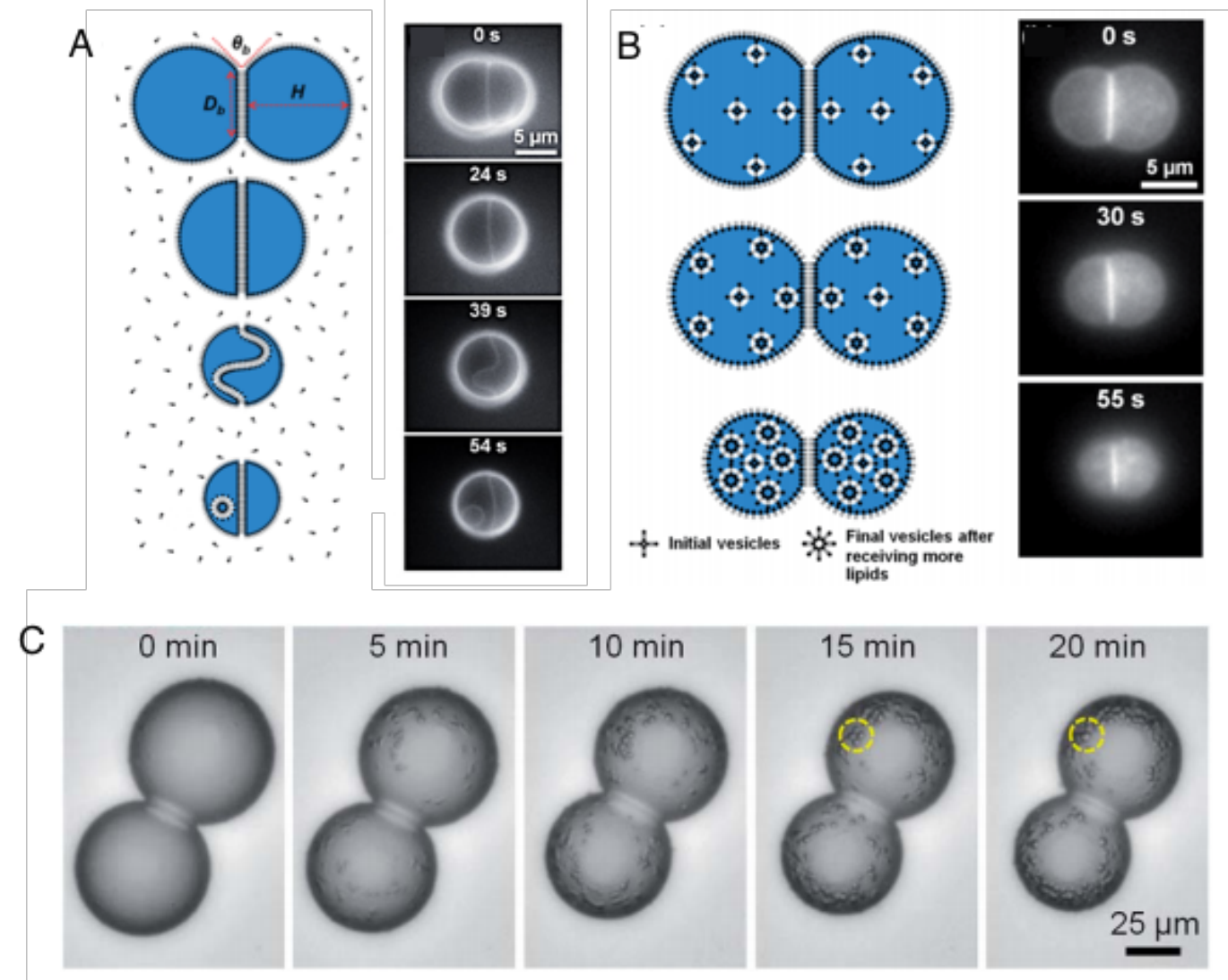

Figure 3. Water loss and droplet shrinkage in droplet pairs ${ }^{28}$. (A) Dehydration of a fL-size droplet pair, with lipid in the external oil. Lipids transferred from the monolayers to the bilayer. The expanded DIB buckled and eventually blebbed to form internal vesicles. (B) When the lipid-in technique (See Section 2.1) was employed, dehydration caused the lipids to transfer from the monolayers and the DIB to internal vesicles, resulting in the uniform shrinkage of the droplets. (C) Alternatively, when larger droplets were dehydrated, in the presence of lipid micelles in the oil, water from the droplets transferred to the hydrophilic interiors of the micelles to form satellite droplets, which grew over time. 

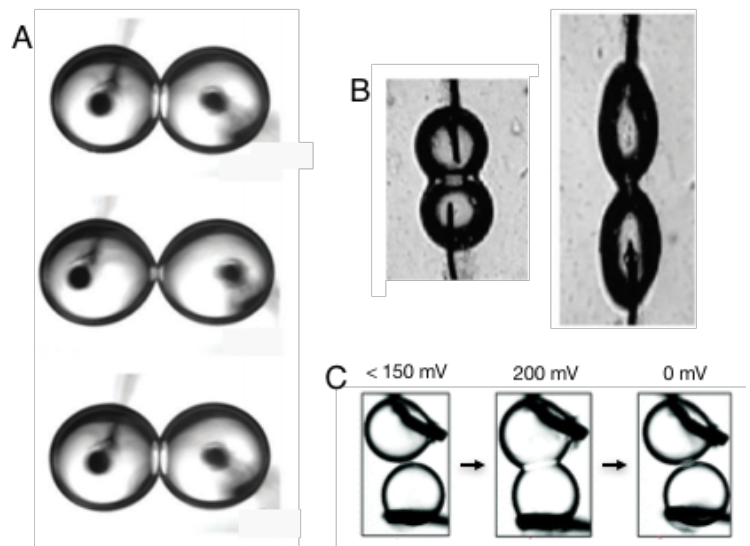

Figure 4. The area of a DIB can be mechanically altered. (A) Once formed, the area of a DIB was altered by pulling on an inserted electrode ${ }^{21}$. (B) A DIB was formed within a deformable PDMS container. When the container was stretched the droplets were also stretched and consequently the bilayer area was reduced ${ }^{34}$. (C) An applied potential produced an increase in the area of a block copolymer bilayer ${ }^{22}$. 
A

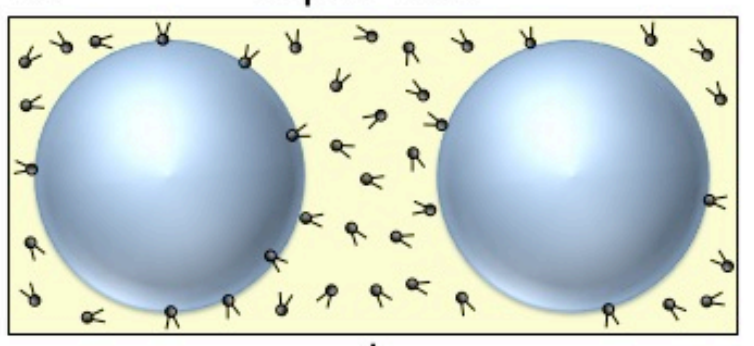

$\downarrow$
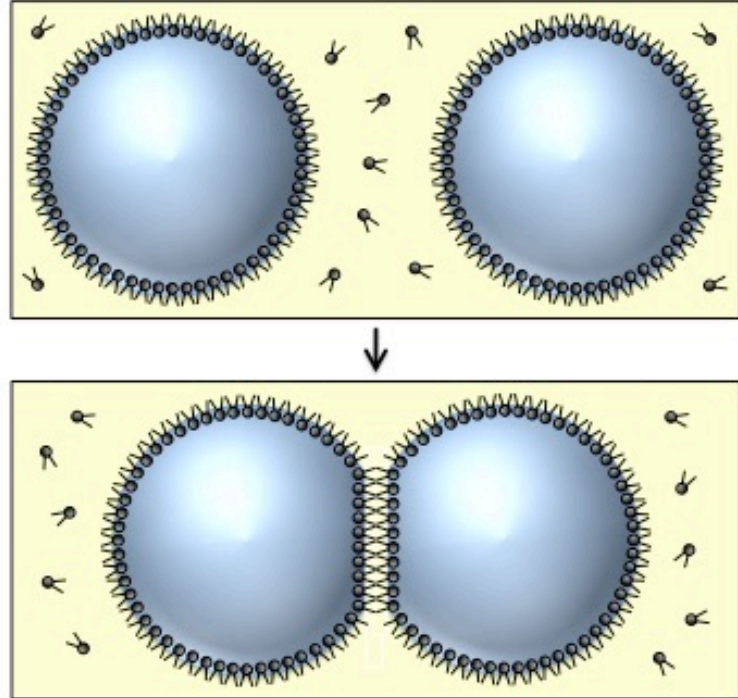

B
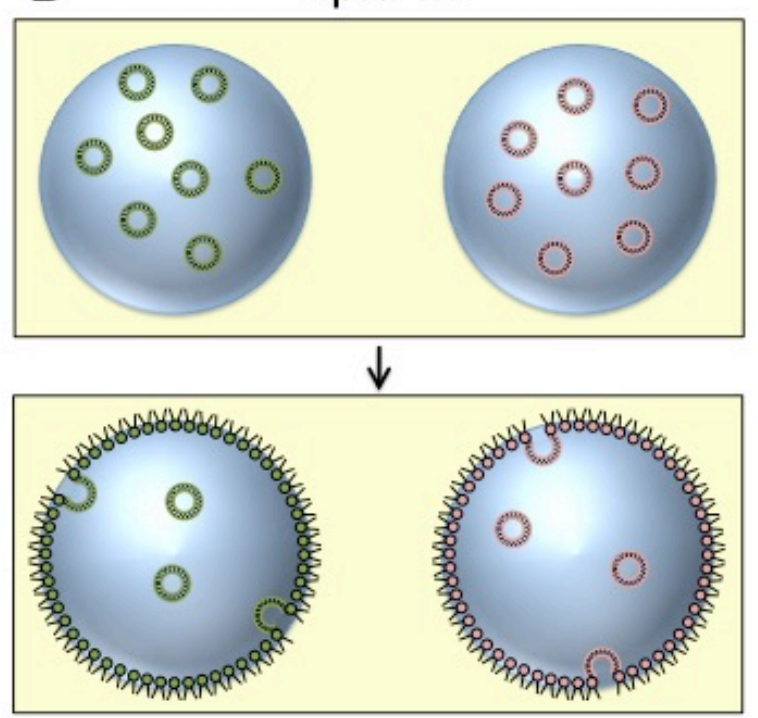

$\downarrow$

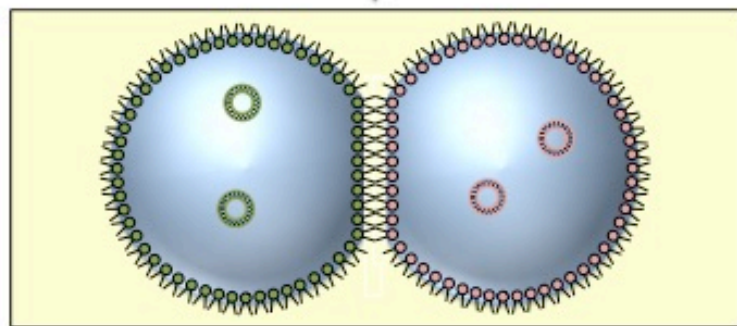

Figure 5. The lipid-out and lipid-in techniques. When DIBs are formed, the lipid can either be (A) dissolved in the oil (lipid-out) or (B) incorporated within the droplets in the form of vesicles (lipid-in). With the lipid-in technique, different lipids (green and pink) can be put in each droplet to create an asymmetric bilayer. 


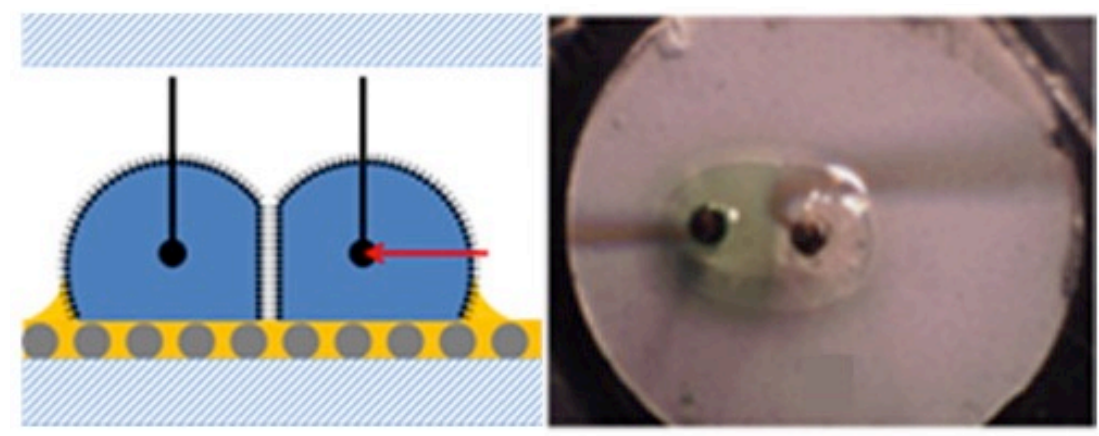

Figure 6. "Air-stable" DIBs. DIBs were formed from aqueous droplets, containing DPhPC liposomes, held on a surface with an oil film (orange) locked into a nanopillared superhydrophobic substrate ${ }^{30}$. The red arrow points to an inserted electrode, which was used to manipulate the DIB and measure its electrical properties. 

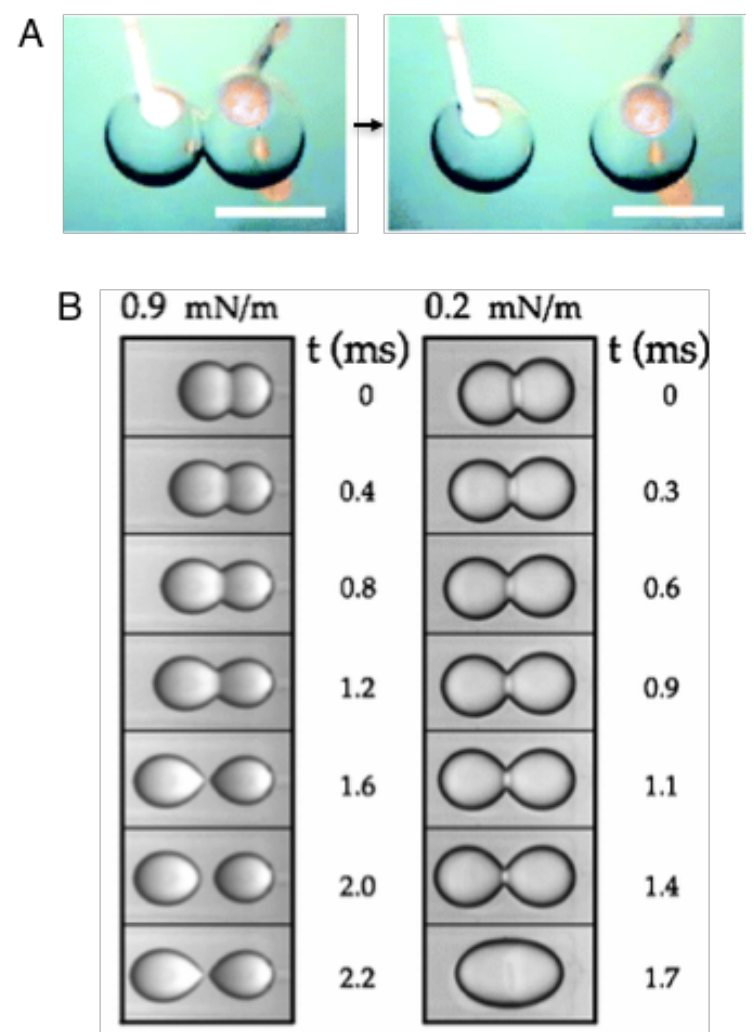

Figure 7. Manipulation of DIBs mechanically and by applying an electric field. (A) Dissociation of a DIB into two separate droplets, by pulling apart electrodes within each droplet ${ }^{3}$. Scale bar, $700 \mu \mathrm{m}$. (B) Two different DIB behaviours under a $5 \mathrm{kV} \mathrm{cm}{ }^{-}$ ${ }^{1} \mathrm{AC}$ electric field. DIBs with a $0.9 \mathrm{mN} \mathrm{m}^{-1}$ bilayer strength unzip and then repel each other, while DIBs of $0.2 \mathrm{mN} \mathrm{m}^{-1}$ bilayer strength coalesce while unzipping ${ }^{19}$. 

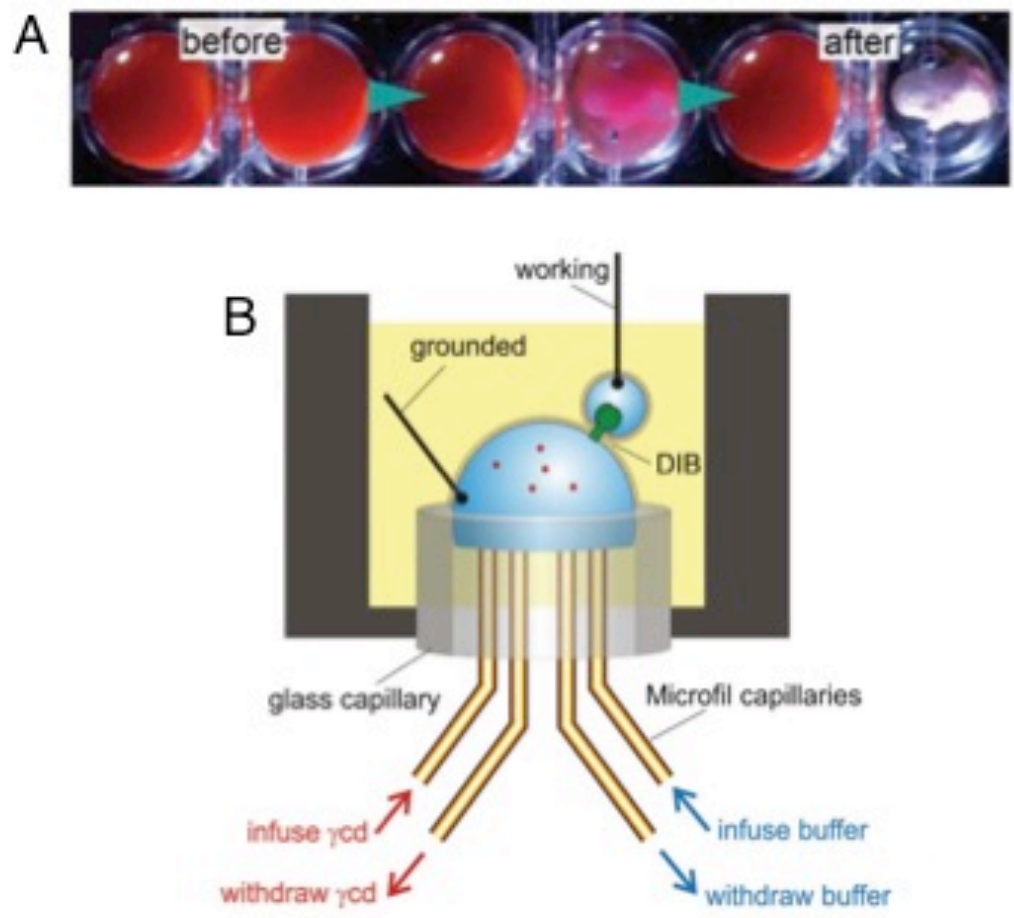

Figure 8. Perfusion of the contents of a single droplet in a droplet pair. (A) A DIB was stabilized by a plastic partition with an aperture between the droplets, which protected the system against mechanical shock and provided a fixed bilayer area ${ }^{50}$. The perfusion of reagents in the droplet on the right-hand side of the DIB was performed with capillaries inserted into the droplet (the green arrow indicates the progression of perfusion). (B) A DIB formed between a mobile droplet on a working electrode and a perfusable droplet set on top of a glass capillary. Four smaller capillaries were connected to four separate syringes to infuse and withdraw solutions from the droplet ${ }^{49}$. 

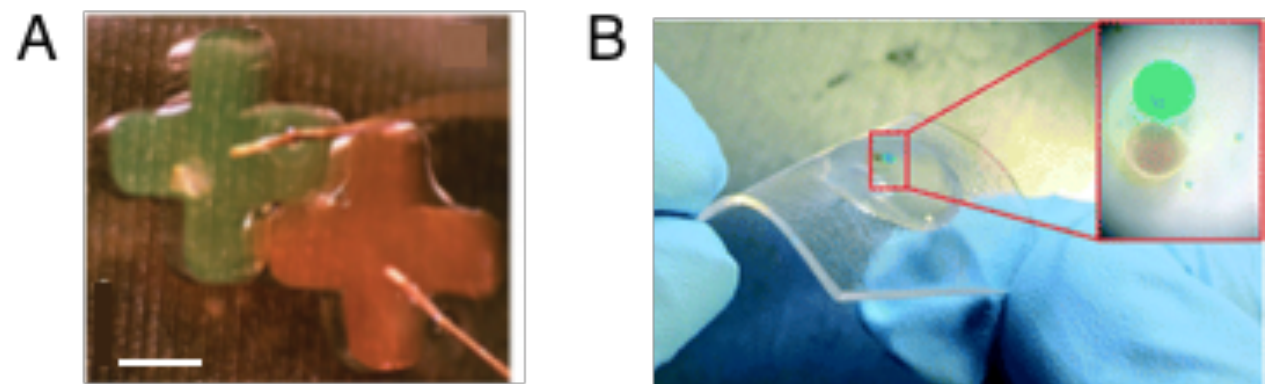

Figure 9. Bilayers with enhanced stability. (A) Two pre-formed hydrogel shapes form a $\mathrm{DHB}^{52}$. Scale bar, $1 \mathrm{~mm}$. (B) DIBs stabilized by using an external organogel composed of hexadecane and a triblock copolymer. The DIB was formed in molten organogel at $50^{\circ} \mathrm{C}$, which was then cooled slowly to room temperature ${ }^{55}$. 
A

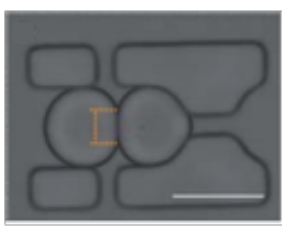

B

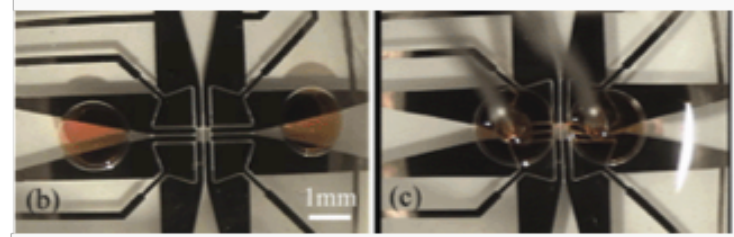

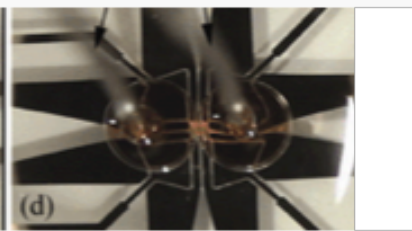

Figure 10. Alternative methods for bilayer formation. (A) DIBs were formed in specially designed traps, from droplets with different contents, at high throughput by using microfluidics ${ }^{59}$. Scale bar, $60 \mu \mathrm{m}$. (B) A DIB was formed and dissociated on planar microelectrodes by using dielectrophoresis ${ }^{25}$. 


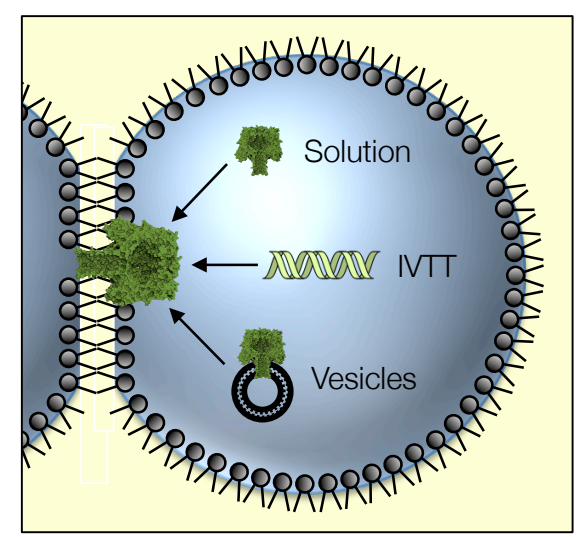

Figure 11. Membrane proteins can be incorporated into DIBs in multiple ways. By using (1) purified proteins, which can spontaneously insert into the membrane when diluted from detergent; (2) in vitro transcription/translation (IVTT), where the protein, encoded by the corresponding DNA, is synthesized inside the droplet; and (3) protein incorporated in vesicle membranes, which fuse with the bilayer. 
A
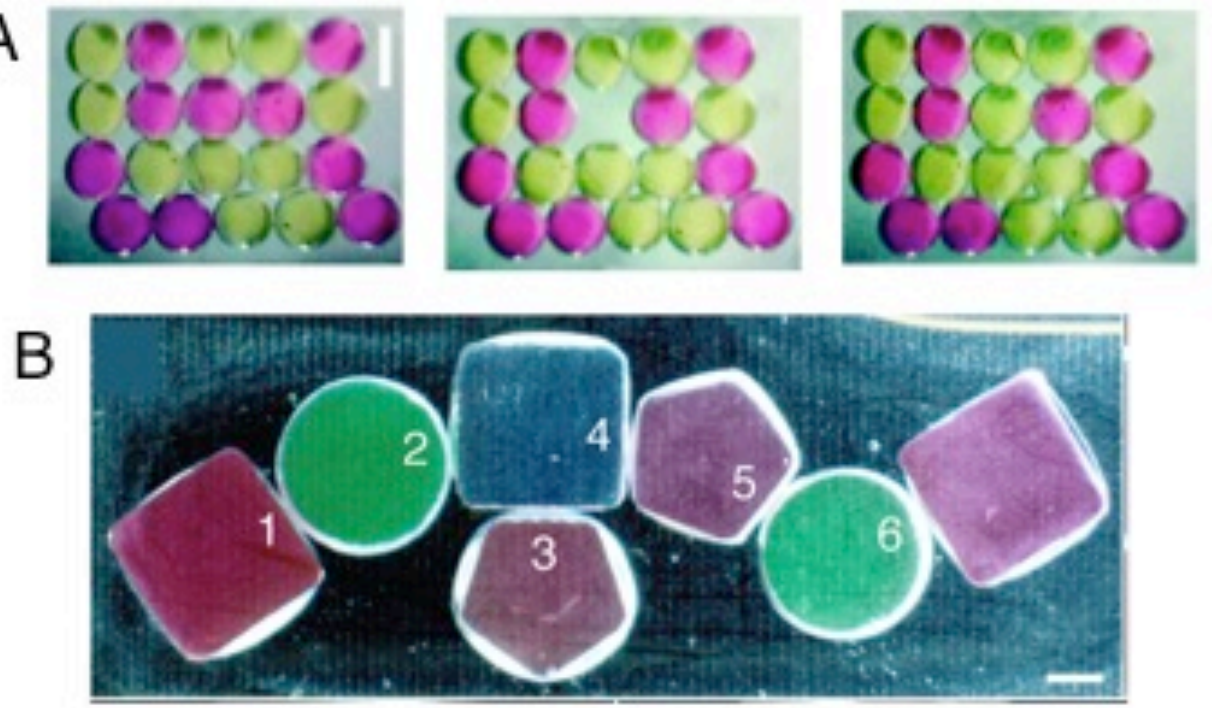

Figure 12. Formation of 2D droplet networks. (A) Droplet placement was facilitated with the use of a micromachined template, resembling an egg box ${ }^{3}$. Here, a single droplet was removed from the network by inserting an agarose-coated electrode and lifting the droplet out of the network. A new droplet was then added in its place. Scale bar, $700 \mu \mathrm{m}$. (B) A small 2D network, containing six bilayers, was made from lipidcoated hydrogel shapes ${ }^{52}$. Scale bar, $1 \mathrm{~mm}$. 

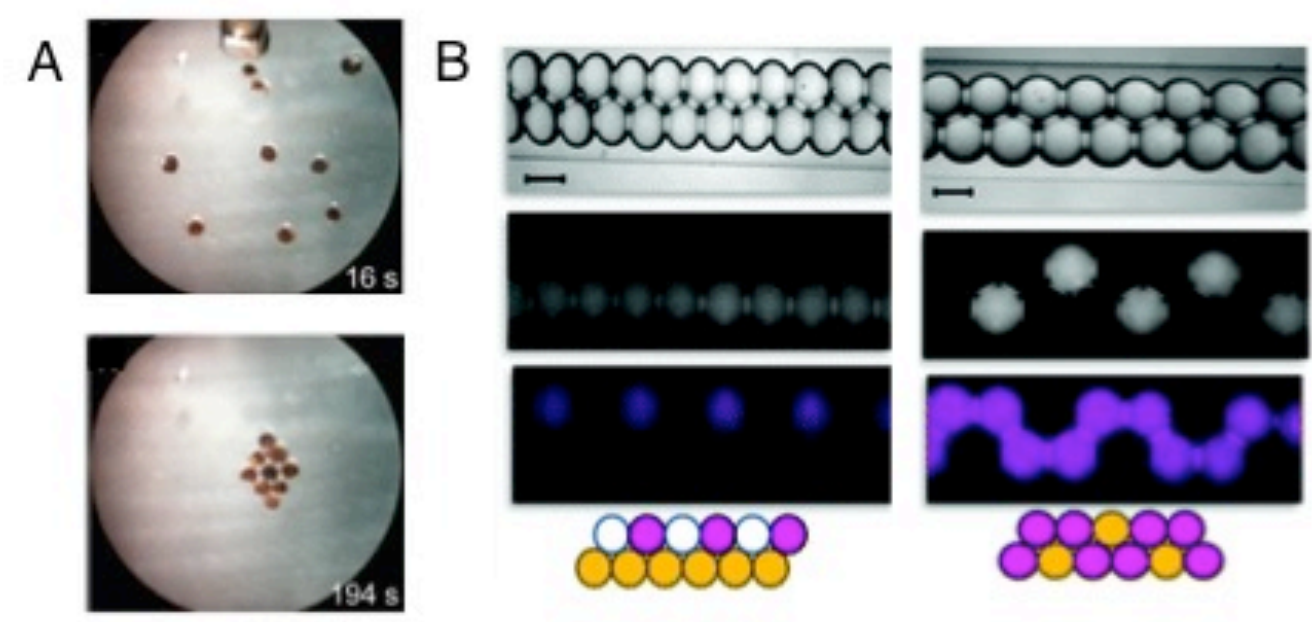

Figure 13. Formation of 2D droplet networks. (A) Droplets containing paramagnetic beads were assembled into a network by using a magnetic probe ${ }^{68}$. (B) Networks of droplets with alternating contents (non-fluorescent or fluorescently-tagged lipid vesicles) were generated with a microfluidic system ${ }^{61}$. Scale bar, $400 \mu \mathrm{m}$. 

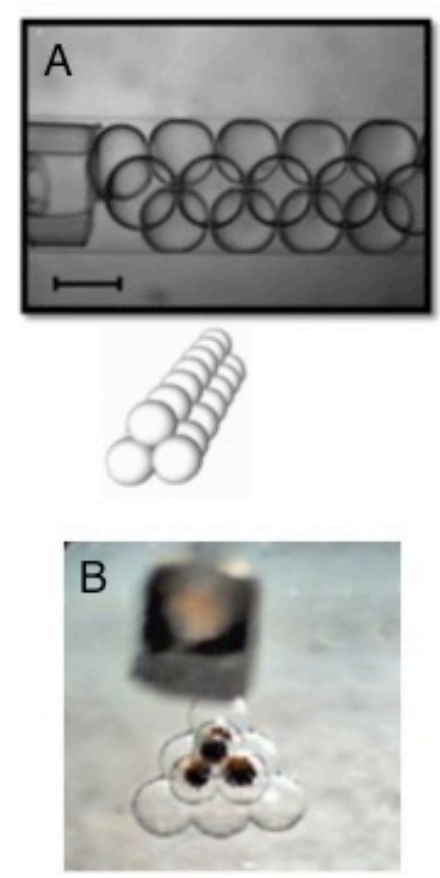
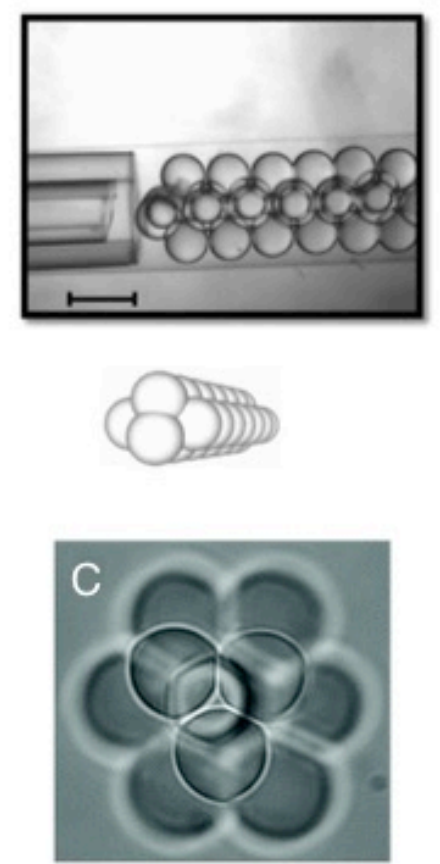

Figure 14. Formation of small 3D droplet networks. (A) 3D droplet networks, in the form of linear chains, were produced by using microfluidics ${ }^{61}$. Scale bar, $400 \mu \mathrm{m}$. (B) A pyramid of up to fourteen paramagnetic droplets was assembled by using a magnetic probe ${ }^{68}$. Scale bar, $200 \mu \mathrm{m}$. (C) A pyramid of eleven identical droplets was brought together by using a single laser-beam optical trap ${ }^{67}$. 

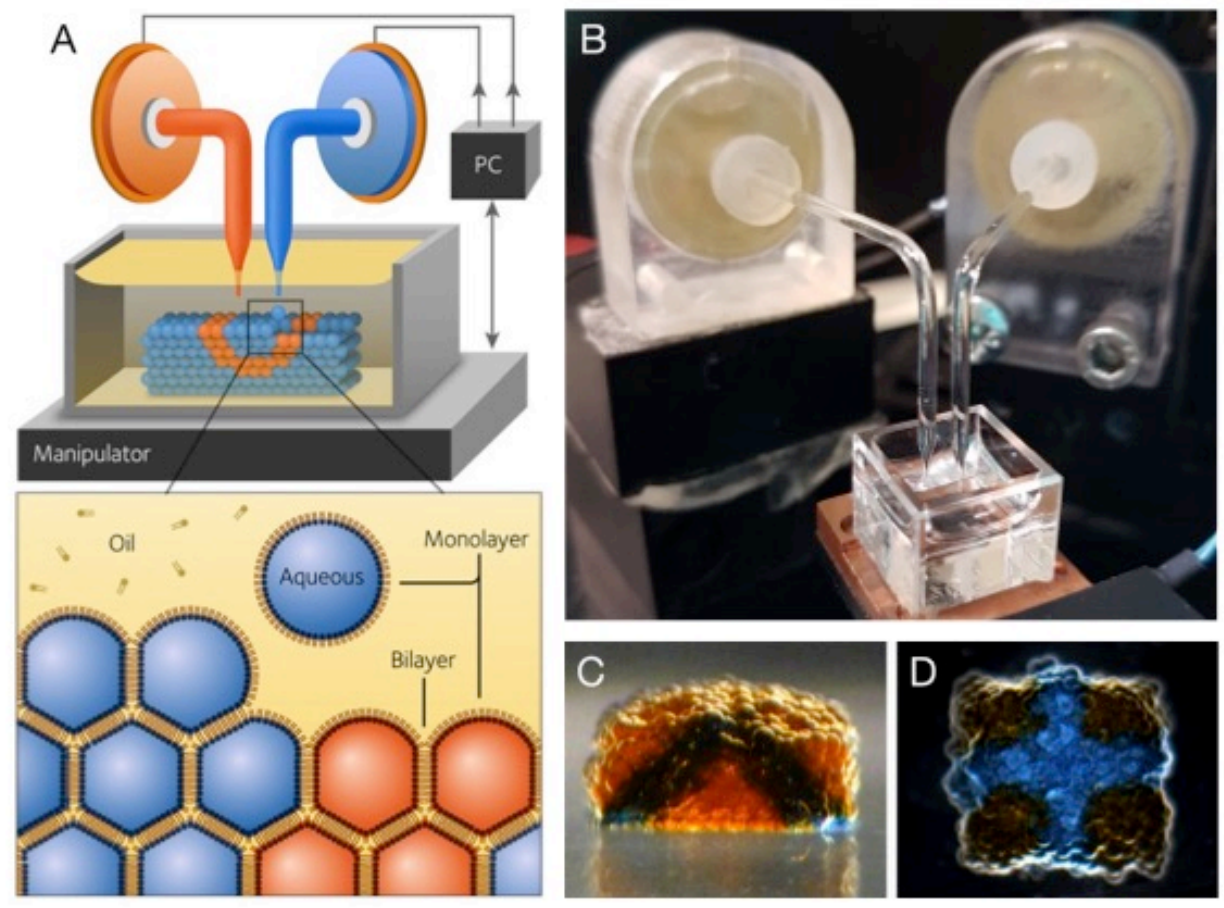

Figure 15. 3D aqueous droplet printer. (A) In this model, two printing nozzles print different droplet solutions into a 3D-patterned droplet network, using piezo transducers (PC) and a micromanipulator, which precisely moves the recipient oil chamber according to a computer program ${ }^{15}$. (B) Image of the 3D droplet printer. (C, D) Two different droplet networks patterned with two droplet types, imaged from the side (C) or the top (D) ${ }^{15}$. 

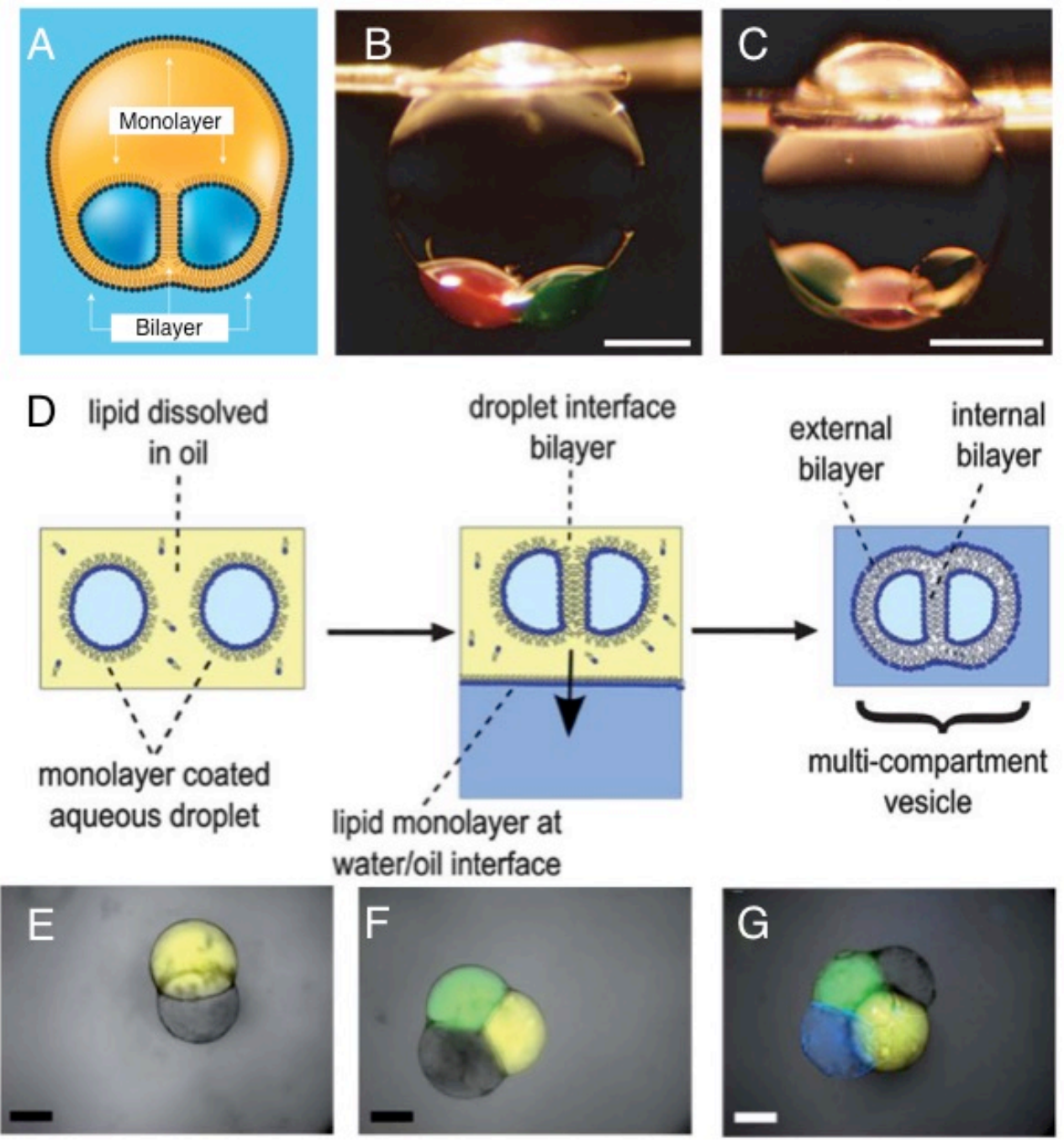

Figure 16. The manual preparation of multisomes. (A) Multisomes were prepared by suspending a lipid-containing oil drop in bulk aqueous solution and then manually pipetting aqueous droplets into it. The interior droplets formed DIBs with one another and bilayers at the external interface ${ }^{89}$. (B) Two-droplet-containing and (C) three-droplet-containing multisomes were prepared with this method $^{89}$. Scale bars, $400 \mu \mathrm{m}$. (D) Pre-formed small droplet networks in a lipid-containing oil were transferred into a bulk aqueous phase by gravity. (E) Two- (F) three- and (G) four-droplet-containing multisomes prepared with this method $^{90}$. Scale bars, $250 \mu \mathrm{m}$. 


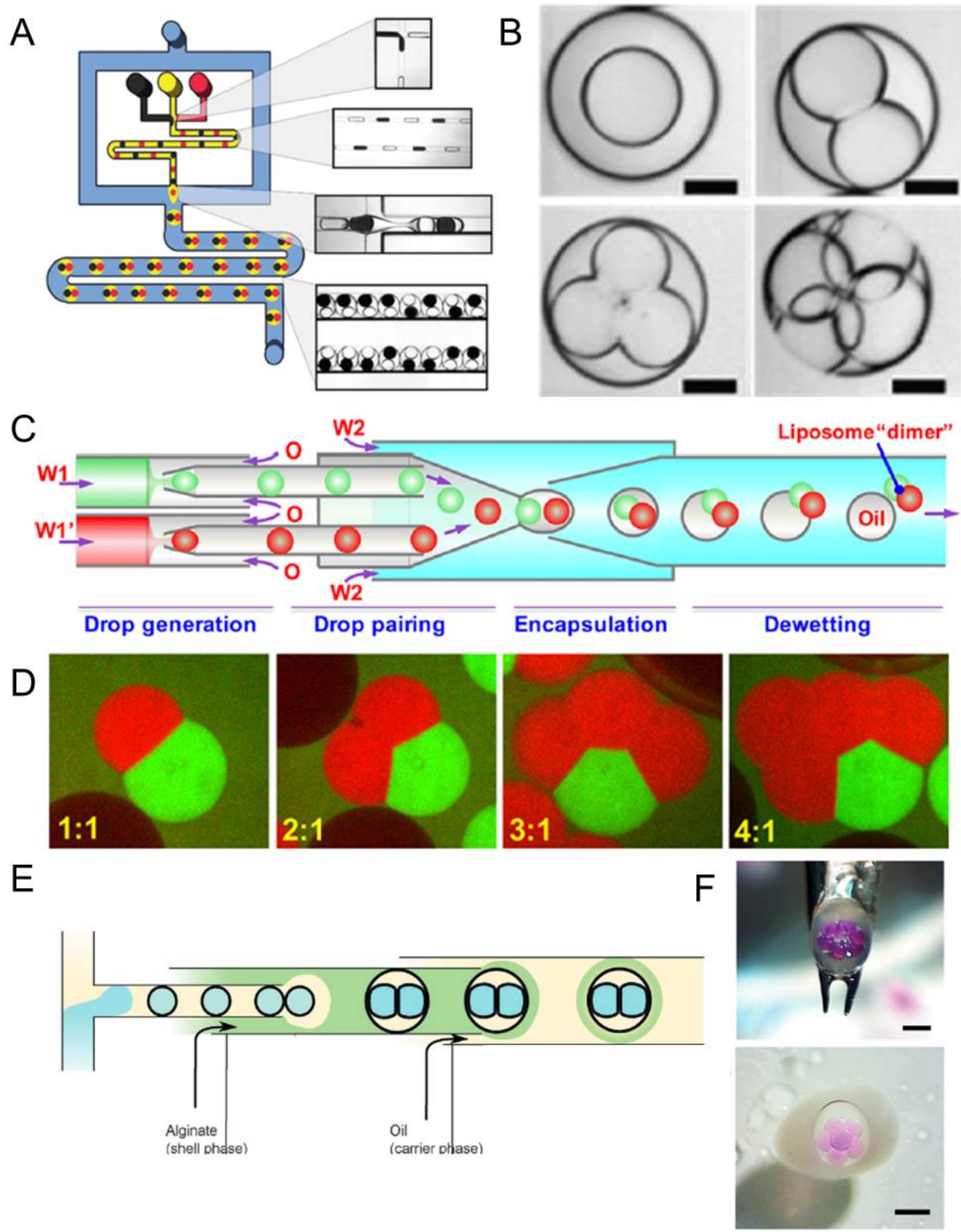

Figure 17. Methods for microfluidic preparation of multisomes. (A) Multisomes containing two different types of droplets were prepared with microfluidics. First, individual aqueous droplets were generated inside a DPhPC-containing oil carrier phase. An oil drop containing aqueous droplets was then ejected into the bulk aqueous phase. (B) Microfluidically prepared multisomes containing 1, 2, 3 and 4 aqueous droplets ${ }^{63}$. Scale bars, $50 \mu \mathrm{m}$. (C) Multisomes with two types of compartments, from which the oil phase spontaneously detaches, prepared with microfluidics. (D) Multisomes with controlled numbers of different internal compartments $^{91}$. (E) Multisomes encapsulated within a hydrogel shell were prepared with microfluidics by a similar process to that shown in (A), with an extra step that generated an alginate shell. $(\mathbf{F})$ These multisomes were robust enough to be transferred into air or a bulk aqueous phase ${ }^{56}$. Scale bars, $1 \mathrm{~mm}$. 

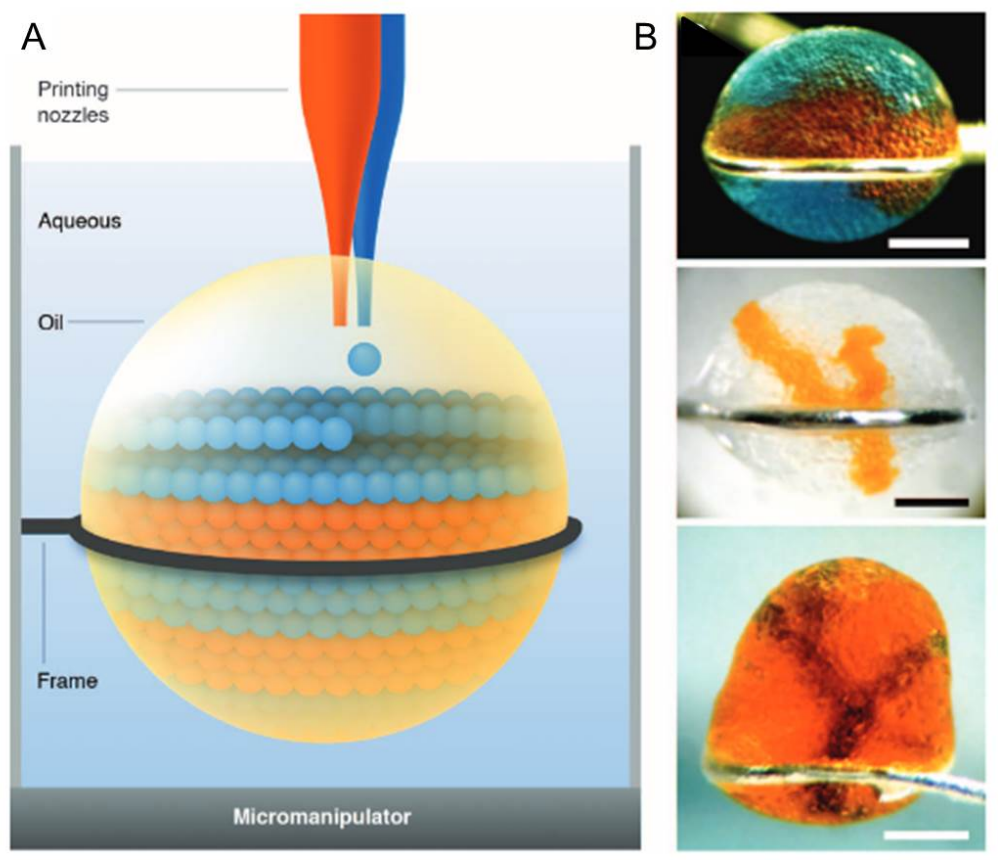

Figure 18. 3D printed multisomes ${ }^{15}$. (A) Printing nozzles were inserted into an oil drop suspended on a frame in bulk aqueous solution. Thousands of $\mathrm{pL}$ droplets were printed within the oil drop. Excess oil was then removed by suction through the printing nozzles. (B) 3D printed multisomes containing thousands of droplets in defined geometries. Scale bars, $400 \mu \mathrm{m}$. 

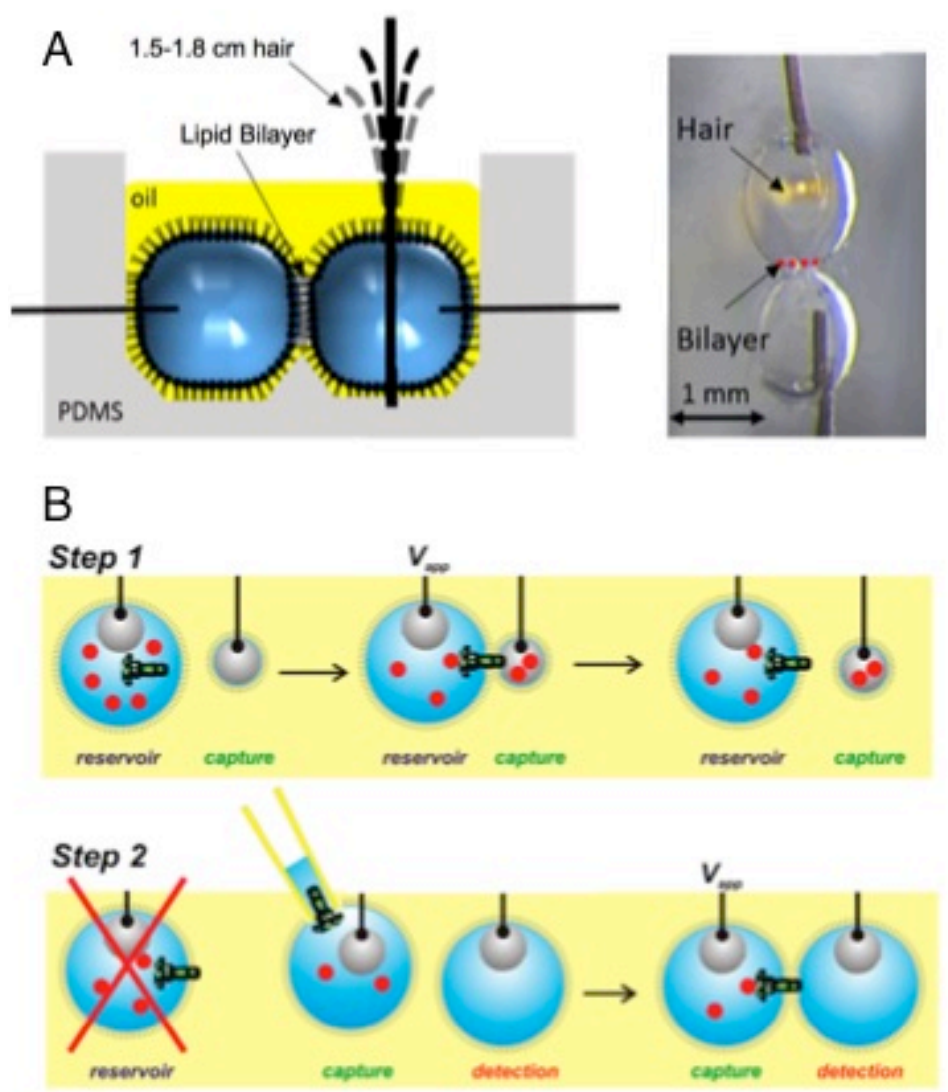

Figure 19. Droplet-based mechanosensors and translocation assays. (A) Artificial "hairs" of different length were placed in one droplet of a DIB. Vibration of the hairs, caused by an airflow, which changed the DIB area, was measured through the associated changes in capacitance ${ }^{93}$. (B) Translocation of the lethal factor of anthrax toxin across a DHB in which the protective antigen (PA) translocator had been incorporated (Step 1). Subsequently, the translocated lethal factor was detected by its ability to block the PA pore in a second DIB assay (Step 2$)^{26}$. 


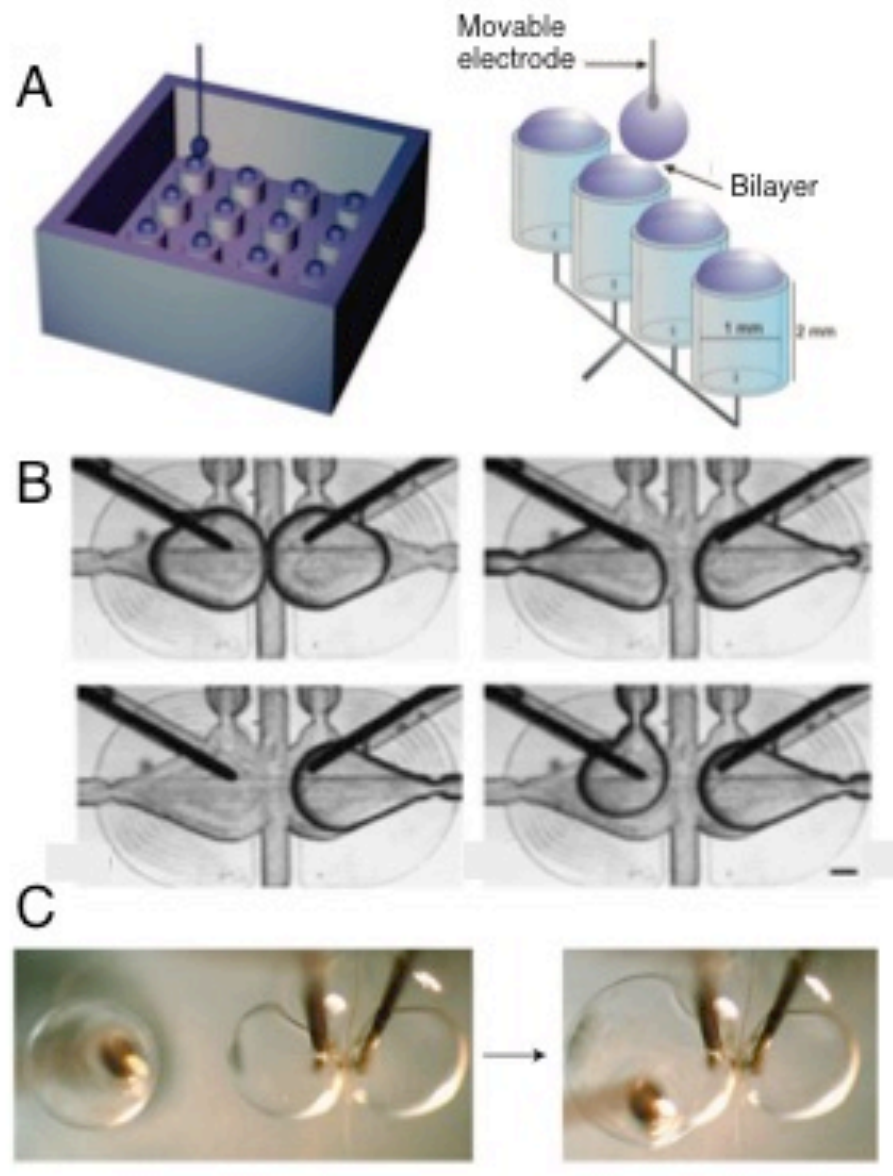

Figure 20. Sequential screening with DIBs. (A) A poly(methyl methacrylate) array containing 16 droplets, loaded with various channel blockers, was scanned manually with an upper a mobile droplet containing a potassium channel. A single bilayer was made at a time ${ }^{24}$. (B) Droplet exchange in a microfluidic device ${ }^{42}$. A DIB was formed in a microfluidic device, with electrodes impaled in both droplets (top left). Using microfluidics, the left droplet was removed from the electrode (top right) to leave an empty electrode (bottom left), while the right droplet was trapped on an electrode. A new droplet was then impaled on the empty electrode, to form a new DIB (bottom right). Scale bar, $200 \mu \mathrm{m}$. (C) A droplet-fusion system allowed the introduction of new material into one droplet of a DIB ${ }^{48}$. This permitted the screening of the same membrane protein under several conditions. 

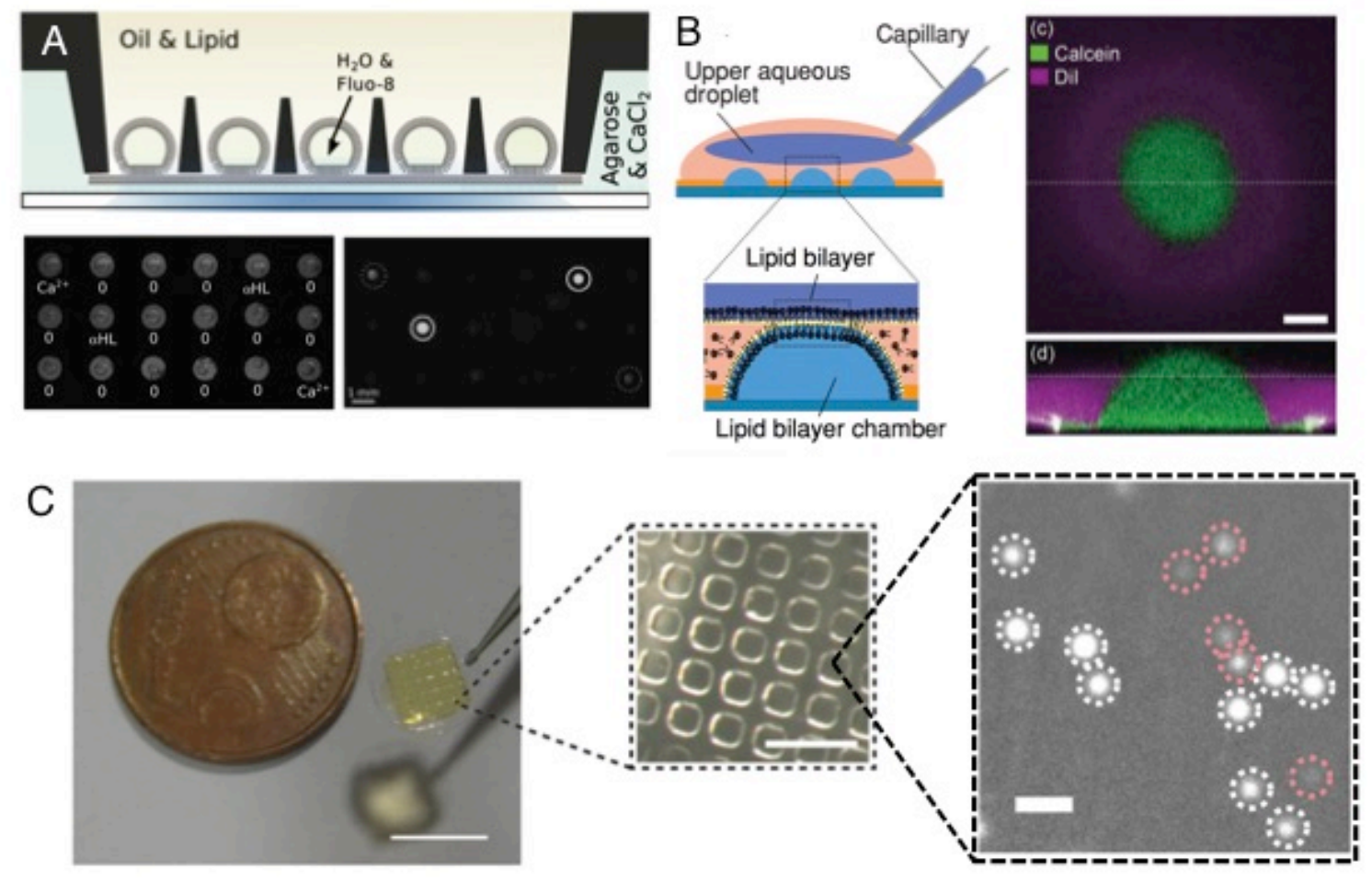

Figure 21. Parallel observation of pore activity in droplet and hydrogel interface bilayers. (A) Fifteen DHBs were formed by placing a PMMA array (black) on top of a hydrogel surface (blue) and adding droplets to each well of the array. $\mathrm{Ca}^{2+}$ diffusion through ensembles of $\alpha \mathrm{HL}$ pores was measured in each recipient droplet with the dye fluo- $8^{116}$. Scale bar, $1 \mathrm{~mm}$. (B) An aqueous droplet coated with a lipid monolayer was placed on top of an array of 36 lipid-coated aqueous droplets. This allowed the fluorescence detection of $\mathrm{Ca}^{2+}$ flux through $\alpha \mathrm{HL}$ pores in all the DIBs simultaneously ${ }^{117}$. Scale bar, $10 \mu \mathrm{m}$. (C) An array of thousands of hydrogel pillars was brought into contact with a hydrogel surface. $\mathrm{Ca}^{2+}$ flux under a transmembrane potential through hundreds of individual $\alpha \mathrm{HL}$ pores was monitored by total internal reflection (TIRF) microscopy ${ }^{120}$. Scale bars, $8 \mathrm{~mm}, 140 \mu \mathrm{m}$ and $10 \mu \mathrm{m}$, respectively. 
A
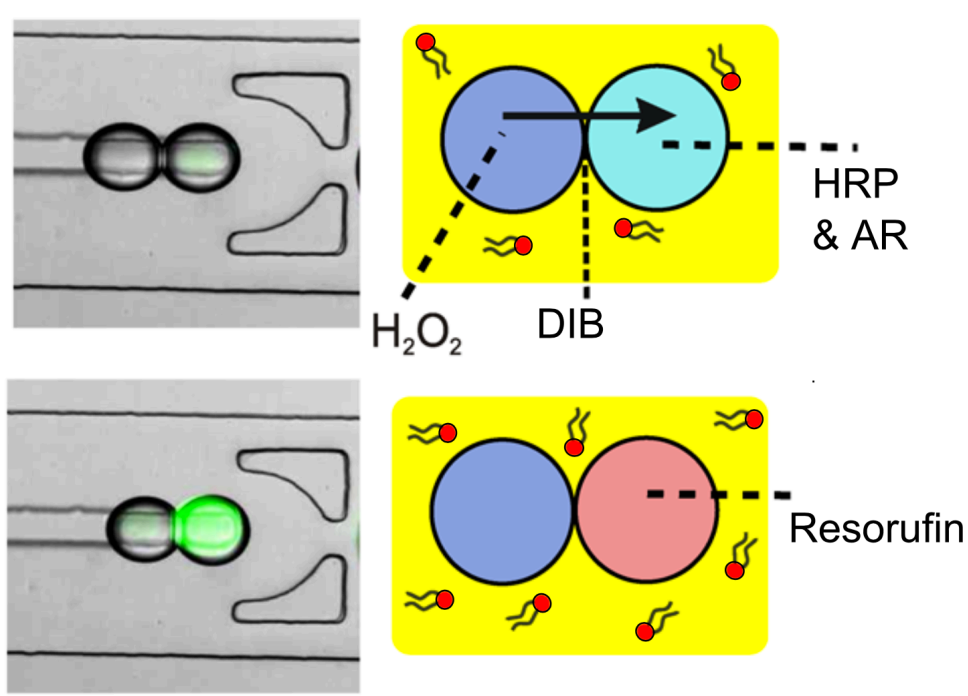

B

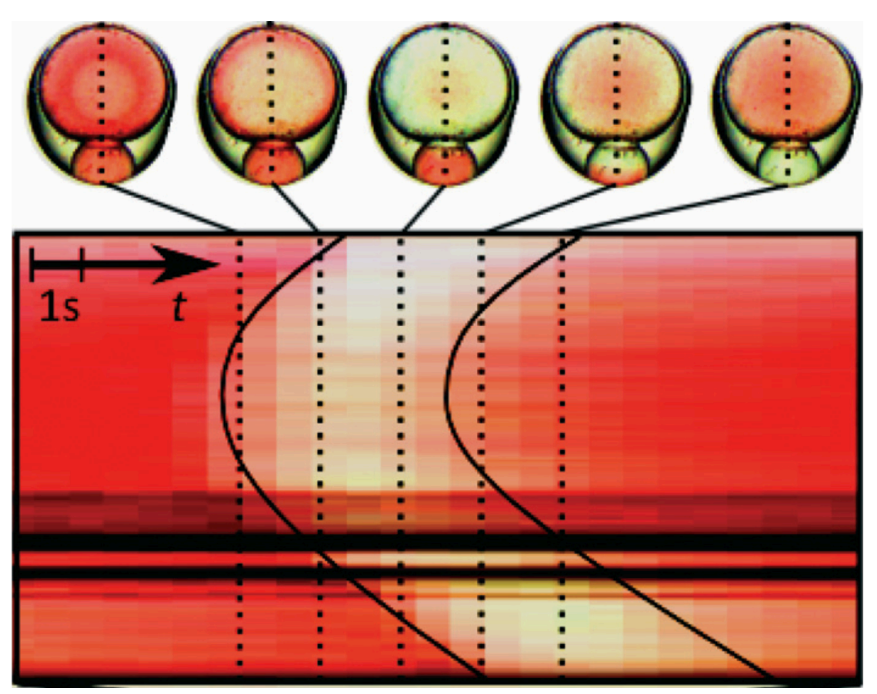

Figure 22. Chemical reactions in DIBs. (A) A bilayer was formed between one droplet containing hydrogen peroxide and a second containing the enzyme horseradish peroxidase (HRP) and amplex red (AR). Upon DIB formation, the hydrogen peroxide diffused across the bilayer and acted as a substrate for the horseradish peroxidase to catalyse the formation of the fluorescent product resorufin from $\mathrm{AR}^{85}(\mathrm{~B})$ The $\mathrm{BZ}$ chemical reaction can travel across a $\mathrm{DIB}^{127}$. Line profiles across the DIB were combined in a time-plot. The chemical wave of the reaction expanded from the center of the larger droplet. The wave then propagated outwards, passing through the bilayer and into the smaller droplet. 

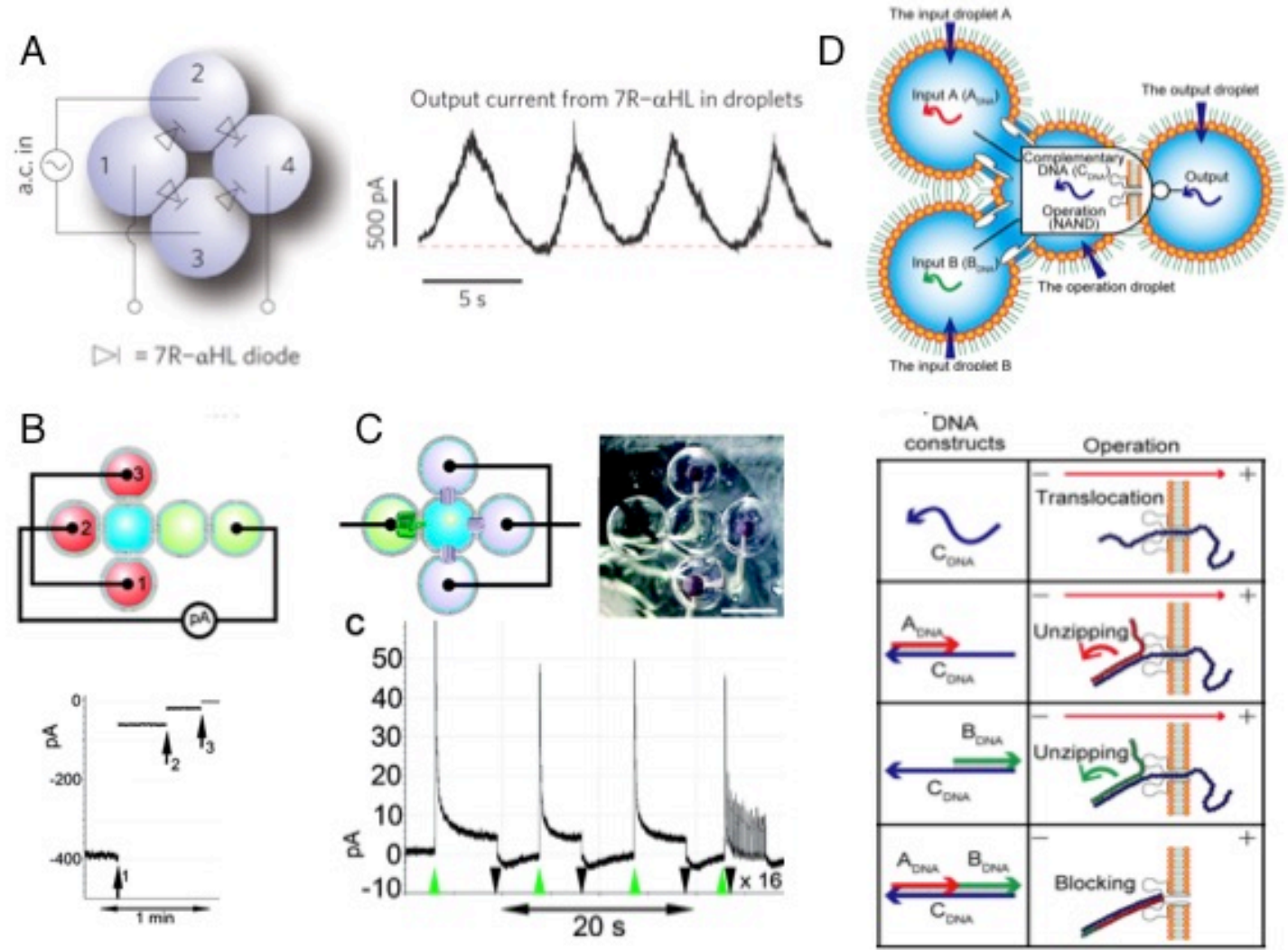

Figure 23. Electronic devices made from 2D droplet networks. (A) A fourdroplet network that functions as a full-wave rectifier was created by using a mutant $\alpha \mathrm{HL}$ pore that acts as a diode ${ }^{142}$. (B) A biobattery based on a concentration cell was constructed from three input droplets (red) in a 6droplet network ${ }^{3}$. A transmembrane potential was generated in the network by incorporating an engineered anion-selective $\mathrm{aHL}$ pore into the red input droplets, which allows a higher flux of $\mathrm{Cl}^{-}$ions relative to $\mathrm{Na}^{+}$ions. To complete the circuit the blue droplet contained no pores and the green droplets contained wild-type $\alpha \mathrm{HL}$ pores. The red droplets contained a higher concentration of $\mathrm{Cl}^{-}$than the blue droplet. As each of the three input droplets was removed from the network (steps 1,2 and 3) the current was reduced in steps to $0 \mathrm{pA}$. (C) The light-driven proton pump bacteriorhodopsin (bR), from Halobacterium salinarum, was used to generate power in a five-droplet network ${ }^{3}$. Three bR-containing droplets (purple) were connected to a common droplet containing no protein (blue), which was in turn connected to a $\alpha \mathrm{HL}-$ containing droplet (green) to complete the circuit. When green light illuminated the network (green arrows) proton pumping across the DIBs between the purple and blue droplets was measured as an increase in current. Scale bar $700 \mu \mathrm{m}$. (D) A NAND (negative-AND) gate was developed from a four-droplet network. Three droplets, two input and one output, were each connected to a common central operation droplet ${ }^{147}$. The two input droplets contained singlestranded DNA, complementary to a DNA strand in the central droplet. When both inputs were present, a double-stranded DNA was formed, which blocked a pore in the DIB between the operation and output droplets. If no strands or only one of the strands was present, the partial DNA duplex formed could translocate through the pore, which therefore was not blocked. 

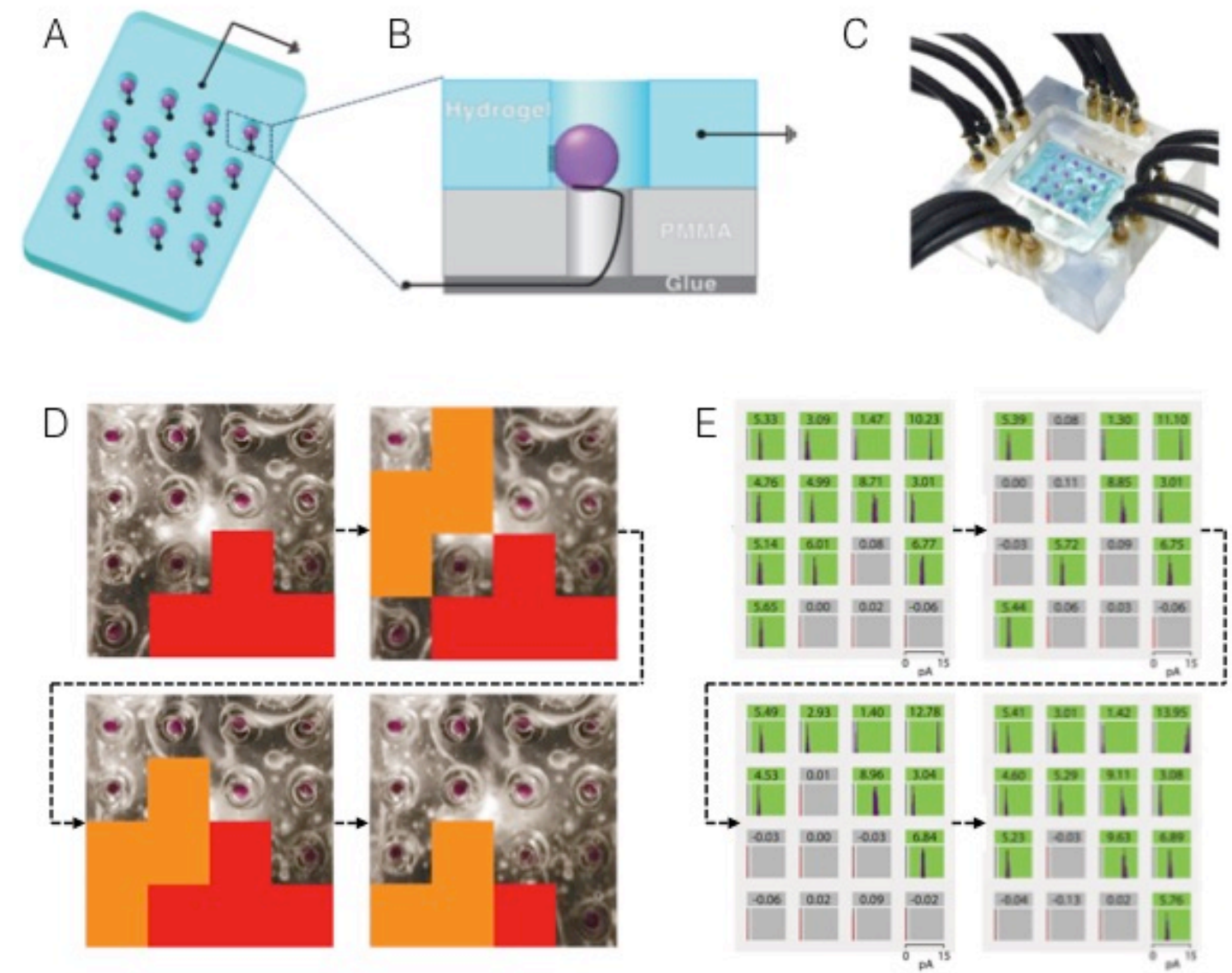

Figure 24. Patterned current generation within a droplet bilayer array ${ }^{144}$. (A) Schematic representation of a $4 \times 4$ droplet array, containing sixteen bRcontaining droplets patterned within a hydrogel structure. Each droplet was connected to a separate electrode, while the hydrogel was connected to a single common ground electrode. (B) A cross-section of a single droplet within the array shows the bilayer between the droplet and the hydrogel. (C) Image of the 4x4 droplet array, showing the PMMA chamber used to hold the droplet array and the 16 cables connected to electrodes in each droplet. (D) An image of the bilayer array from above, with a schematic of two Tetris-based shapes moving down the face of the droplet array. (E) By measuring the current output of each of the droplets (coloured green when $>1 \mathrm{pA}$ and grey when $<1 \mathrm{pA}$ ), the shapes could be detected as they blocked the light from reaching the droplets below them. 

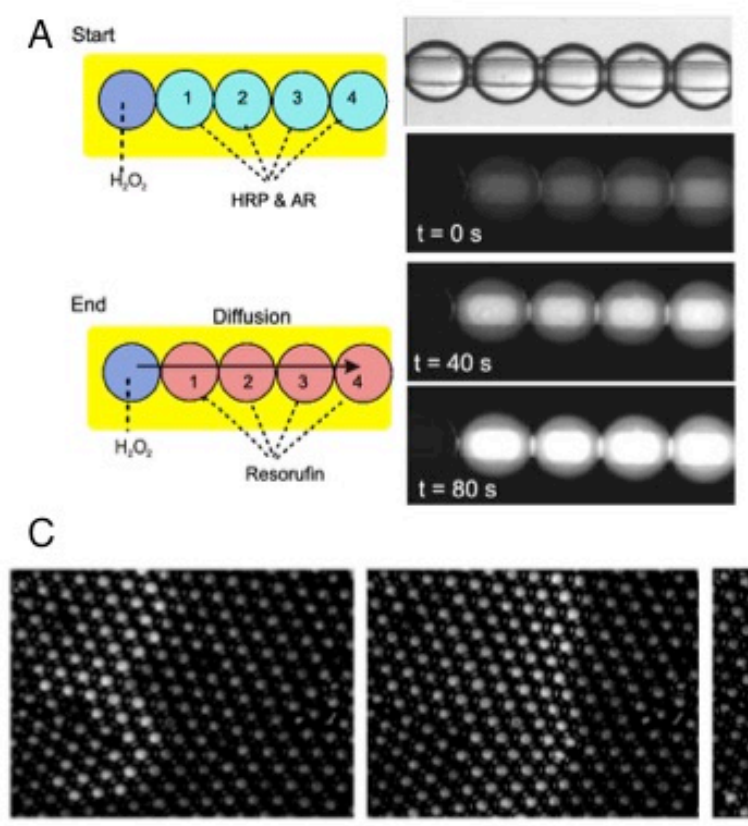
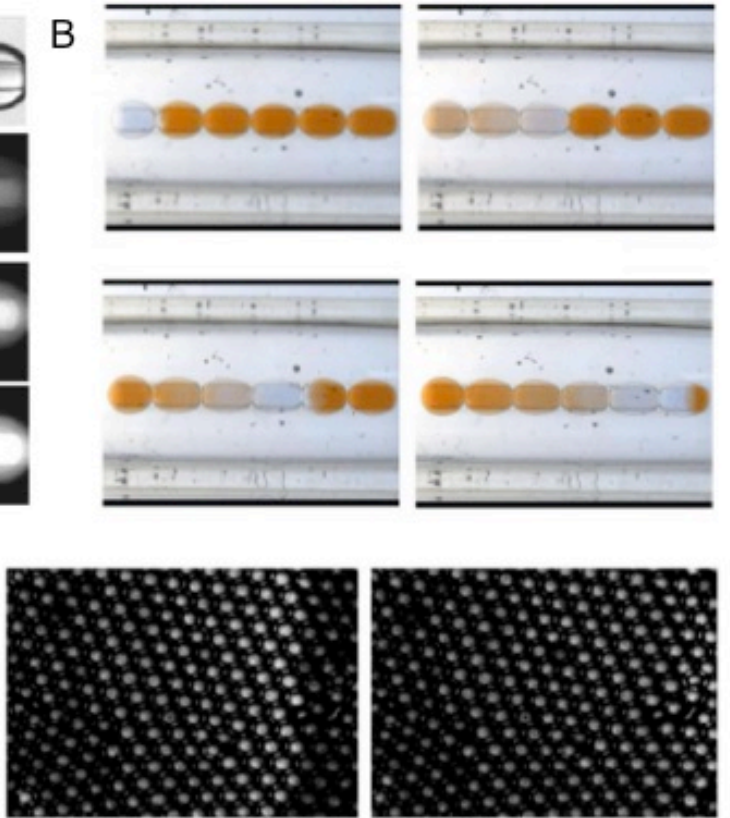

Figure 25. Chemical reactions in droplet compartments. (A) Hydrogen peroxide, contained in a single droplet, diffused across a linear network of DIBs. The recipient droplets contained Amplex Red and the enzyme horseradish peroxidase, which catalysed the formation of the fluorescent product resorufin ${ }^{62}$. Droplets were $100 \mu \mathrm{m}$ in diameter. (B) The BZ chemical reaction travelled across six DIBs in a linear chain of droplets ${ }^{84}$. Droplets were held in a $1 \mathrm{~mm}$ wide trench. (C) Travelling waves of the BZ reaction observed through large 2D sheets of bilayer-connected droplets ${ }^{126}$. Droplets were 30 $\mu \mathrm{m}$ in diameter. 

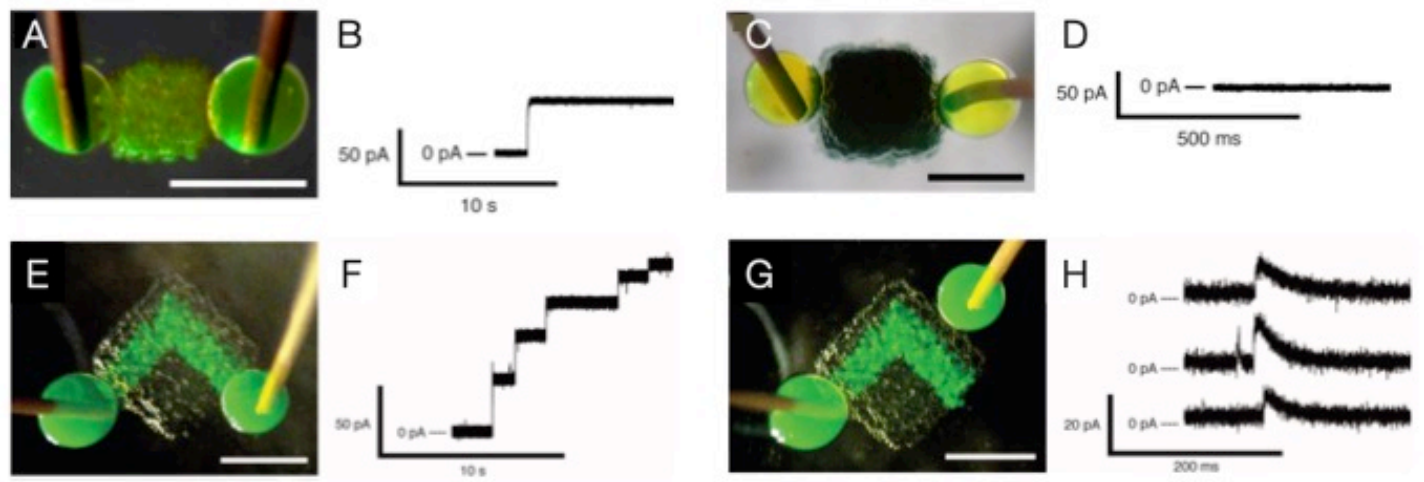

Figure 26. Electrical communication through 3D printed networks ${ }^{15}$. (A-D) lonically conductive 3D-printed droplet networks. (A, B) Electrode-containing droplets formed bilayers with either side of a network in which all the droplets contained the aHL pore. When a voltage was applied, an increasing electrical current signal was observed as $\mathrm{aHL}$ pores inserted into each of the electrode droplets. (C, D) When the same experiment was performed with a network that did not contain aHL, no current signal was observed. (E-H) Droplet network printed with an ionically conductive pathway (green droplets). (E, F) When electrode-containing droplets formed bilayers with either end of the pathway and a voltage was applied, an increasing electrical current signal was observed. (G, H) When one of the electrode-containing droplets was placed on the network away from the pathway and a voltage applied, only weak transient current signals were observed. Scale bars, $500 \mu \mathrm{m}$. 
A
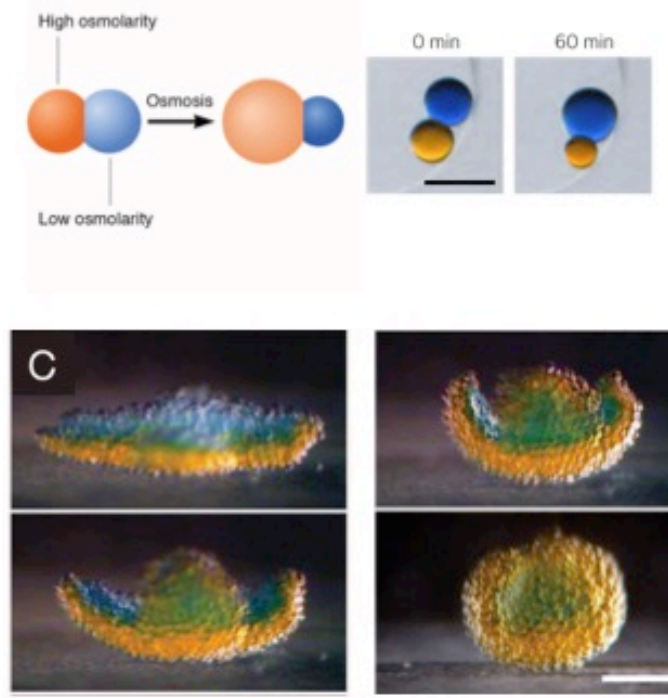

$\mathrm{B}$
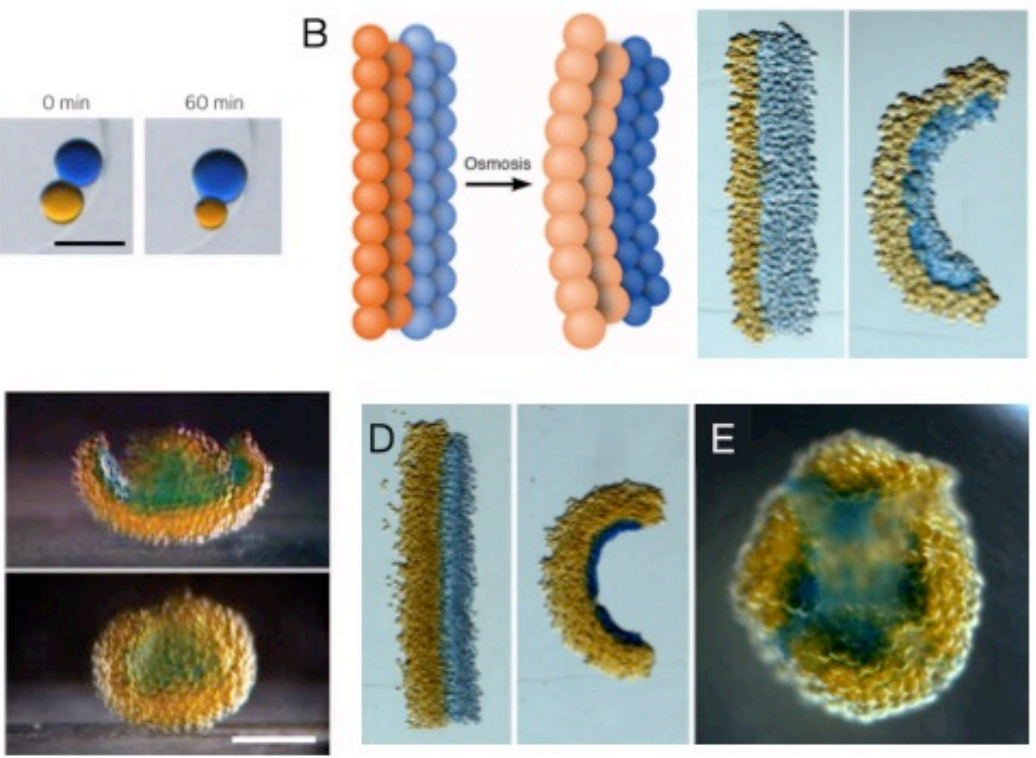

Figure 27. Folding 3D-printed networks ${ }^{15}$. (A) Two droplets of different osmolarities change their volumes over time as water moves across the bilayer (Scale bar, $1 \mathrm{~mm}$ ). (B) Water flow in networks containing two strips of droplets with different osmolarities causes the network to fold. (C) A flat petalshaped network, printed with layers of droplets with different osmolarities (orange $=$ high, blue $=$ low), folded into a hollow sphere because of water movement across the bilayers (Video of this process is available online ${ }^{15}$ ).

Scale bar, $200 \mu \mathrm{m}$. (D) A network composed of two strips of droplets similar to that in $\mathbf{B}$, except that the width of the strip of droplets of lower osmolarity in this network was thinner. After completion of folding, the network has fractured in the region of the blue droplets. (E) Folding of a petal-shaped network similar to that in $\mathbf{C}$. The layer of droplets with a lower osmolarity fractured near the base of the upper arm, causing the arm to buckle and fold to a lesser extent than the others. 

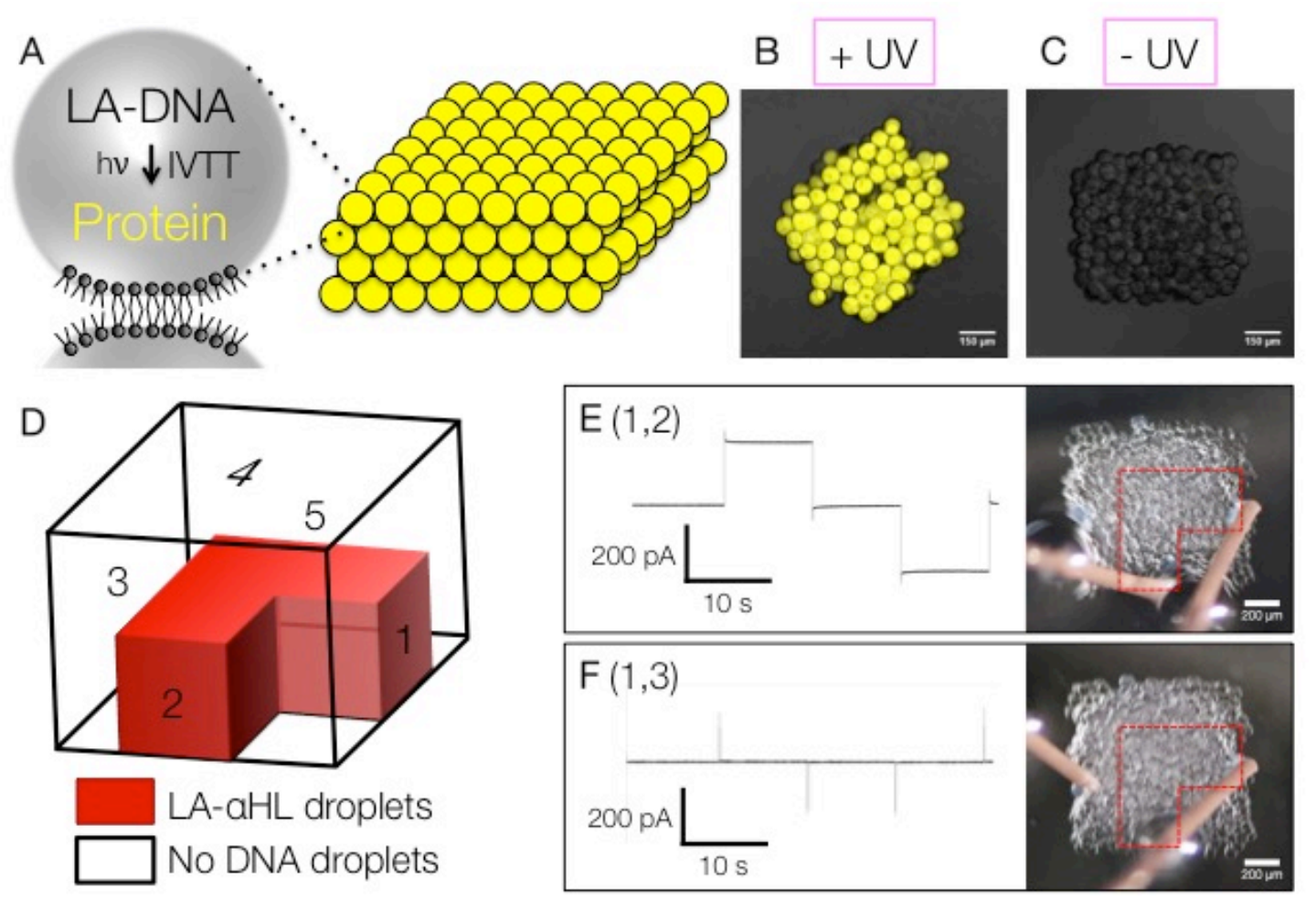

Figure 28. Controlling 3D-printed synthetic tissues with light ${ }^{16}$. (A) Droplets containing LA-DNA and an in vitro transcription/translation (IVTT) system (synthetic cells), can be printed into a 3D droplet network (synthetic tissue). Protein expression only occurs in the synthetic tissue following UV activation of the DNA. (B) Fluorescent protein expression was observed in networks following UV treatment. (C) No expression was observed without UV treatment. (D) Synthetic tissue printed with a 3D pathway (red) of synthetic cells containing LA-aHL DNA. UV activation caused the expression of $\mathrm{aHL}$, forming a 3D electrically conductive pathway. Electrical communication was observed when two electrodes were held at either end of the pathway $(\mathbf{E})$. No current was detected when one electrode was placed on the surface of the network away from the pathway $(\mathbf{F})$. 
A

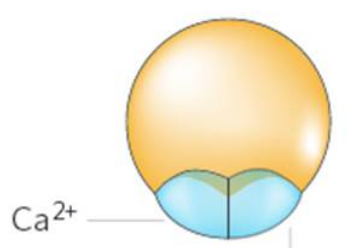

Fluo-4

Fluorescent mixture
B
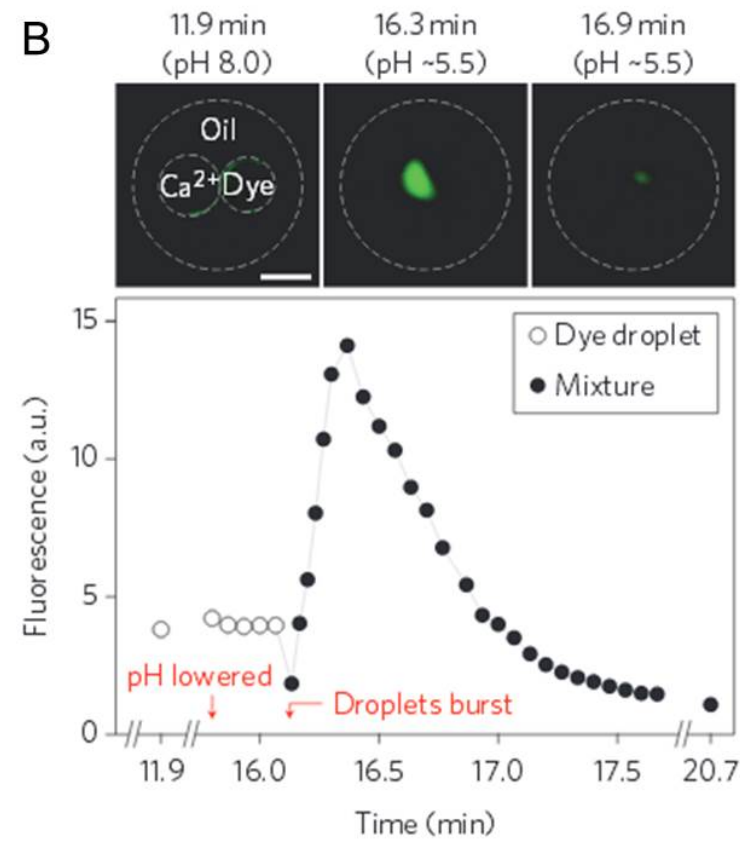

Figure 29. Communication between the internal compartments of multisomes and the external environment ${ }^{89}$. (A) Controlled mixing of a two-compartment multisome with one droplet containing $\mathrm{Ca}^{2+}$ and the other Fluo-4, a fluorogenic $\mathrm{Ca}^{2+}$-binding dye. By using functionalised lipids, a change in temperature or $\mathrm{pH}$ triggers the rupture of the internal and external bilayers, causing the internal contents of the multisomes to be expelled and mix in the external aqueous environment. (B) When $\mathrm{pH}$-sensitive bilayers were used, a drop in $\mathrm{pH}$ caused a transient increase in fluorescence, as the Fluo-4 bound to the $\mathrm{Ca}^{2+}$ before diffusion into the bulk aqueous phase. Scale bar, $500 \mu \mathrm{m}$. 
A

A

1

2

Lactase

glucose

B
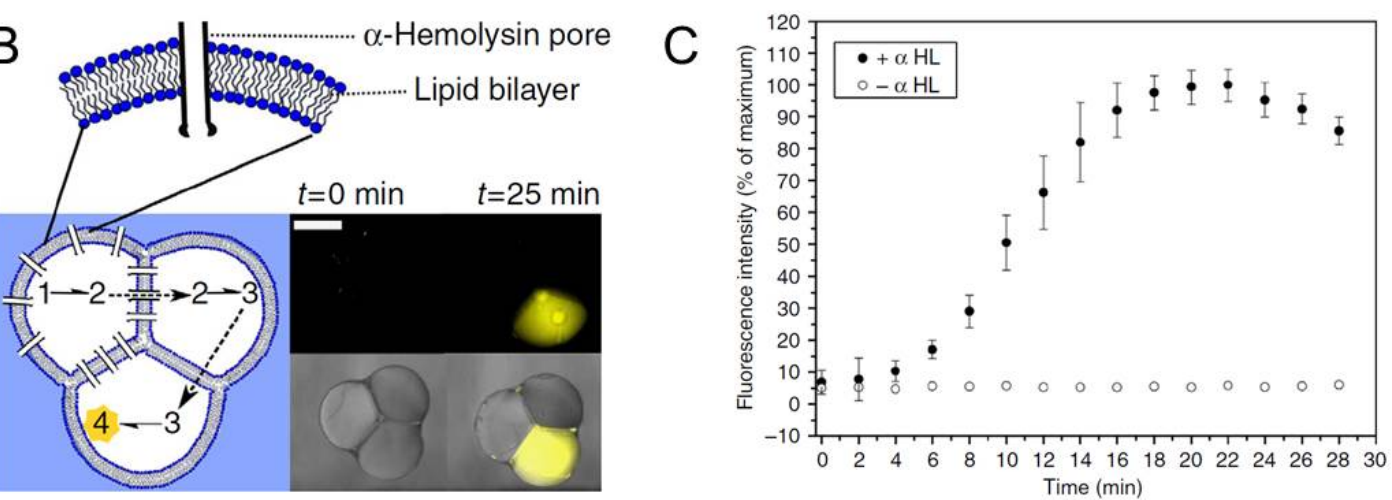

Figure 30. Multisome-encapsulated enzymatic pathway ${ }^{125}$. (A) Reaction scheme for the enzymatic pathway. (B) Lactose (1) is hydrolysed to D-glucose (2), which diffuses through aHL pores into a second compartment and is oxidised to form hydrogen peroxide (3). Hydrogen peroxide then diffuses into a third compartment, where it reacts with Amplex Red to form the fluorescent resorufin (4). (C) Normalized fluorescence measurements of multisomes, with and without aHL. Scale bars, $250 \mu \mathrm{m}$. 
A
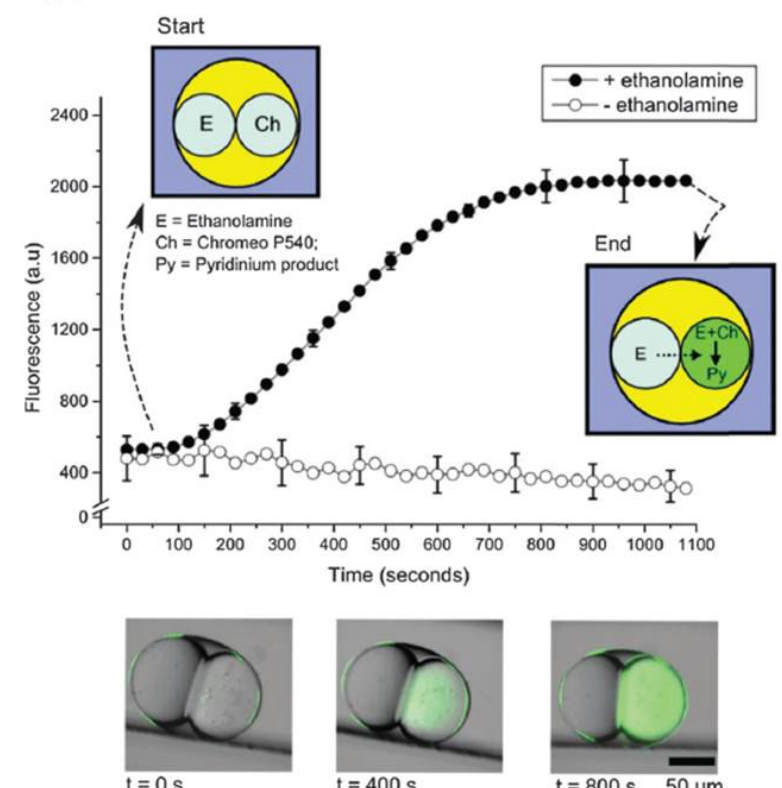

B

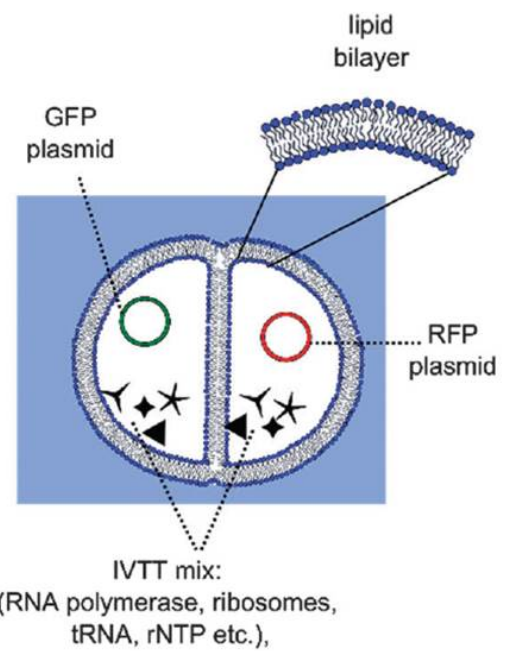

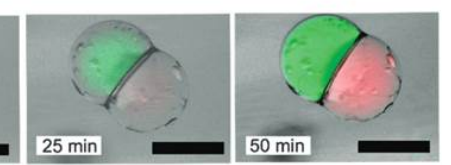

Figure 31. Multisome-encapsulated reactions. (A) A multisome containing two droplets was made, one contained ethanolamine and the other Chromeo P540. Ethanolamine diffused across the bilayer and reacted with the Chromeo P540 to form a fluorescent pyridinium product ${ }^{63}$. Scale bar, $50 \mu \mathrm{m}$. (B) A 2-compartment multisome was prepared that contained a cell-free expression system and a different plasmid in each compartment. A different fluorescent protein was synthesised inside each compartment ${ }^{74}$. Scale bar, $200 \mu \mathrm{m}$. 

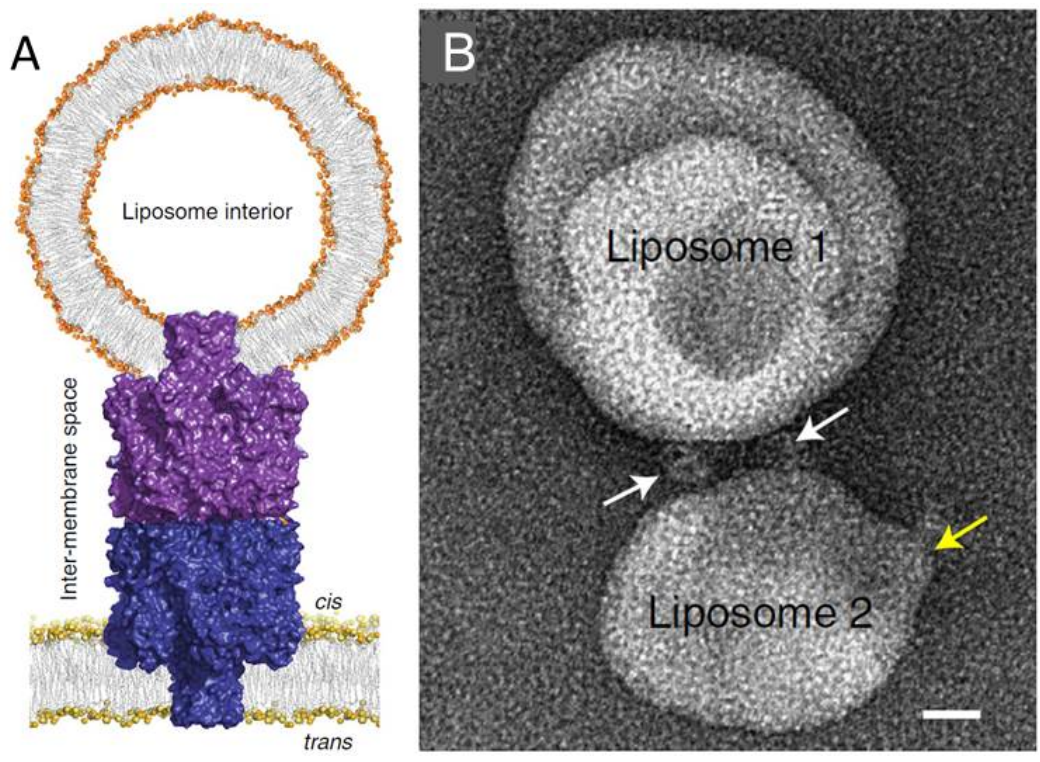

Figure 32. The bridging of two bilayers by the hemolysin dimer ${ }^{167}(a 7)_{2}$. (A) Cartoon showing the simultaneous insertion of $(\alpha 7)_{2}$ into a planar lipid bilayer and a unilamellar liposome. (B) TEM image of $(a 7)_{2}$ inserted into two liposomes simultaneously (white arrows) and one liposome (yellow arrow). Scale bar, 20nm. 
A

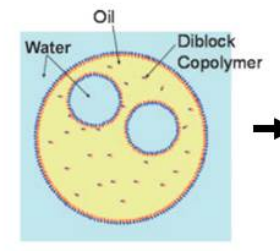

C

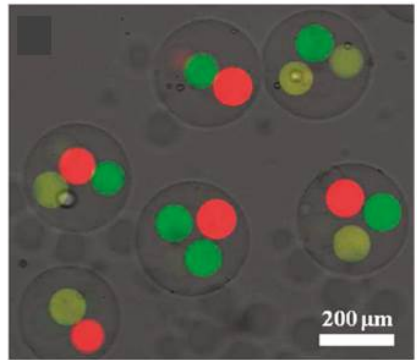

B

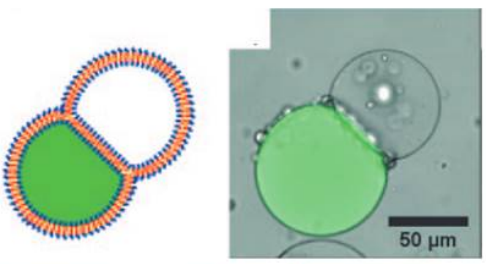

D
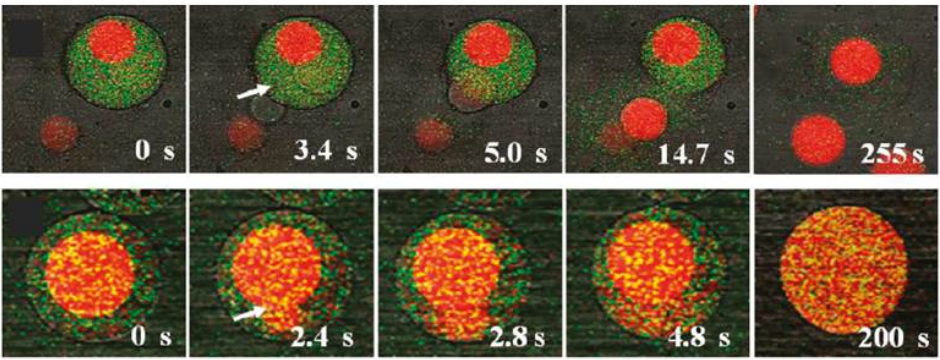

E

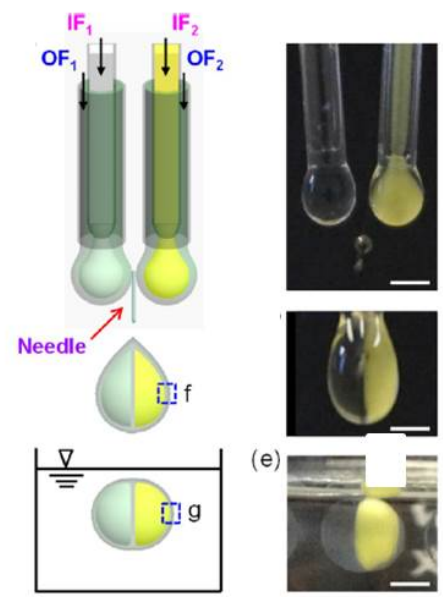

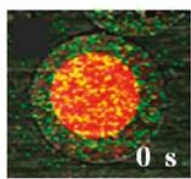
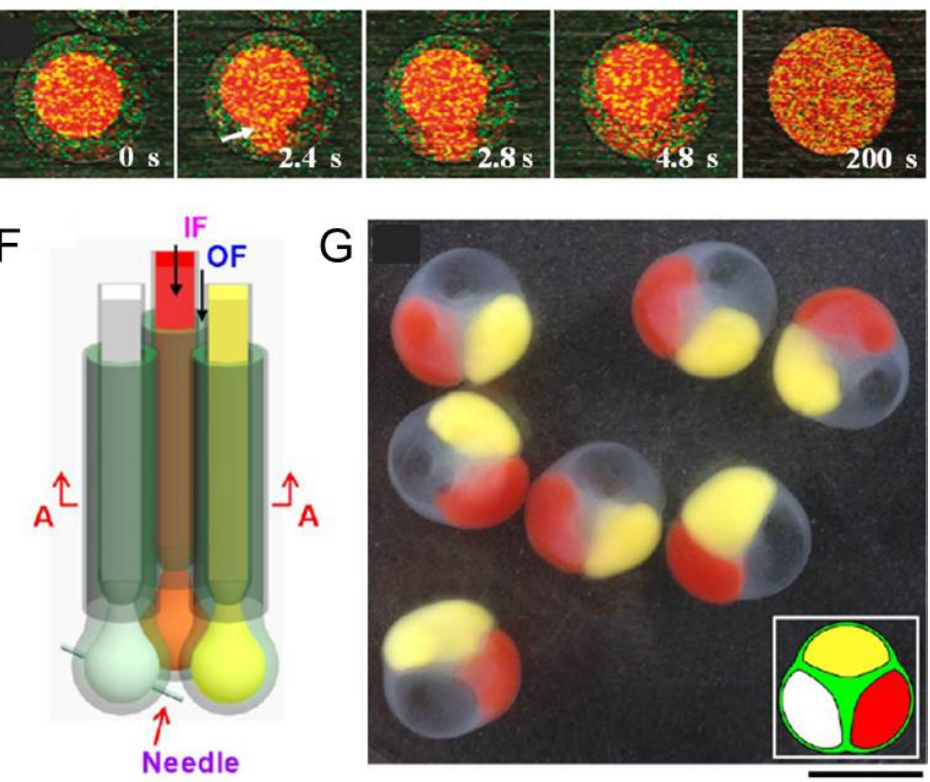

Figure 33. Structures with internal compartments. (A) Schematic of formation of multicompartment polymersomes from a w/o/w double emulsion with hexane/chloroform (yellow) as the oil phase. The interfaces are formed from an amphiphilic diblock copolymer, which is less soluble in hexane than chloroform. As the chloroform evaporates, dewetting and the expulsion of an oil droplet occurs. (B) Schematic and overlay of fluorescence and brightfield images of a two-compartment polymersome containing FITC-Dextran in one compartment ${ }^{174}$. (C) Confocal image of multi-compartment polymersomes, containing a red dye (sulforhodamine B), green dye (8-hydroxyl-1,3,6pyrenetrisulfonic acid trisodium salt), or a mixture of both red and green dye (yellow). (D) Concentric multilayer polymersomes. The outer (top panel) or inner (bottom panel) membranes can be selectively ruptured by tailoring their stabilities $^{175}$. (E) Schematic and photograph of the generation of 2compartment $\mathrm{Ca}^{2+}$-crosslinked alginate capsules by co-extrusion from a fluidic device. Scale bars, $2.5 \mathrm{~mm}$. (F) Generation of 3-compartment $\mathrm{Ca}^{2+}$ crosslinked alginate capsules. (G) 3-compartment capsules, with one compartment containing Disperse Red, one containing folic acid (yellow) and the other containing no dye ${ }^{180}$. Scale bar, $5 \mathrm{~mm}$. 


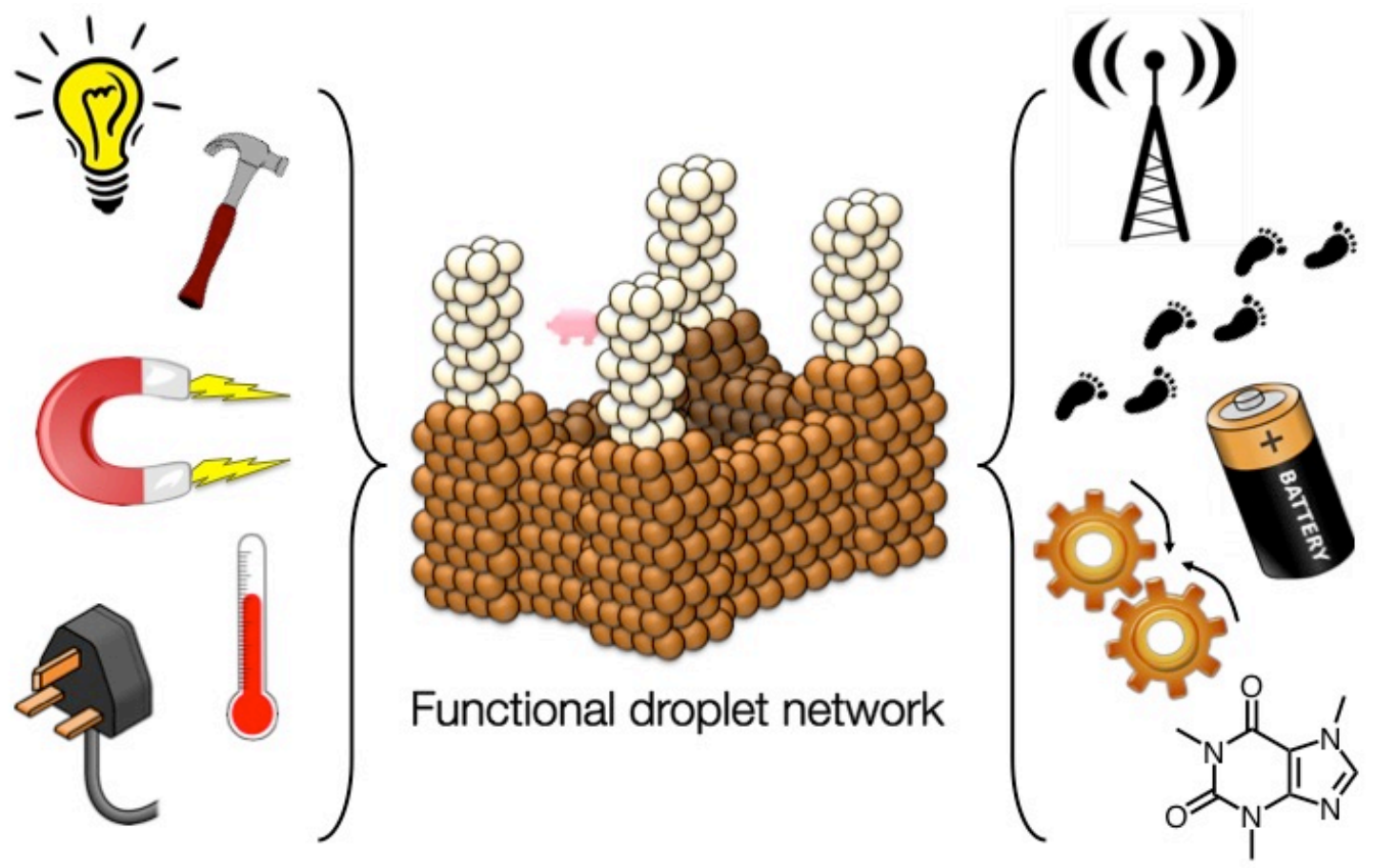

Figure 34. The future development of complex droplet networks, which respond to multiple stimuli and process them into functional outputs, will have many applications in biotechnology and medicine. Stimuli might include light, temperature, magnetism and mechanical or electrical forces. We envisage outputs to include external signalling, movement, soft battery power, and small molecule delivery. 